\title{
Combining Pd- and Chiral Organocatalysis for the Enantioselective Deconjugative Allylation of Enals via Dienamine Intermediates
}

\author{
Guang $\mathrm{Hu}$, Stacey E. Brenner-Moyer* \\ Department of Chemistry, Rutgers University, Newark \\ 73 Warren Street, Newark, NJ 07102, USA \\ seb244@rutgers.edu
}




\section{CONTENTS}

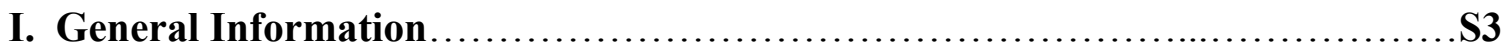

II . Reaction

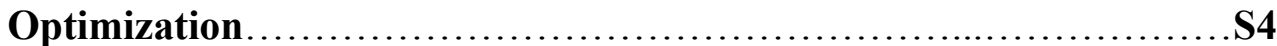

III. Determination of Double Bond Geometry in Cope Rearrangement Product...S9

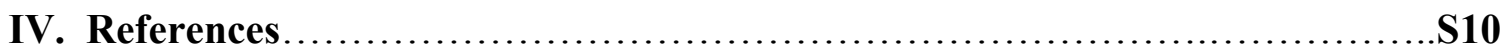

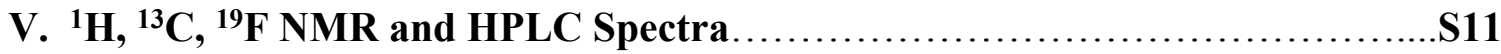




\section{I . General Information}

NMR data were acquired on Bruker $500 \mathrm{MHz}$ NMR spectrometers and use the following abbreviations: $\mathrm{s}=$ singlet, $\mathrm{d}=$ doublet, $\mathrm{t}=$ triplet, $\mathrm{m}=$ multiplet, $\mathrm{dd}=$ doublet of doublets, ddd $=$ doublet of doublets of doublets, $\mathrm{m}=$ multiplet, broad $\mathrm{s}=$ broad singlet. HRMS spectra were acquired using an MS spectrometer with Q-TOF mass analyzer. $[\alpha]_{\mathrm{D}}{ }^{22}$ were acquired using Jasco P-2000 Polarimeter. HPLC data were acquired on PerkinElmer Flexar HPLC set. Flash chromatography was carried out with 40-60 $\mu \mathrm{m}, 60 \AA$ silica gel and EMD silica 60 F254 glass TLC plates. Solvents were dried and kept air-free in a solvent purification unit, and were evaporated using a standard rotavapor and high vacuum. All reactions were carried out in oven-dried glassware, under an $\mathrm{Ar}$ atmosphere. 


\section{Reaction Optimization}

A number of other catalysts were evaluated to study their influence on regioselectivity. Guided by the mechanism proposed by List and Sunoj, ${ }^{1-2}$ in which approach of the Pd- $\pi$-allyl species is directed, in part, by a hydrogen bond from the enamine N-H to the phosphoric acid, we considered secondary amine catalysts, which would form enamines lacking an enamine $\mathrm{N}-\mathrm{H}$, but which incorporated pendant $\mathrm{N}-\mathrm{H}$ hydrogen bond donors at various tether lengths to direct allylation to the remote, $\gamma$-position. A bifunctional thiourea catalyst was ineffective in this reaction (entry 1, Table SI-1A). ${ }^{3}$ A squaramide catalyst proposed to direct remote functionalizations through $\mathrm{H}$-bond donation was next examined (entries 2-4). ${ }^{4}$ In contrast with List's conditions, which utilized an achiral amine and in which stereocontrol arose entirely from the use of catalytic chiral phosphoric acid, the phosphoric acid cocatalyst had no influence on the stereochemical outcome under these conditions. In fact, catalytic phosphoric acid provided slightly diminished enantio- and regioselectivities, albeit enhanced reactivities. Although this squaramide catalyst did generate the highest relative amount of $\gamma$-allylated product under these conditions, it still overwhelmingly favored allylation at the proximal, $\alpha$-position. To promote an expanded transition state, a squ-

Table SI-1A. Other Organocatalysts Evaluated.

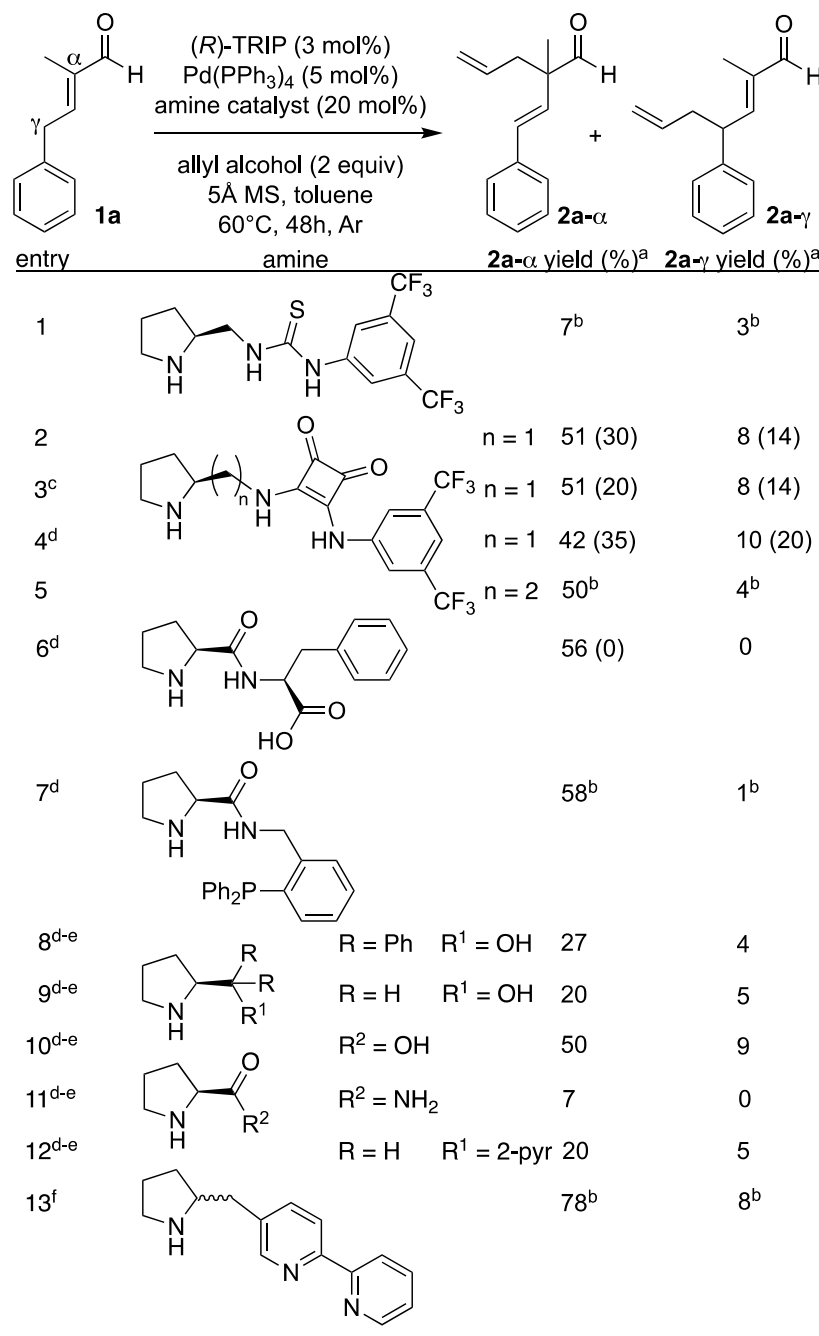

a Isolated yield. Number in parentheses is ee, which was determined by chiral phase HPLC ${ }^{b}{ }^{1} \mathrm{H}$ NMR yield using cyclohexene as internal standard. ${ }^{\mathrm{c}}(S)$-TRIP was used. ${ }^{\mathrm{d}}$ No TRIP was used. ${ }^{\mathrm{e}} \beta$-methallyl alcohol was used. ${ }^{\mathrm{f}}(R)$-TRIP (20 mol \%) was used. aramide catalyst with a longer tether length was synthesized, but did not improve selectivity for the remote position (entry 5). By analogy, single $\alpha$-amino acids, in conjunction with $\mathrm{Pd}$, catalyze $\alpha$-allylation of aldehydes via enamine formation. ${ }^{5}$ A dipeptide catalyst to enable an expanded transition state was, therefore, assessed, but afforded exclusively $\alpha$-allylated product (entry 6). Likewise, a catalyst with a tethered triaryl phosphine was examined. This catalyst provided low ee's in $\alpha$-allylation of cyclohexanone, ${ }^{6}$ but it was deemed possible that the tethered phosphine might be better situated for allylation at more remote positions. This catalyst too, however, favored allylation at the $\alpha$ position (entry 7). Similarly, catalysts with an appended alcohol, primary amide, or pyridine ${ }^{7}$ as other metal-coordinating groups did not produce a reversal in regioselectivity (entries 8-12). In addition to the homosquaramide catalyst, we also designed and synthesized a bifunctional bipyridine catalyst to chelate to, and remotely position, the metal center. This catalyst also failed to enhance the relative yield of $2 \mathbf{a}-\boldsymbol{\gamma}$ (entry 13).

Further primary and secondary amine catalysts were screened, but none significantly improved results. Primary amine catalysts that incorporated hydrogen bond donors were evaluated (entries 1-2, Table SI-1B), as were various other primary and secondary amine catalysts without pendant hydrogen bond donor groups (entries 3-8), but none provided improved results. Interestingly, however, as mentioned above, when a chiral secondary amine squaramide catalyst was used TRIP had little influence on enantioselectivity. The $\mathrm{m}$ ajor enantiomer of product was the same regardless of whether $(R)$ - or $(S)$-TRIP was used and the ee was slightly elevated when TRIP was altoge- 
ther absent (entries 2-4, Table S1-1A). When an achiral primary amine catalyst was used, however, the use of $(R)$ TRIP did afford some enantioselectivity (entry 7, Table SI-1B). This could possibly be explained by the List and counteranion model, in which there is a hydrogen bond from the enamine N-H to the chiral phosphoric acid, which, in turn, is coordinated to the Pd center. These interactions provide a close association of the proximal reactive center of the enamine with both the phosphoric acid and the reactive Pd species, enabling the relay of chiral information from the phosphoric acid during $\mathrm{C}-\mathrm{C}$ bond formation. The chiral phosphoric acid is evidently not so intimately involved in directing $\alpha$-allylation when secondary amine catalysts are used, possibly because the latter form enamines lacking an enamine $\mathrm{N}-\mathrm{H}$ bond.

Table SI-1B. Additional Amine Catalysts Evaluation.

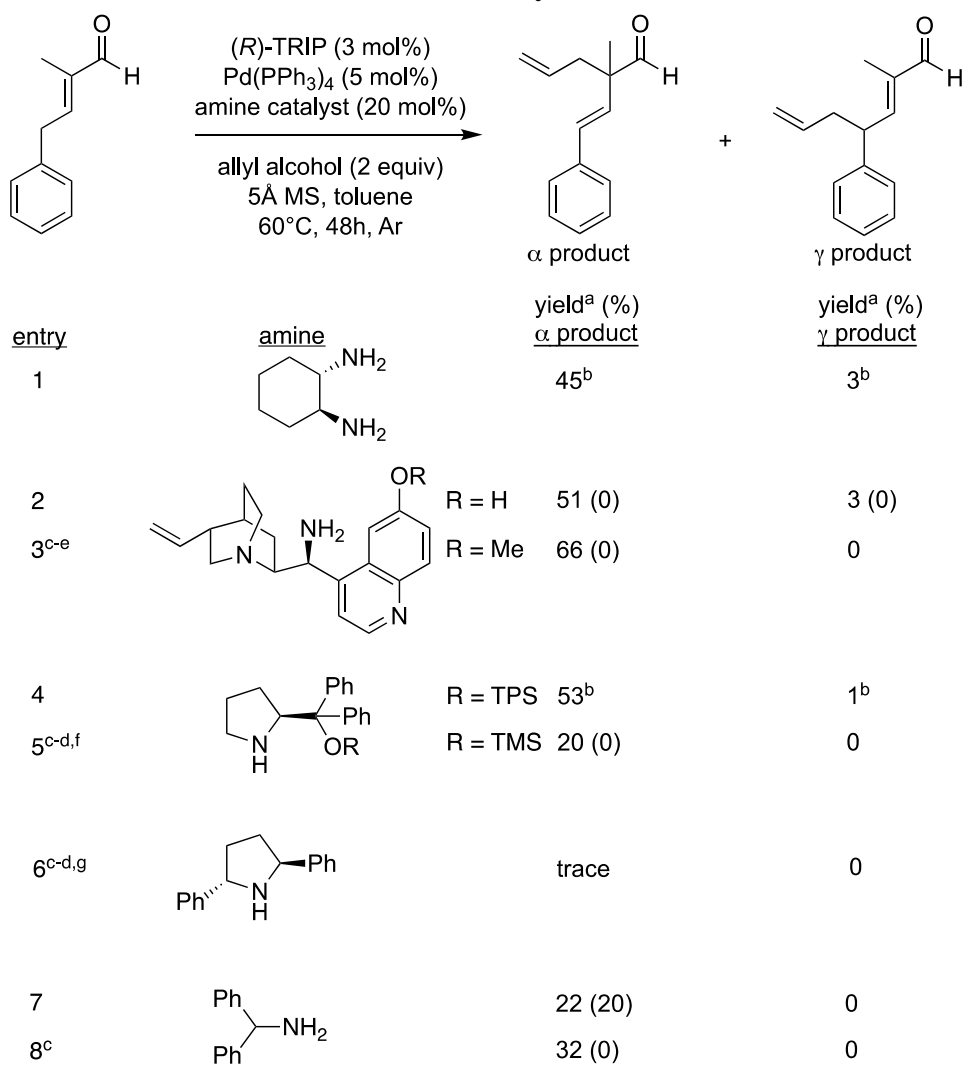

a Isolated yield unless otherwise noted. Number in parentheses is ee, which was determined by chiral phase HPLC. ${ }^{\mathrm{b}}$ NMR yield using cyclohexene as internal standard. ${ }^{\mathrm{c}}$ Allyl acetate (1-2 equiv) used instead of allyl alcohol. ${ }^{\mathrm{d}}$ No TRIP and no MS were used. ${ }^{\mathrm{e}}$ Reaction was run using amine catalyst $(10 \mathrm{~mol} \%)$ and $\mathrm{Pd}\left(\mathrm{PPh}_{3}\right)_{4}(30 \mathrm{~mol} \%)$ at rt. ${ }^{\mathrm{f}}$ Reaction was run in DMF/DMSO (1:1) at $-20{ }^{\circ} \mathrm{C}$. ${ }^{\mathrm{g}}$ Reaction was run using amine catalyst $\left(40 \mathrm{~mol} \%\right.$ ) for $1 \mathrm{~d}$ at $\mathrm{rt}$ and $1 \mathrm{~d}$ at $50{ }^{\circ} \mathrm{C}$.

As discussed above, the squaramide catalyst provided the highest relative amount of $\gamma$-allylated product when allyl alcohols were used. Using this catalyst, the effect of lower temperatures was investigated to possibly further enhance $\gamma$-allylation (Table SI-2). At room temperature, this reaction was completely shut down (entry 1). Switching to allyl acetate as the allyl source enabled the reaction to proceed at room temperature. Unfortunately, however, not only did the squaramide catalyst do nothing to improve selectivity for $\gamma$-position, it significantly hampered overall reactivity (entries 2-5). Although room temperature shut down the reaction using allyl alcohol, temperatures lower than $60{ }^{\circ} \mathrm{C}$ were tolerated. While the squaramide catalyst had a noticeable impact on regioselectivity under these conditions ( $>25: 1 \alpha: \gamma$ without catalyst, $\sim 5: 1 \alpha: \gamma$ with squaramide catalyst), with little to no impact on overall reactivity, there was still no significant improvement in the yield of the $\gamma$-allylated product (entries 6-9). 
Table SI-2. Additional investigations with squaramide catalyst.

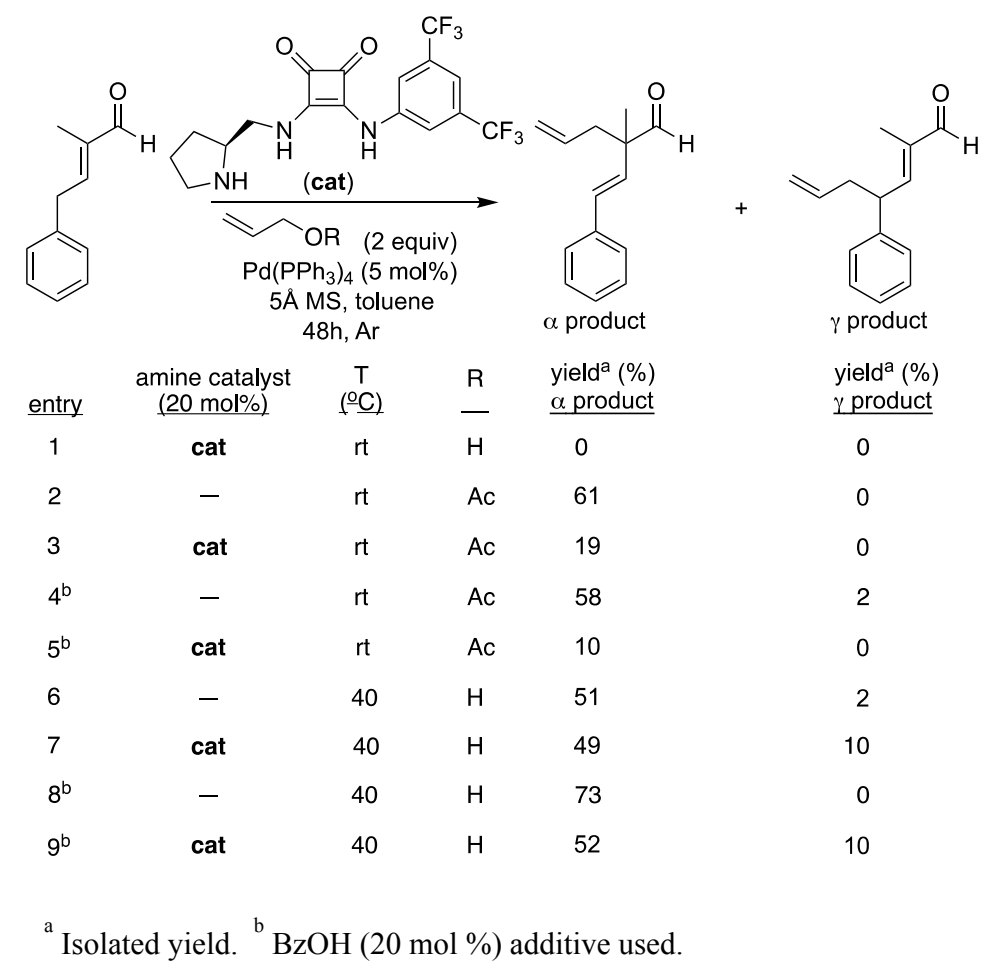

As mentioned, a bifunctional bipyridine catalyst was synthesized that was designed to chelate to the metal center to position it for remote allylation of enal substrates. In addition to the conditions reported in entry 13 of Table SI-1A, this novel catalyst was further examined under a variety of reaction conditions (Table SI-3). Unfortunately, neither changing the acid additive nor the Pd source was successful at reversing the regioselectivity of allylation (entries 1-4). Additionally, even the use of sterically bulky $\beta$-methallyl alcohol still favored formation of the $\alpha$-product (entries 5-6).

Table SI-3. Other optimizations with novel bifunctional bipyridine catalyst.<smiles>CC(C=O)=CCc1ccccc1</smiles>

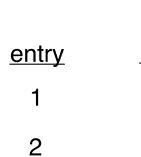

$2 \quad \mathrm{BzOH} \quad \mathrm{Pd}\left(\mathrm{PPh}_{3}\right)_{4} \quad \mathrm{H}$

$3 \quad(S)$-TRIP $\quad \mathrm{Pd}\left(\mathrm{PPh}_{3}\right)_{4} \quad \mathrm{H}$

$4 \quad-\quad \mathrm{Pd}(\mathrm{OAc})_{2} \quad \mathrm{H}$

$5 \quad-\quad \mathrm{Pd}\left(\mathrm{PPh}_{3}\right)_{4} \quad \mathrm{Me}$

$6 \quad-$

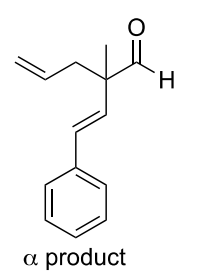

$$
\text { yield }^{\mathrm{a}}(\%)
$$$$
\underline{\alpha \text { product }}
$$$$
56
$$$$
75
$$$$
81
$$$$
0
$$$$
15
$$$$
0
$$<smiles>C=CCC(/C=C/C)c1ccccc1</smiles>

yield $^{\mathrm{a}}(\%)$ $\gamma$ product

4

1

8

0

3

${ }^{\text {a }}$ NMR yield using cyclohexene as internal standard. 
As evidenced by entry 4 in Table SI-1A, this transformation can proceed without TRIP, but also in the absence of dienamine formation (entries 1-3, Table SI-4). The latter presumably involves a dienol intermediate, which this substrate is primed to form. Reinforcing the challenge of catalyst regiocontrol in reactions of dienamines, the most effective means of favoring $\gamma$-allylation was through use of a sterically bulky allyl alcohol (i.e., by substrate control). In contrast to what was observed with the bifunctional bipyridine catalyst above, the squaramide catalyst, in conjunction with $\beta$-methallyl alcohol, was the sole instance of a $\gamma: \alpha$ product ratio $>1$ under these conditions (entries 4-5). Whereas TRIP enhanced reactivity but diminished ee's in reactions employing allyl alcohol (entries 2 vs. 4, Table SI-1A), it modestly enhanced the yield and significantly increased the ee of $\alpha$-allylated product when $\beta$-methallyl alcohol was used. Allylation of the dienol(ate) species favors $\alpha$-allylation in absence of amine, and in the presence of a basic tertiary amine incapable of dienamine formation (entries 3 and 6). This suggests that the squaramide catalyst may be influencing the regiochemical outcome of this reaction via dienamine formation.

\section{Table SI-4. Informative Control Reactions.}

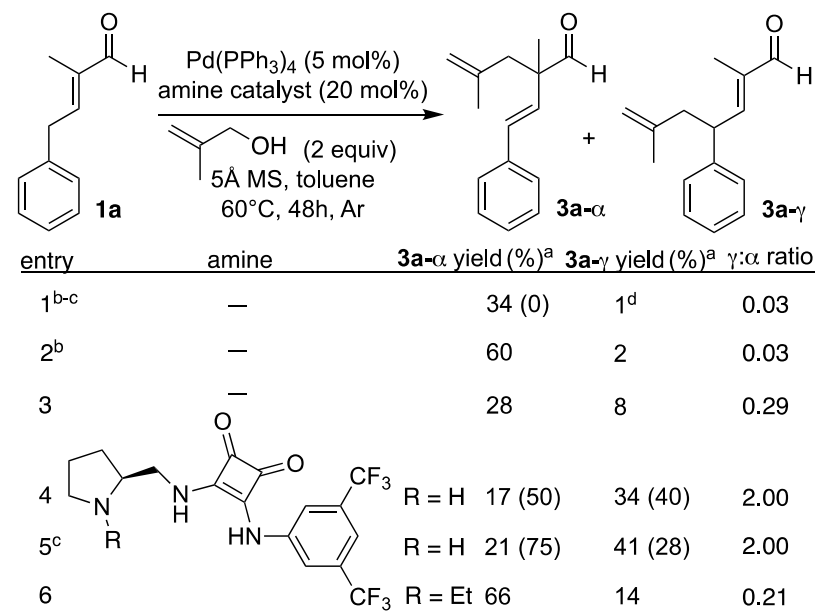

${ }^{a}$ Isolated yield. Number in parentheses is ee, which was determined by chiral phase HPLC. ${ }^{\mathrm{b}}$ Allyl alcohol was used. ${ }^{\mathrm{c}}(R)$-TRIP (3 mol \%) was used. ${ }^{\mathrm{d}}{ }^{1} \mathrm{H}$ NMR yield using cyclohexene as internal standard.

Cope rearrangement of the $\alpha$-product provided the $\gamma$-allylated enal. Temperatures under $110{ }^{\circ} \mathrm{C}$ were ineffective for this rearrangement, even in the absence of solvent (Table SI-5, entries 1-4). Higher dilution conditions did not enhance this intramolecular transformation (entries 5-6). The combination of 1,2dichlorobenzene as reaction solvent and a reaction temperature of $150{ }^{\circ} \mathrm{C}$ greatly improved the product yield (entry 7). Higher temperatures in this solvent did not further increase yields (entries 8-9).

Table SI-5. Preliminary optimizations of Cope rearrangement.

\begin{tabular}{|c|c|c|c|c|c|c|c|}
\hline & entry & $\begin{array}{l}\text { temp } \\
\left({ }^{\circ} \mathrm{C}\right)\end{array}$ & solvent & $\begin{array}{l}\text { conch } \\
\text { (M) }\end{array}$ & $\begin{array}{c}t \\
\text { (d) } \\
\end{array}$ & $\begin{array}{c}\text { yield } \\
(\%)\end{array}$ & \\
\hline & 1 & 80 & DCE & 0.5 & 6 & NR & \\
\hline & 2 & 80 & benzene & 0.5 & 5 & NR & \\
\hline & 3 & 100 & $O-D C B$ & 0.5 & 4 & $N R$ & \\
\hline & 4 & 100 & - & - & 5 & NR & \\
\hline & 5 & 110 & $\mathrm{PhMe}$ & 0.5 & 4 & 10 & \\
\hline & 6 & 110 & $\mathrm{PhMe}$ & 0.1 & 4 & 6 & \\
\hline & 7 & 150 & $o-\mathrm{DCB}$ & 0.5 & 2 & 45 & \\
\hline \multirow{2}{*}{ As mentioned in the text, } & 8 & 165 & $o-D C B$ & 0.5 & 2 & 40 & \multirow{2}{*}{ when the $\alpha$-Et substrate was } \\
\hline & 9 & 180 & $o$-DCB & 0.5 & 1 & 40 & \\
\hline
\end{tabular}


subjected to standard reaction conditions ( $1.5 \mathrm{~mol} \%(R)$-TRIP and $N$-allyl benzhydrylamine), the corresponding product was generated in $40 \%$ yield and $73 \%$ ee, with $40 \%$ of starting material remaining after $4 \mathrm{~d}$ (entry 1 , Table SI-6). Allyl amines that were less sterically congested led to comparable or slightly improved reactivities, but were detrimental to stereoselectivity (entries 2 and 4), while an allyl amine that restricted rotation of the two phenyl groups of the benzhydryl moiety failed to produce any product (entry 3). Similarly, chiral phosphoric acids containing 3,3'-bisphenyl groups with different substitution patterns than TRIP or without substitution, as well as those lacking 3,3'-bisphenyl groups entirely, resulted in racemic or nearly racemic products (entries 5-7). Changing the isopropyl groups on TRIP to less hindered Me groups significantly improved reactivity and stereoselectivity in this transformation (entry 8, Table SI-6; 2l, Scheme 1).

Table SI-6. Further optimizations with $\alpha$-Et substrate.

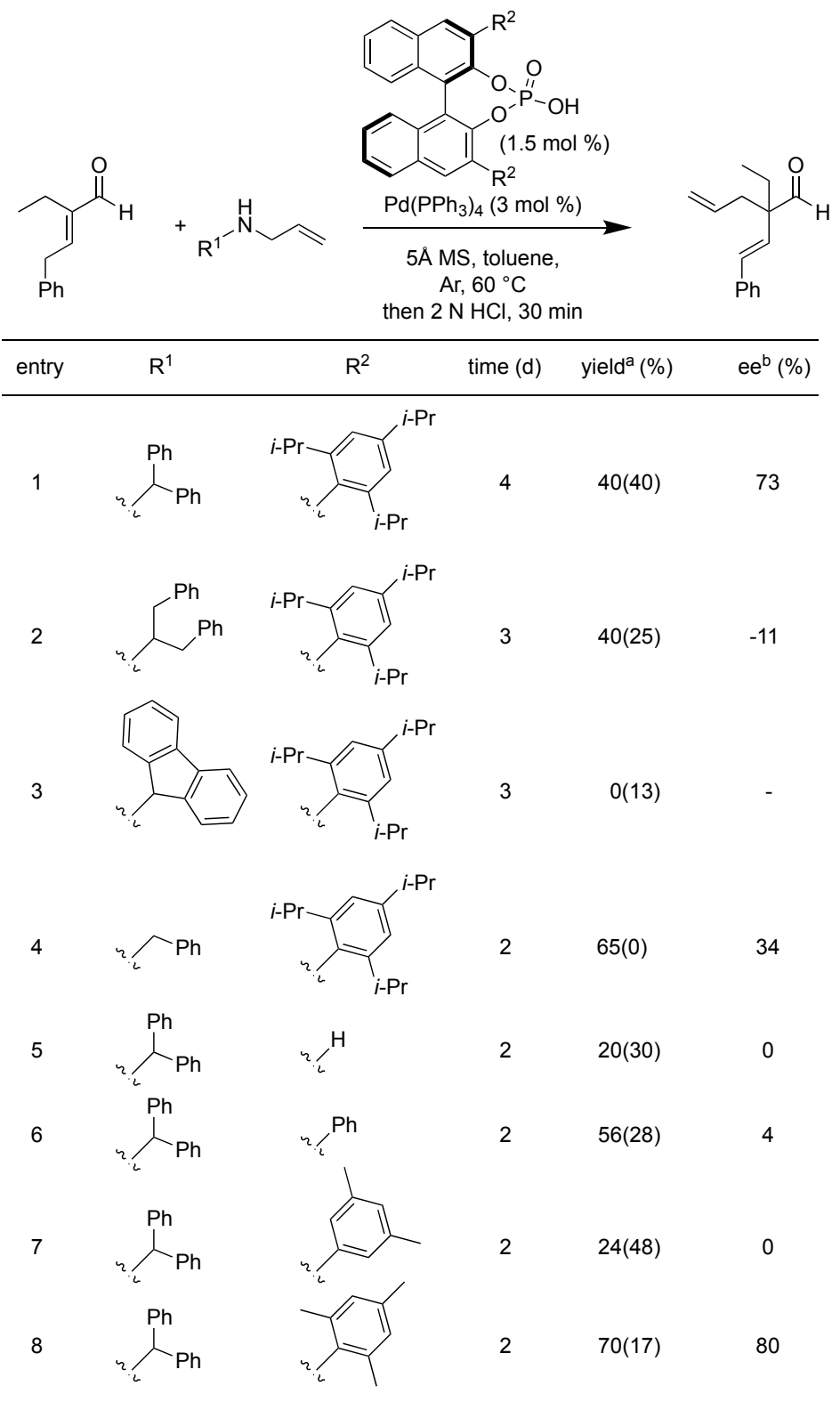

a Isolated yield of product; number in parenthesis is \% starting material remaining. ${ }^{b}$ ee determined by chiral phase HPLC of corresponding alcohol. 


\section{Determination of Double Bond Geometry in Cope Rearrangement Product}

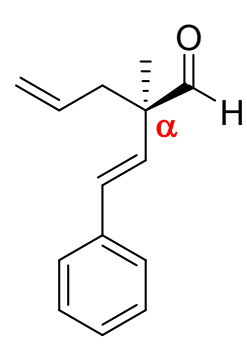

$96 \%$ ee

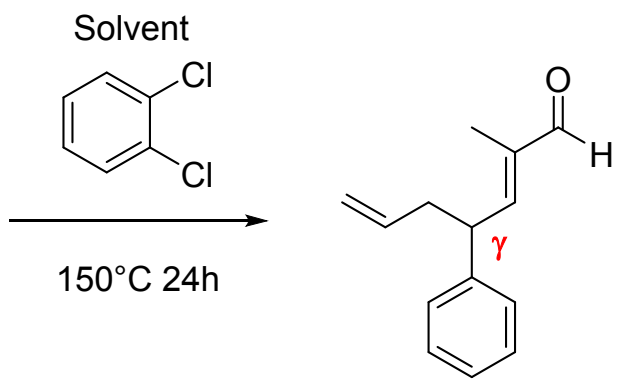

$45 \%$ yield

$93 \%$ ee

The E configuration of internal alkene in $\mathbf{2} \mathbf{a}-\boldsymbol{\gamma}$ is confirmed by NOE correlation between alkene- $\mathrm{H}$ and aldehyde- $\mathrm{H}$ is observed.

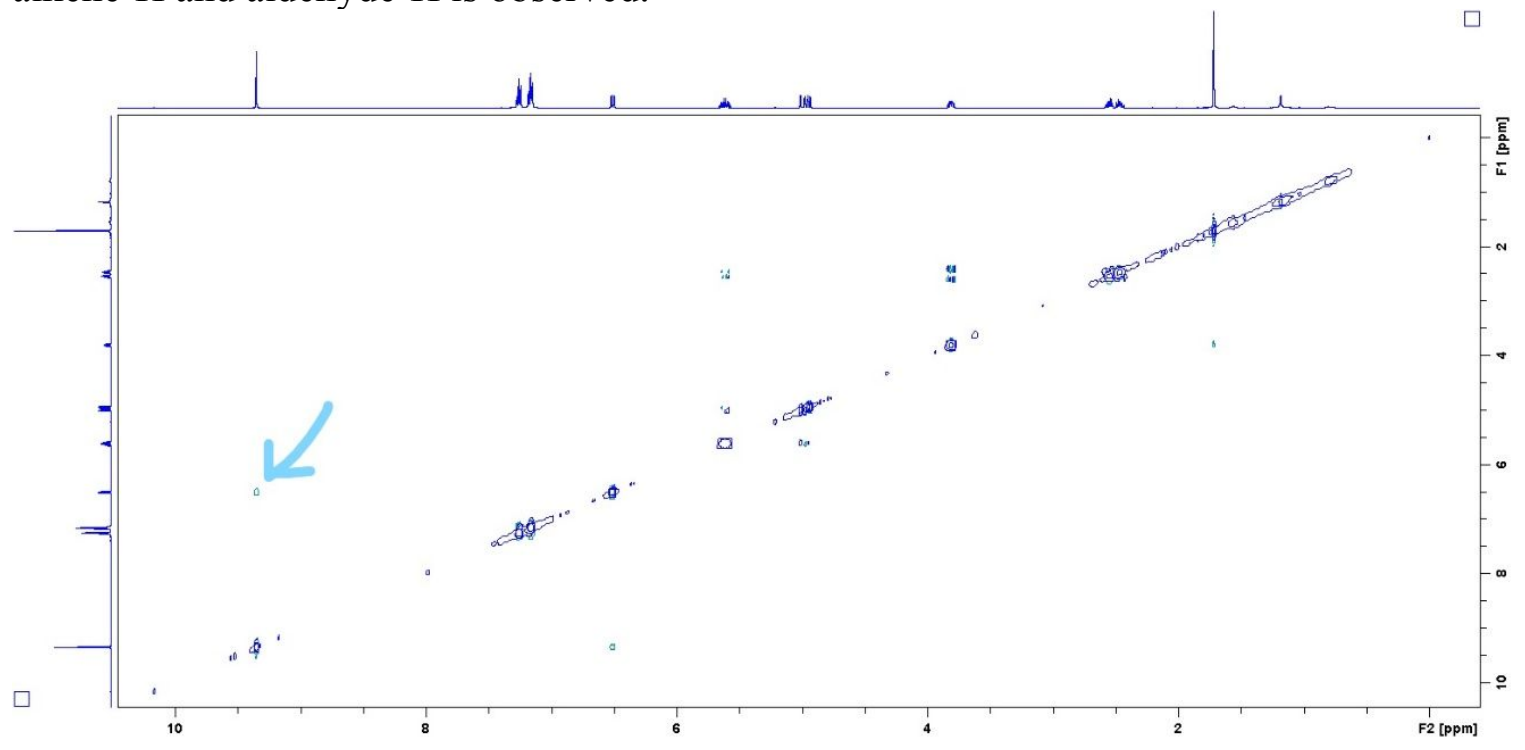




\section{References}

1. Mukherjee, S.; List, B. "Chiral counteranions in asymmetric transition-metal catalysis: highly enenatioselective $\mathrm{Pd} /$ Brønsted acid-catalyzed direct $\alpha$-allylation of aldehydes." J. Am. Chem. Soc. 2007, 129, 11336-11337.

2. Jindal, G.; Sunoj, R. B. "Mechanistic insights on cooperative asymmetric multicatalysis using chiral counterions." J. Org. Chem. 2014, 79, 7600-7606.

3. Cao, C.-L.; Ye, M.-C.; Sun, X.-L.; Tang, Y. "Enantioselective Michael addition of cyclohexanone to nitroolefins." Org. Lett. 2006, 8, 2901-2904.

4. a) Albrecht, L.; Dickmeiss, G.; Acosta, F. C.; Rodríguez-Escrich, C.; Davis, R. L.; Jørgensen, K. A. "Asymmetric organocatalytic formal [2+2]-cycloadditions via bifunctional H-bond directing dienamine catalysis." J. Am. Chem. Soc. 2012, 134, 2543-2546. b) Albrecht, L.; Acosta, F. C.; Fraile, A.; Albrecht, A.; Christensen, J.; Jørgensen, K. A. "Enantioselective H-bond-directing approach for trienaminemediated reactions in asymmetric synthesis." Angew. Chem Int. Ed. 2012, 51, 90889092. c) Albrecht, L.; Dickmeiss, G.; Weise, C. F.; Rodríguez-Escrich, C.; Jørgensen, K. A. "Dienamine-mediated inverse-electron-demand hetero-DielsAlder reaction by using an enantioselective H-bond-directing strategy." Angew. Chem Int. Ed. 2012, 51, 13109-13113. d) Albrecht, L.; Gómez, C. V.; Jacobsen, C. B.; Jørgensen, K. A. "1,4-Naphthoquinones in H-bond-directed trienaminemediated strategies." Org. Lett. 2013, 15, 3010-3013.

5. Yoshida, M.; Terumine, T.; Masaki, E.; Hara, S. "Direct asymmetric $\alpha$-allylation of $\alpha$-branched aldehydes by two catalytic systems with an achiral Pd complex and a chiral primary $\alpha$-amino acid." J. Org. Chem. 2013, 78, 10853-10859.

6. Yasuda, S.; Kumagai, N.; Shibasaki, M. "Direct asymmetric $\alpha$-allylation of ketones with allylic alcohols via Pd/enamine cooperative function." Heterocycles 2012, 86, 745-757.

7. Ishii, T.; Fujioka, S.; Sekiguchi, Y.; Kotsuki, H. "A new class of chiral pyrrolidine-pyridine conjugate base catalysts for use in asymmetric Michael addition reactions.” J. Am. Chem. Soc. 2004, 126, 9558-9559. 


\section{NMR and HPLC spectra}

1e: (E)-2-methyl-4-(4-(trifluoromethyl)phenyl)but-2-enal:

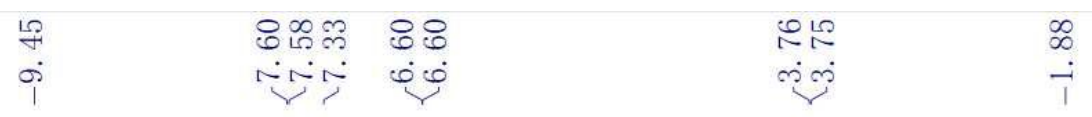

${ }^{1} \mathrm{H} \mathrm{NMR}\left(500 \mathrm{MHz}, \mathrm{CDCl}_{3}\right)$<smiles>C/C(C=O)=C\Cc1ccc(C(F)(F)F)cc1</smiles>

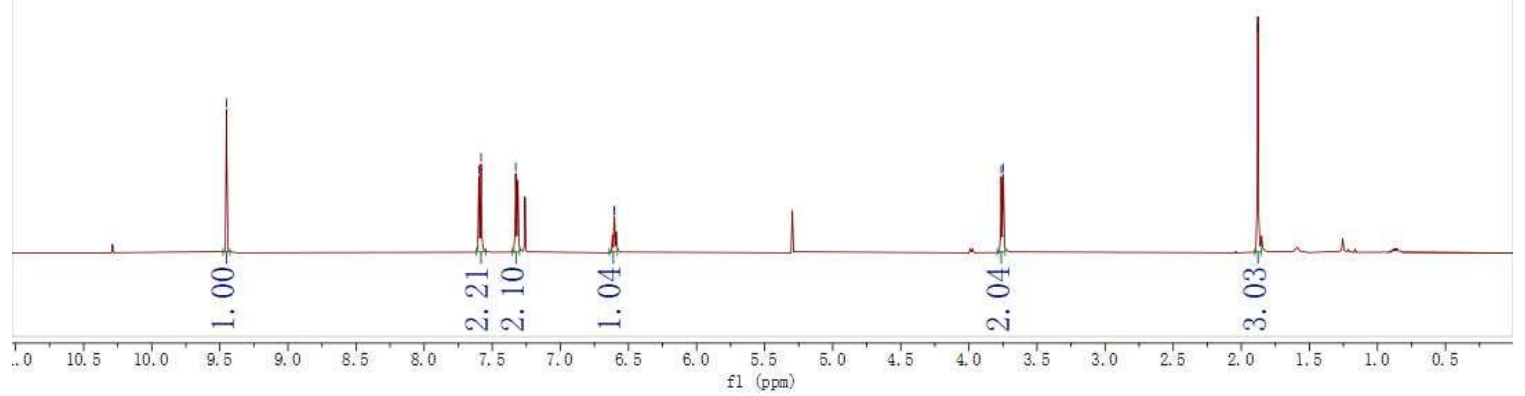


${ }^{19} \mathrm{~F}$ NMR (471 MHz, $\mathrm{CDCl}_{3}$ )

\section{온
ค่
1}<smiles>C/C(C=O)=C\Cc1ccc(C(F)(F)F)cc1</smiles>
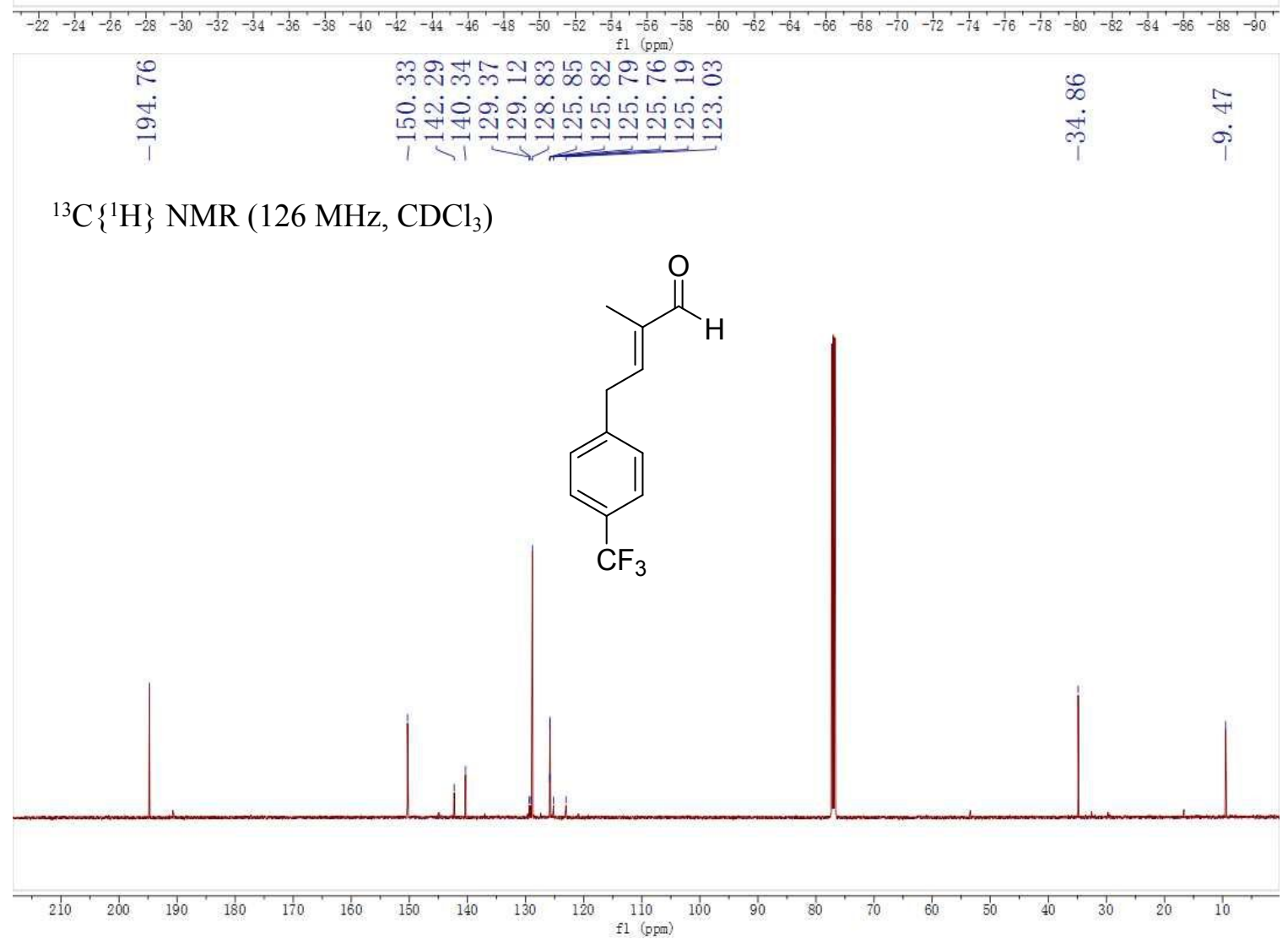
1f: (E)-4-(3-methyl-4-oxobut-2-en-1-yl)benzonitrile

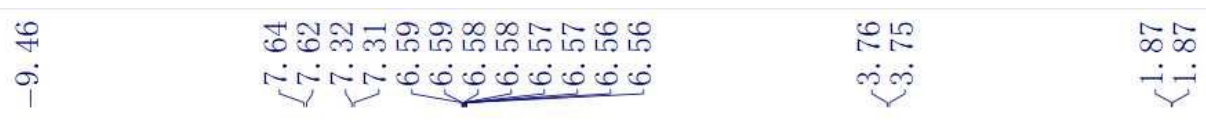

${ }^{1} \mathrm{H}$ NMR $\left(500 \mathrm{MHz}, \mathrm{CDCl}_{3}\right)$<smiles>C/C(C=O)=C\Cc1ccc(C#N)cc1</smiles>
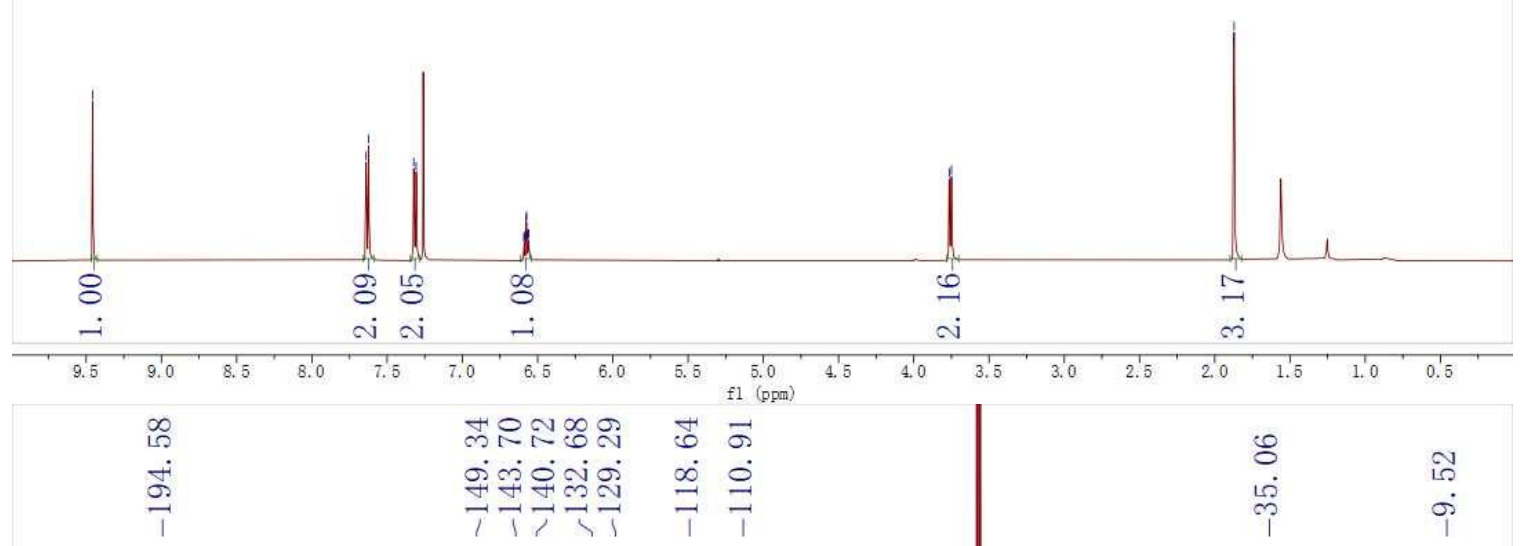

${ }^{13} \mathrm{C}\left\{{ }^{1} \mathrm{H}\right\} \mathrm{NMR}\left(126 \mathrm{MHz}, \mathrm{CDCl}_{3}\right)$<smiles>C/C(C=O)=C\Cc1ccc(C#N)cc1</smiles>

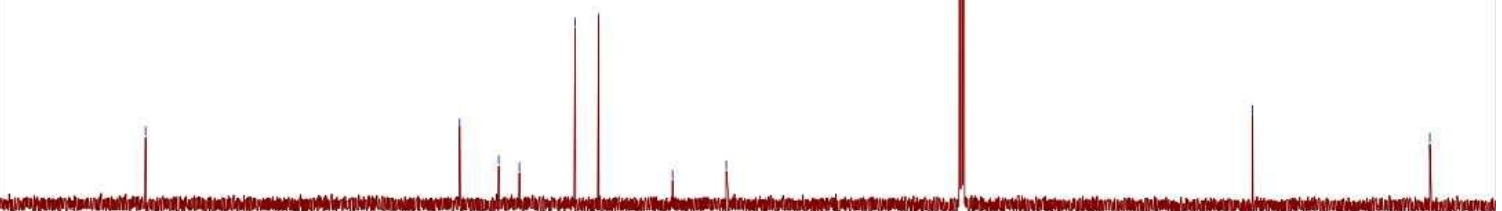

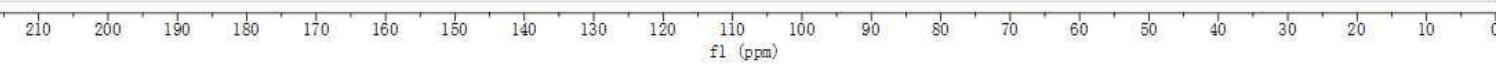


1h: (E)-2-methyl-4-(2-(trifluoromethyl)phenyl)but-2-enal

ๆ DNNNNNNNNNNN 0000000

${ }^{1} \mathrm{H}$ NMR $\left(500 \mathrm{MHz}, \mathrm{CDCl}_{3}\right)$

$\begin{array}{ll}\infty \infty & \infty \infty \\ \infty \infty \infty & \infty \\ \sim \infty & \sim-j\end{array}$

$-$

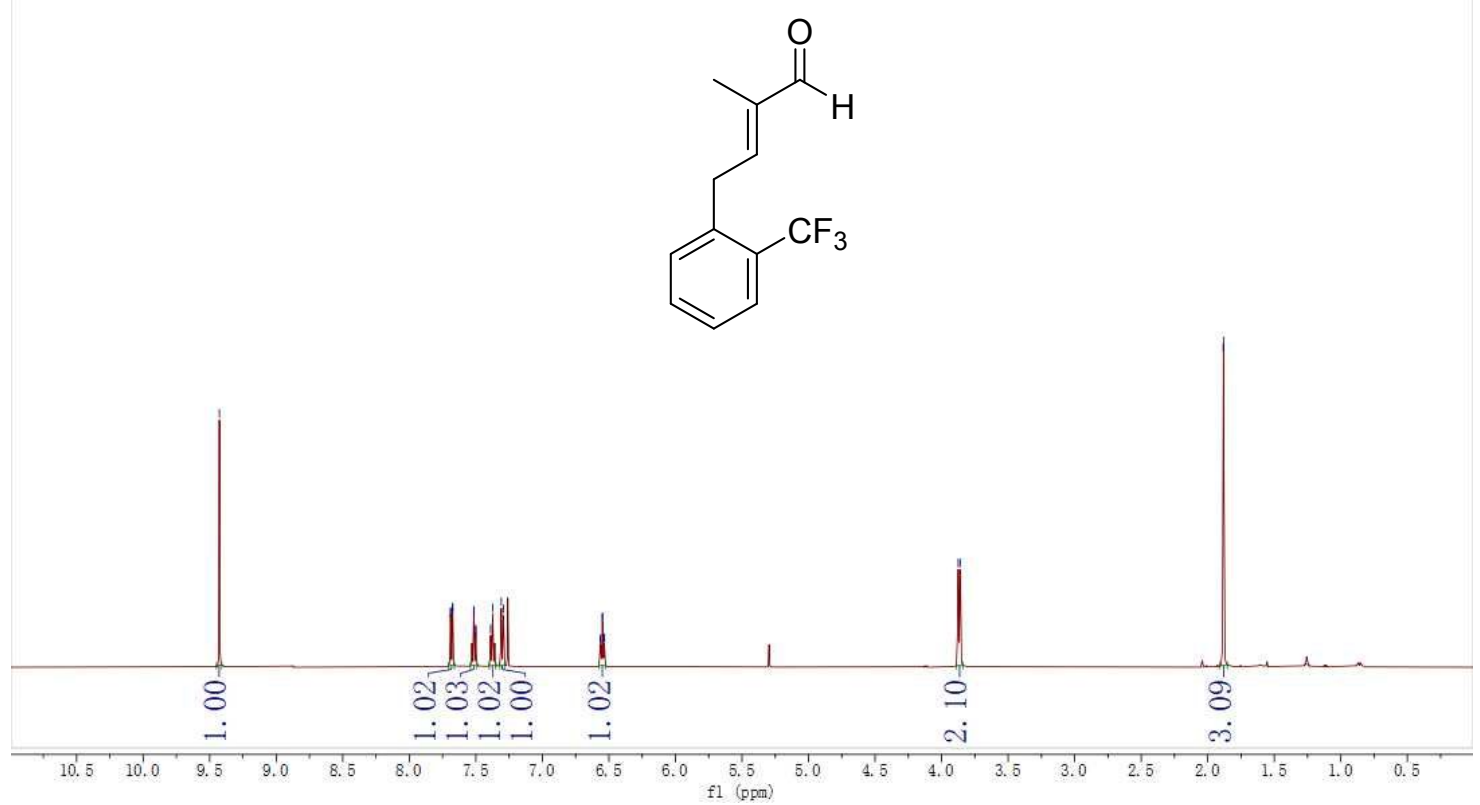


${ }^{19} \mathrm{~F}$ NMR (471 MHz, $\mathrm{CDCl}_{3}$ )<smiles>C/C(=C\Cc1ccccc1C(F)(F)F)C(F)(F)F</smiles>
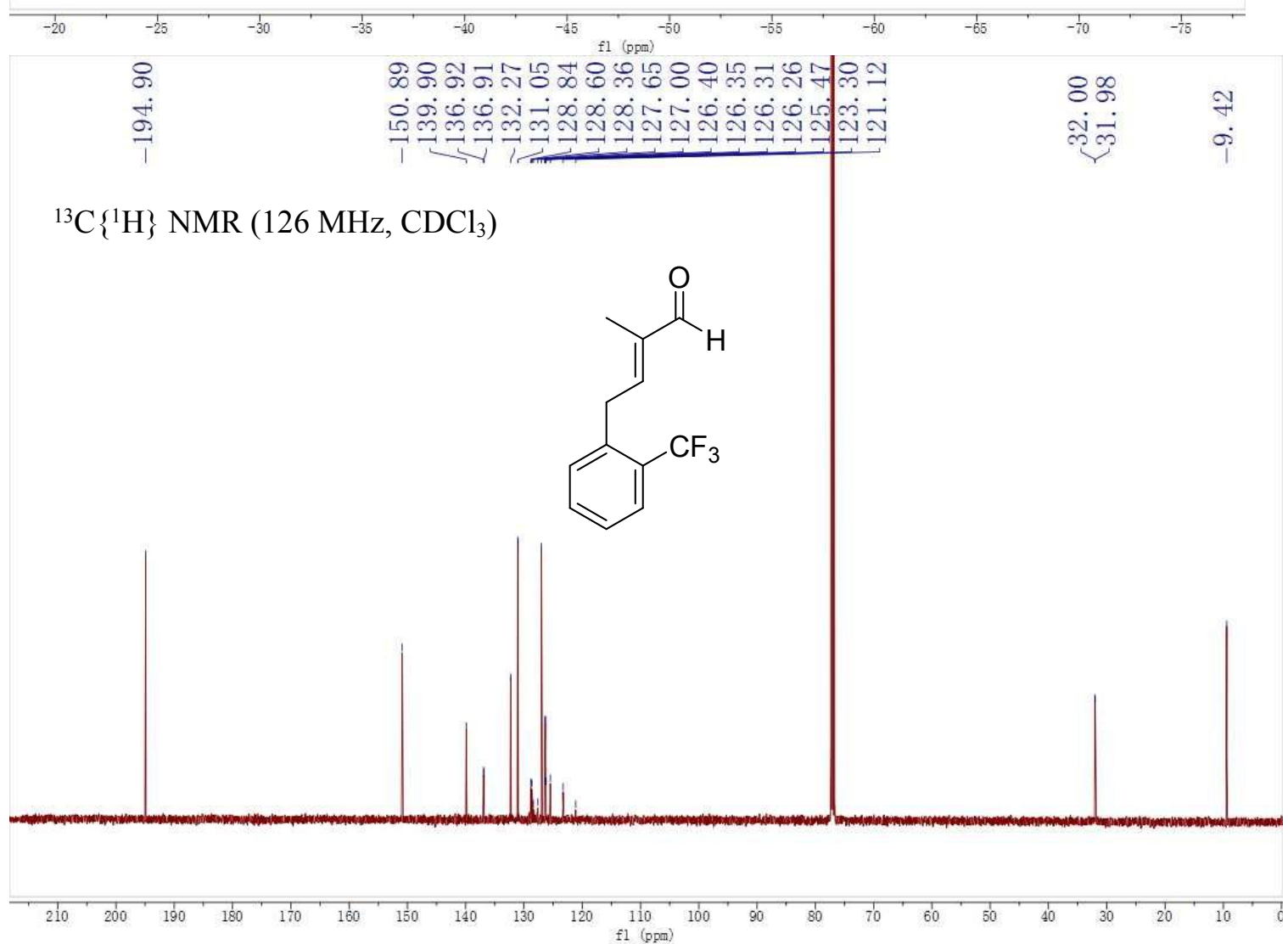
1j: 2-(3,4-dihydronaphthalen-2-yl)propanal
จึ
jं
고낭낭 $\infty$
Nin
ลีลฒ กิ กิก
भुंब रुं चं

${ }^{1} \mathrm{H}$ NMR $\left(500 \mathrm{MHz}, \mathrm{CDCl}_{3}\right)$<smiles>CC(C=O)C1=Cc2ccccc2CC1</smiles>
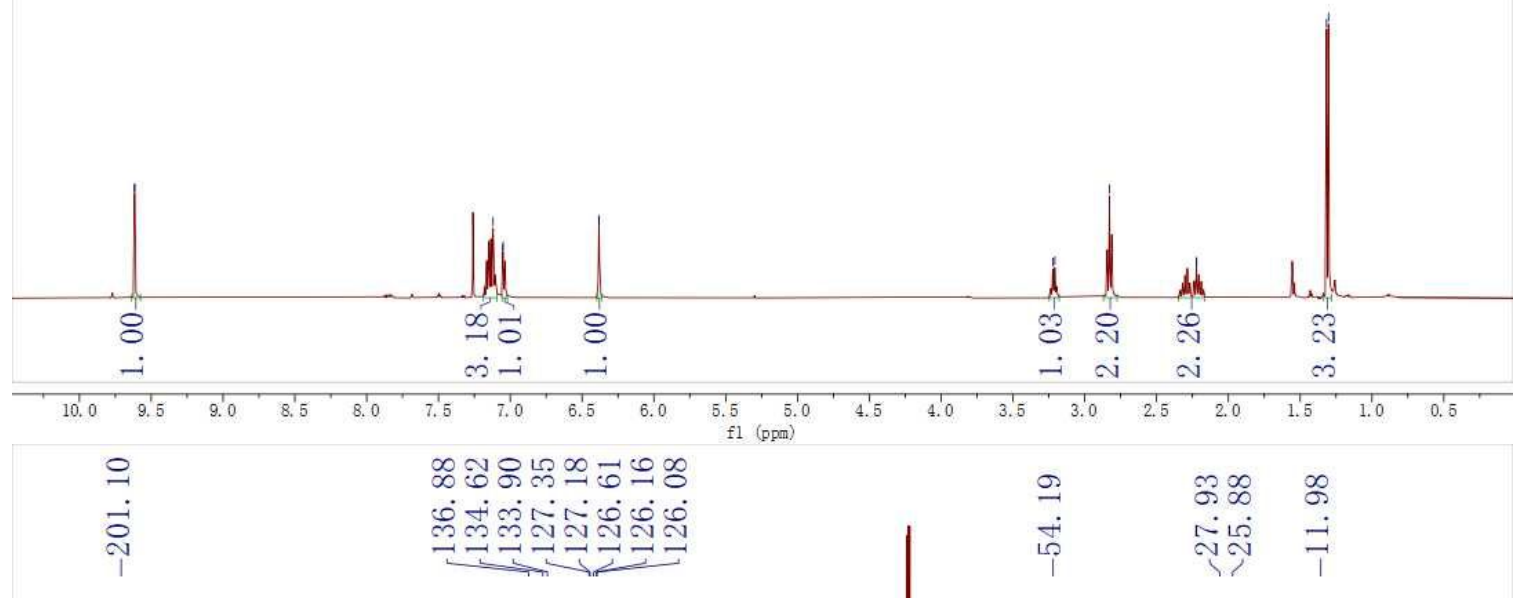

${ }^{13} \mathrm{C}\left\{{ }^{1} \mathrm{H}\right\} \mathrm{NMR}\left(126 \mathrm{MHz}, \mathrm{CDCl}_{3}\right)$<smiles>CC(C=O)C1=Cc2ccccc2CC1</smiles>

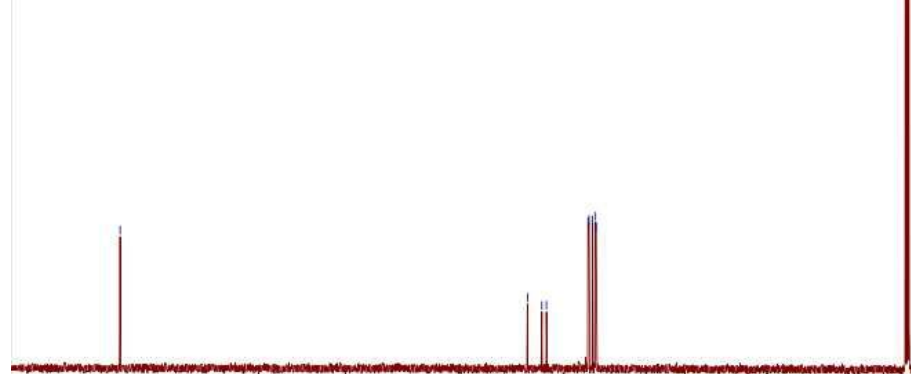

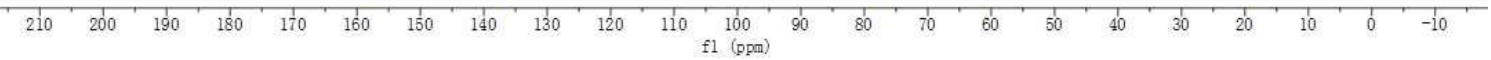


1k: tert-butyl (E)-2-(3-methyl-4-oxobut-2-en-1-yl)-1H-pyrrole-1-carboxylate

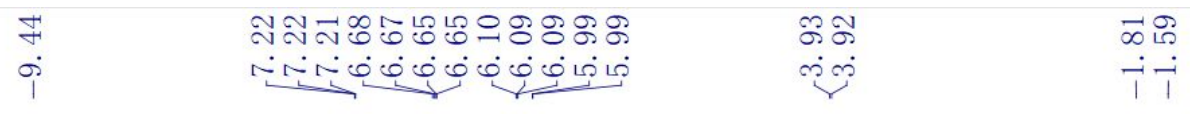

${ }^{1} \mathrm{H}$ NMR (500 MHz, $\mathrm{CDCl}_{3}$ )
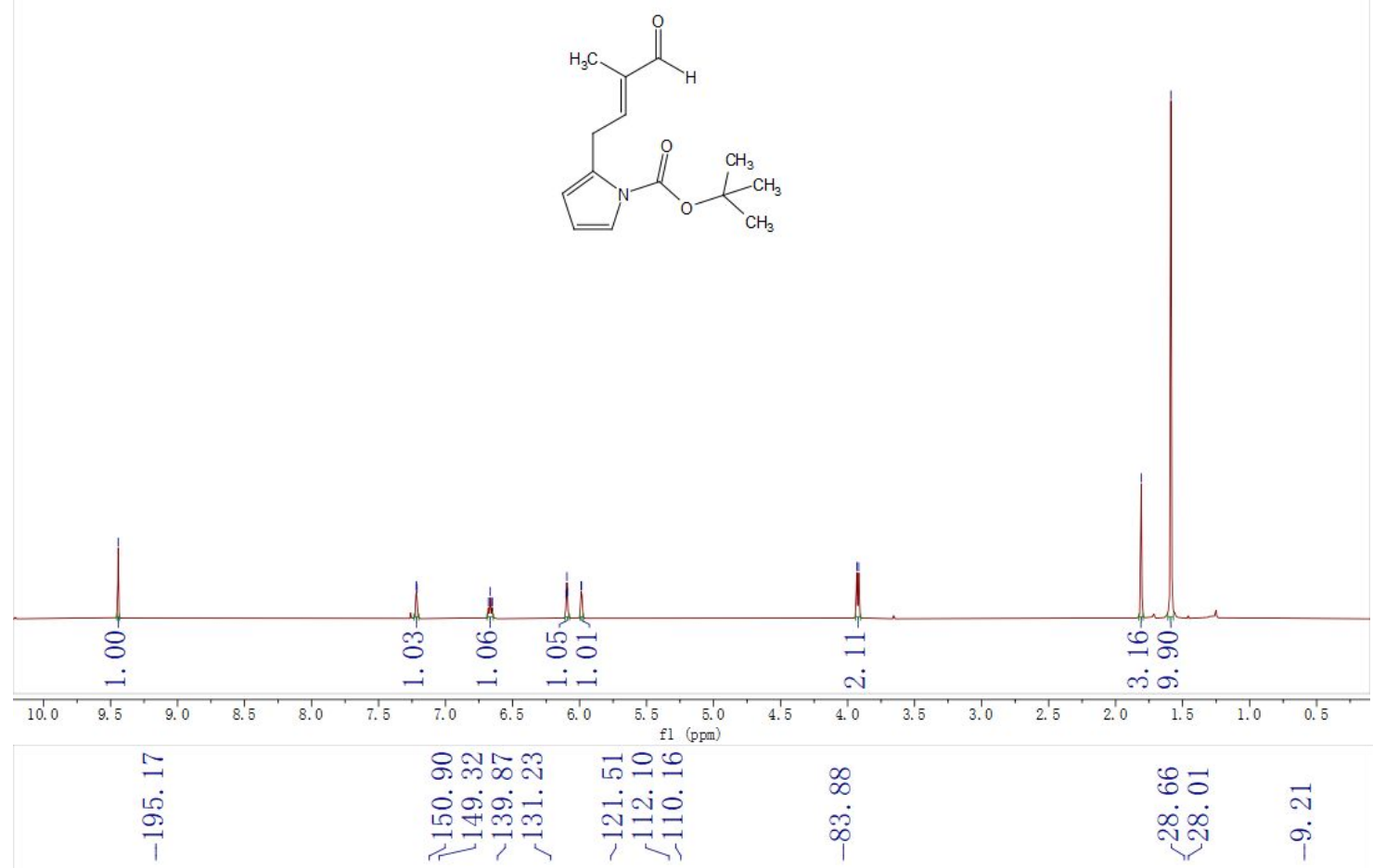

${ }^{13} \mathrm{C}\left\{{ }^{1} \mathrm{H}\right\} \mathrm{NMR}\left(126 \mathrm{MHz}, \mathrm{CDCl}_{3}\right)$
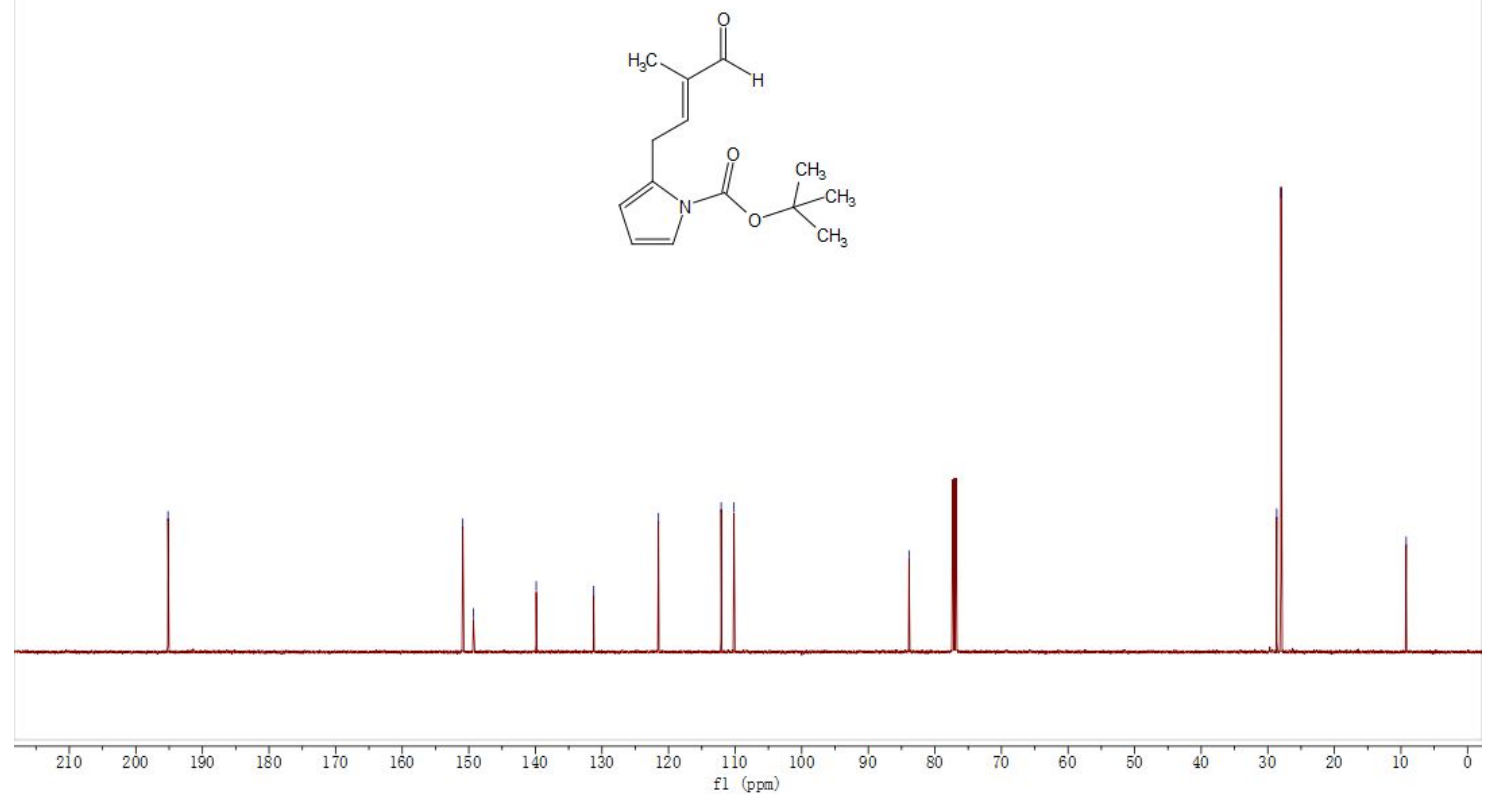
4a: N-benzhydryl-2-methylprop-2-en-1-amine

\begin{tabular}{|c|c|c|}
\hline 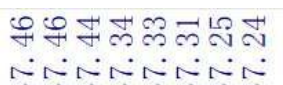 & 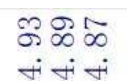 & $\stackrel{0}{\oplus}$ \\
\hline
\end{tabular}

${ }^{1} \mathrm{H}$ NMR $\left(500 \mathrm{MHz}, \mathrm{CDCl}_{3}\right)$

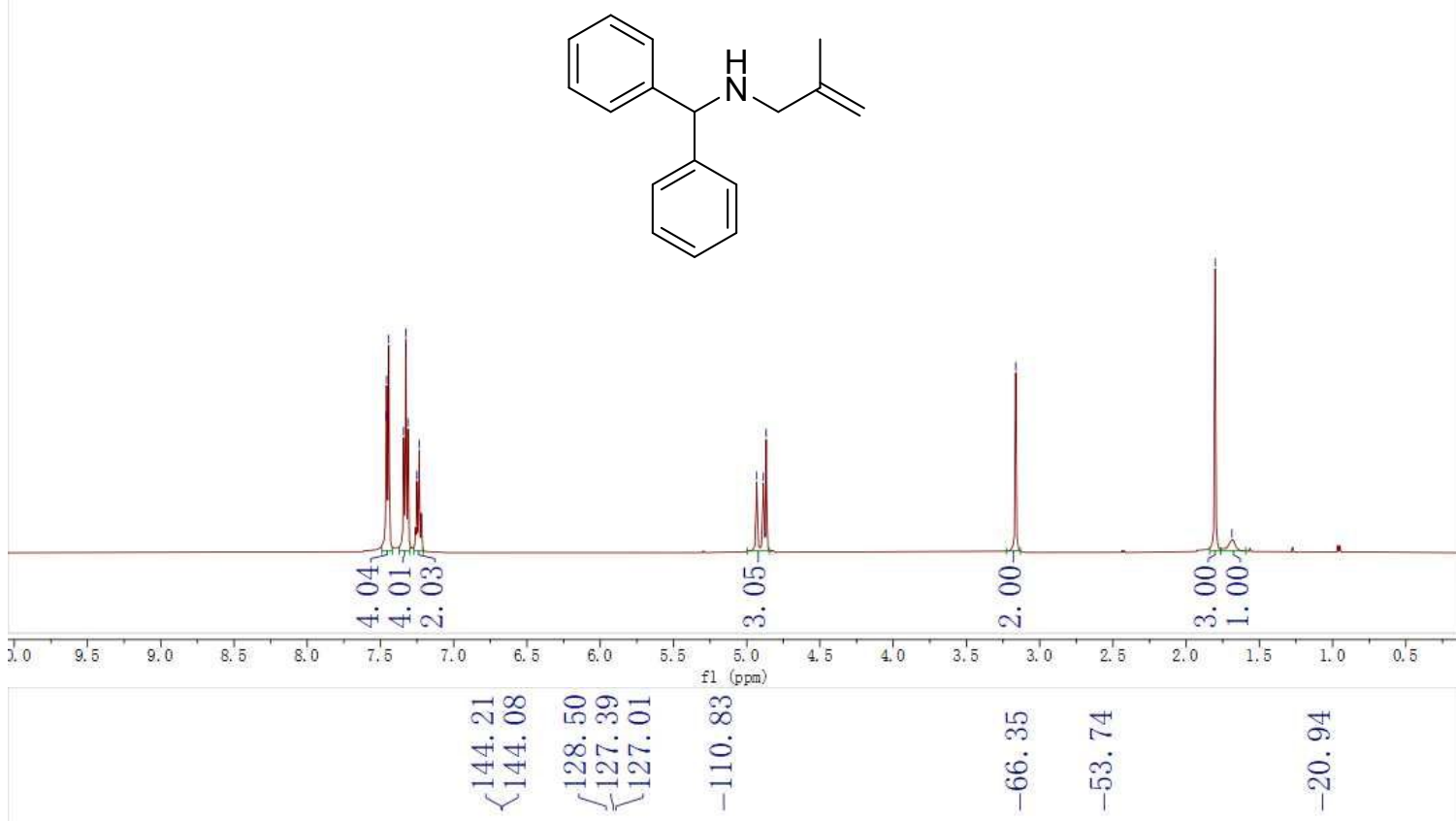

${ }^{13} \mathrm{C}\left\{{ }^{1} \mathrm{H}\right\} \mathrm{NMR}\left(126 \mathrm{MHz}, \mathrm{CDCl}_{3}\right)$
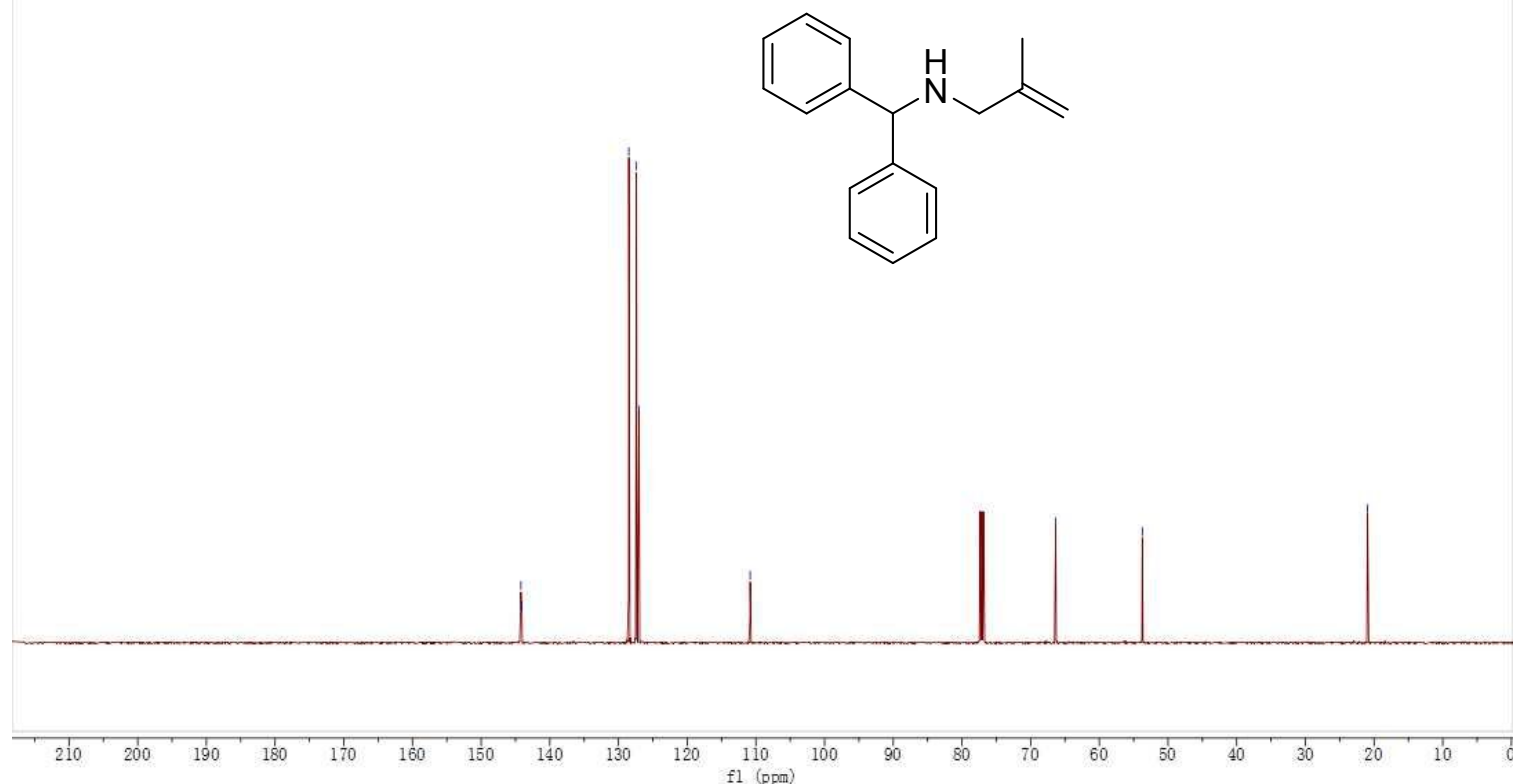
2a: $(S, E)$-2-methyl-2-styrylpent-4-enal

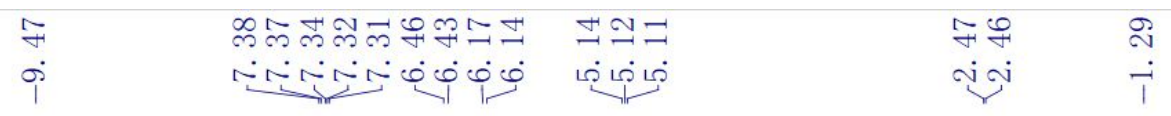

${ }^{1} \mathrm{H}$ NMR $\left(500 \mathrm{MHz}, \mathrm{CDCl}_{3}\right)$
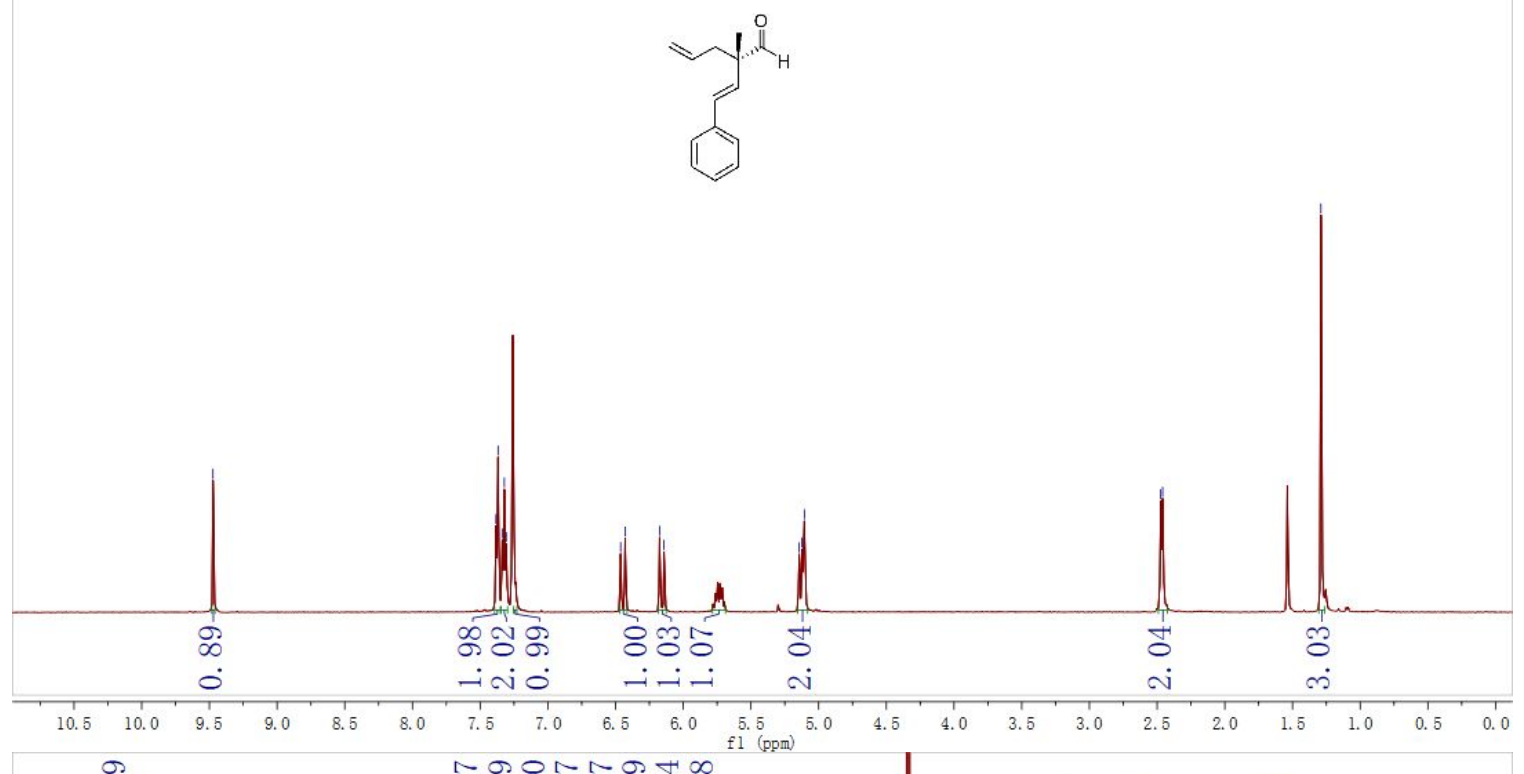

@

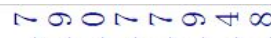

๑่

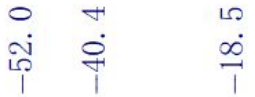

${ }^{13} \mathrm{C}\left\{{ }^{1} \mathrm{H}\right\}$ NMR $\left(126 \mathrm{MHz}, \mathrm{CDCl}_{3}\right)$
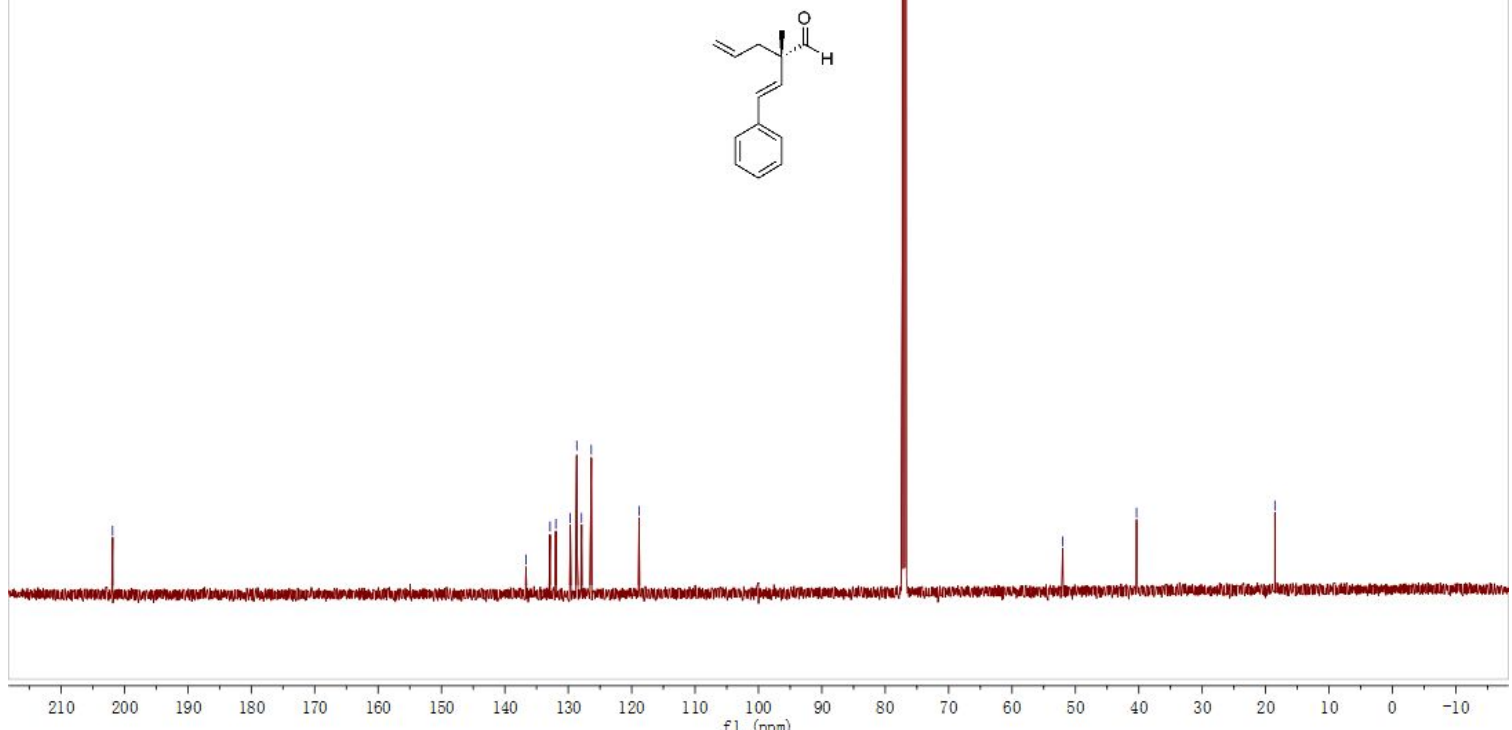

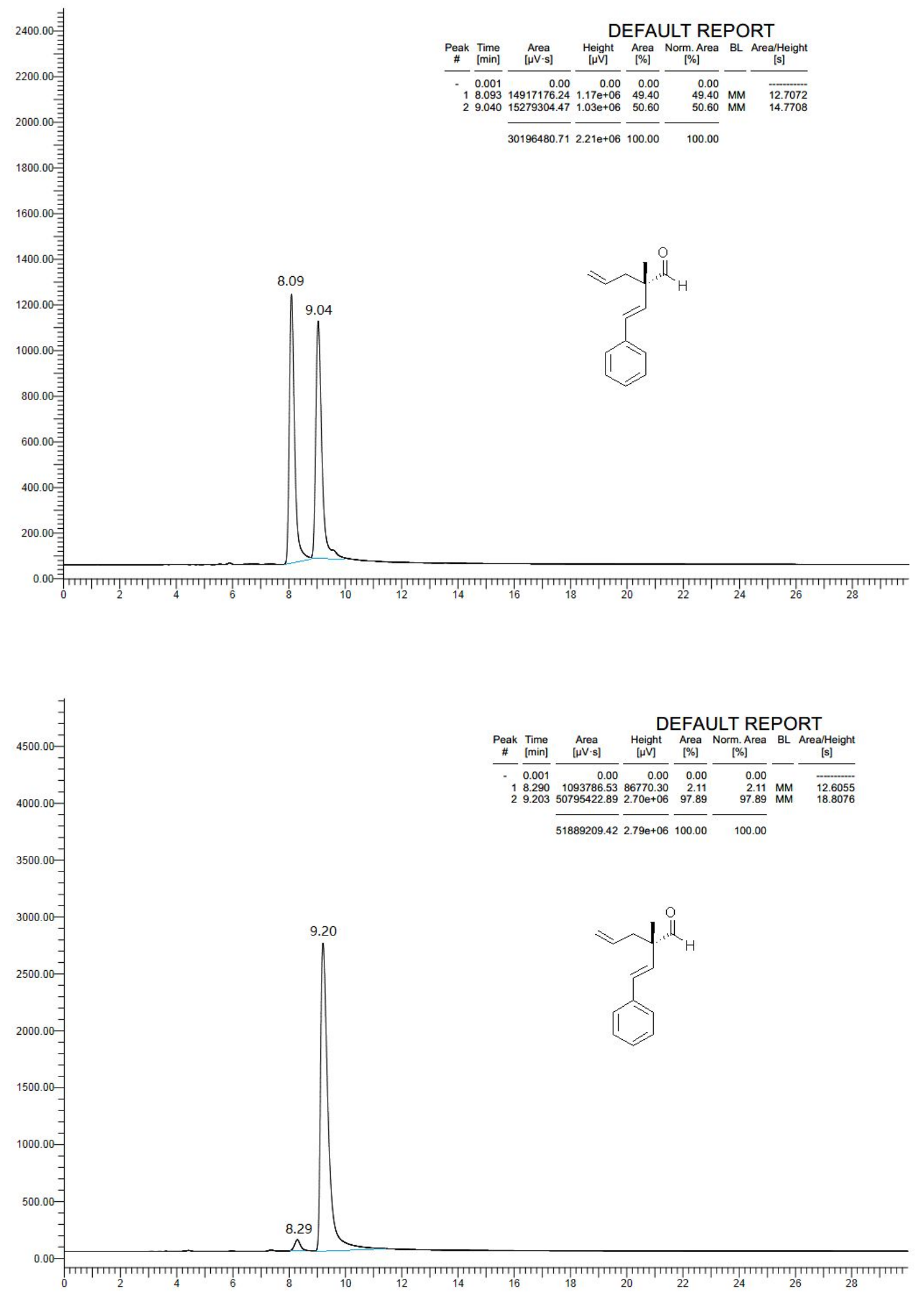
2b: (S,E)-2-(4-methoxystyryl)-2-methylpent-4-enal

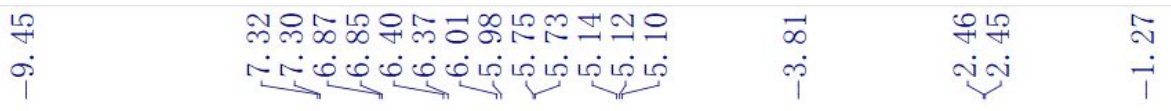

${ }^{1} \mathrm{H}$ NMR $\left(500 \mathrm{MHz}, \mathrm{CDCl}_{3}\right)$
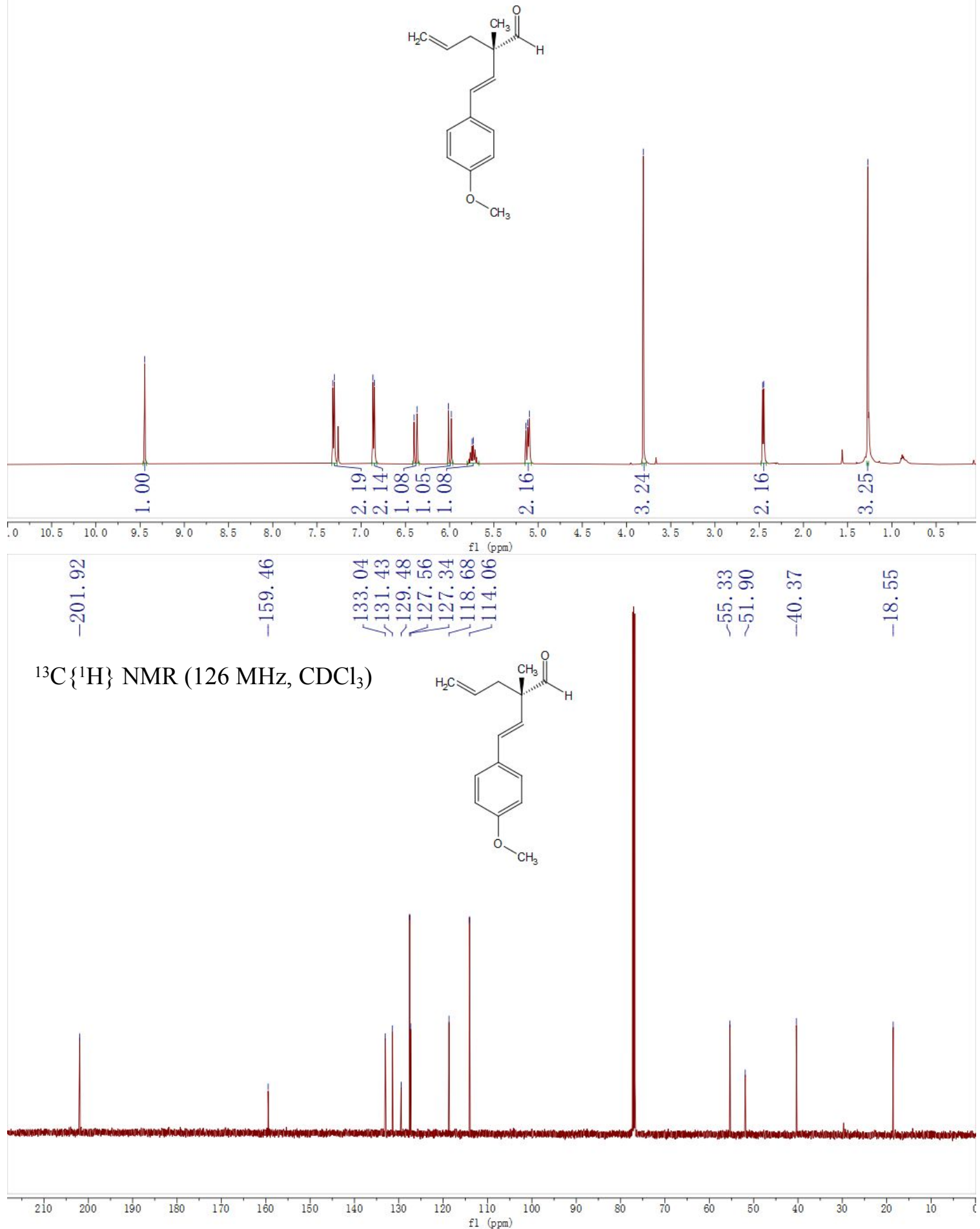


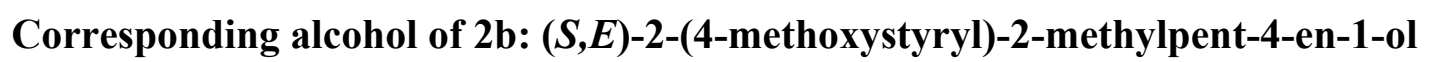

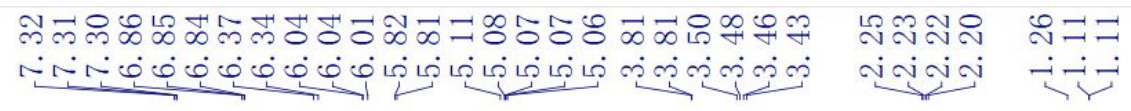

${ }^{1} \mathrm{H}$ NMR $\left(500 \mathrm{MHz}, \mathrm{CDCl}_{3}\right)$

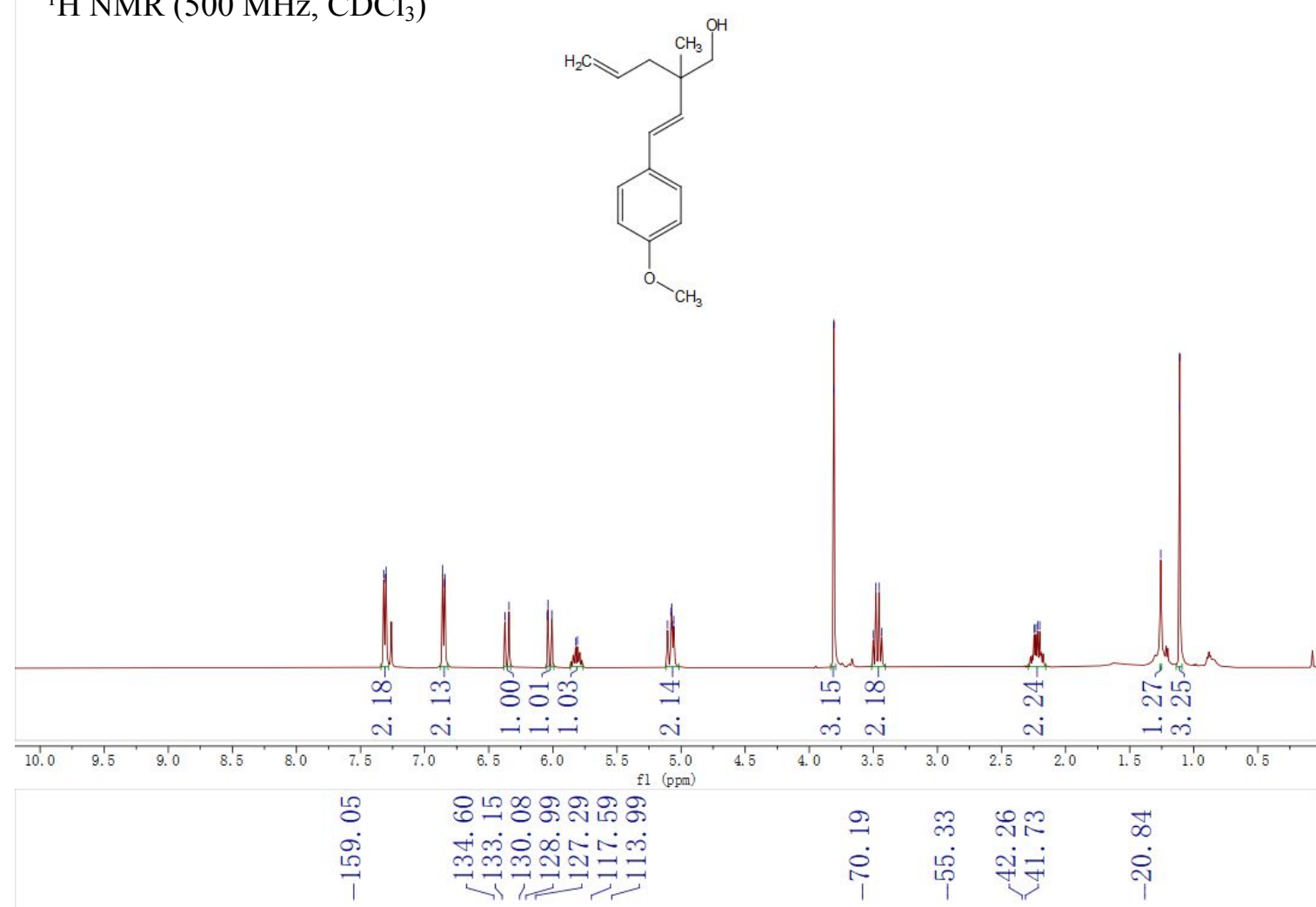

${ }^{13} \mathrm{C}\left\{{ }^{1} \mathrm{H}\right\}$ NMR $\left(126 \mathrm{MHz}, \mathrm{CDCl}_{3}\right)$
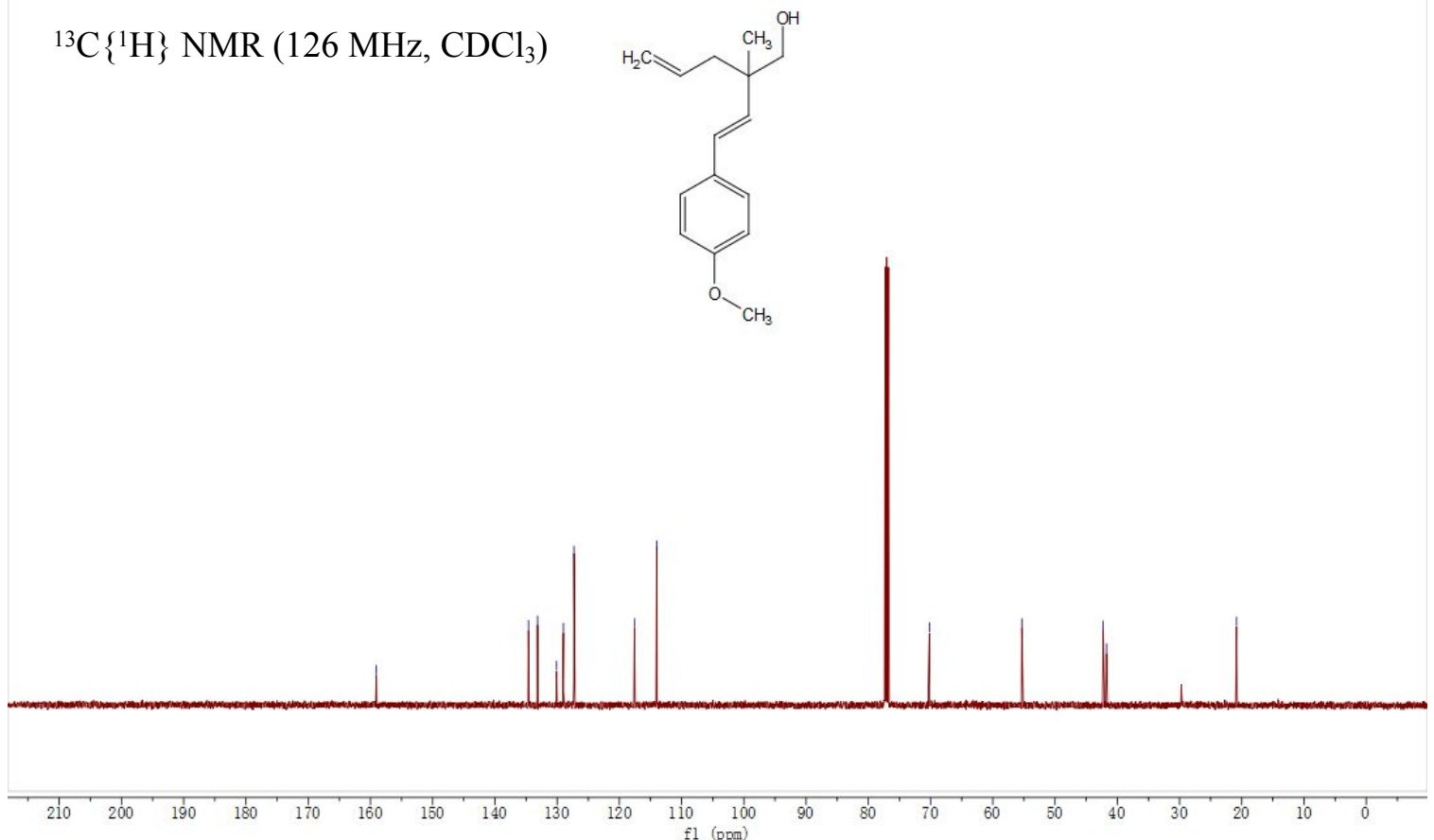

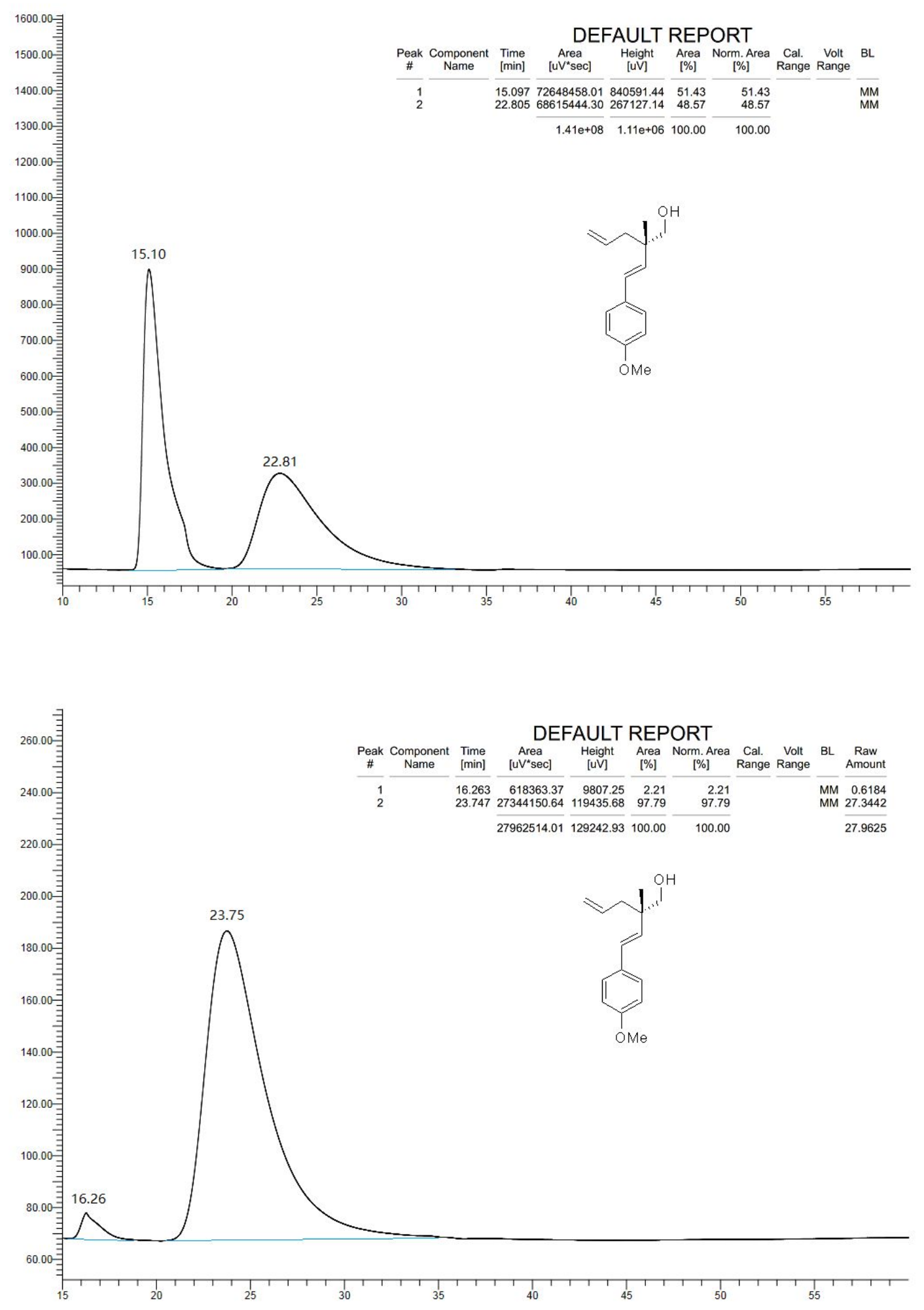


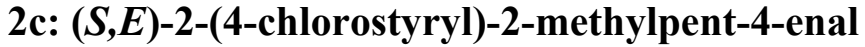

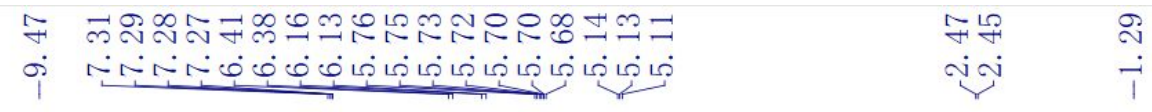

${ }^{1} \mathrm{H}$ NMR $\left(500 \mathrm{MHz}, \mathrm{CDCl}_{3}\right)$<smiles>C=CCC(C)(C=O)C=O</smiles>

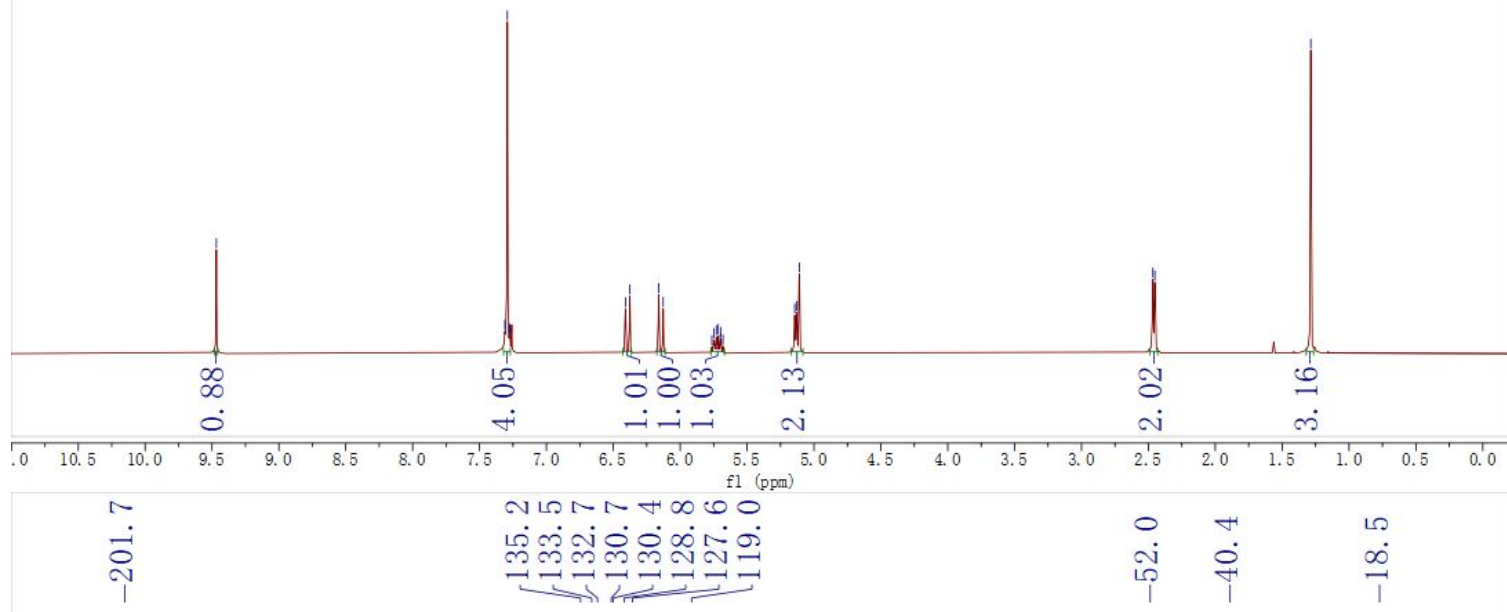

${ }^{13} \mathrm{C}\left\{{ }^{1} \mathrm{H}\right\} \mathrm{NMR}\left(126 \mathrm{MHz}, \mathrm{CDCl}_{3}\right)$
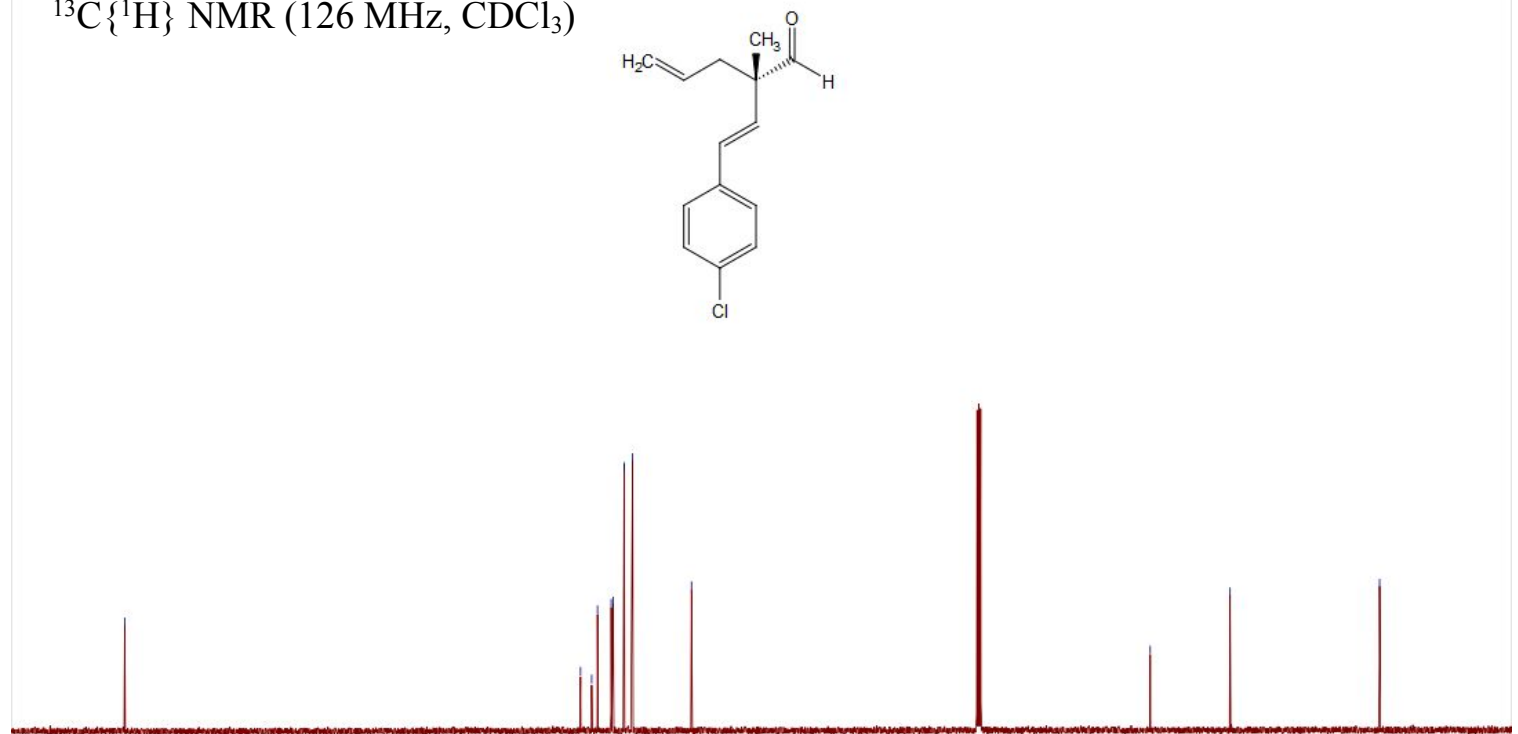

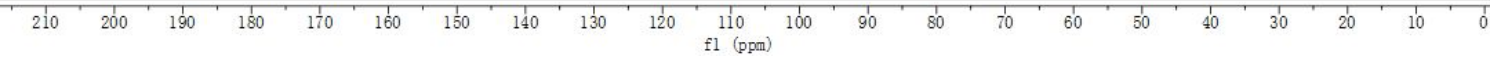



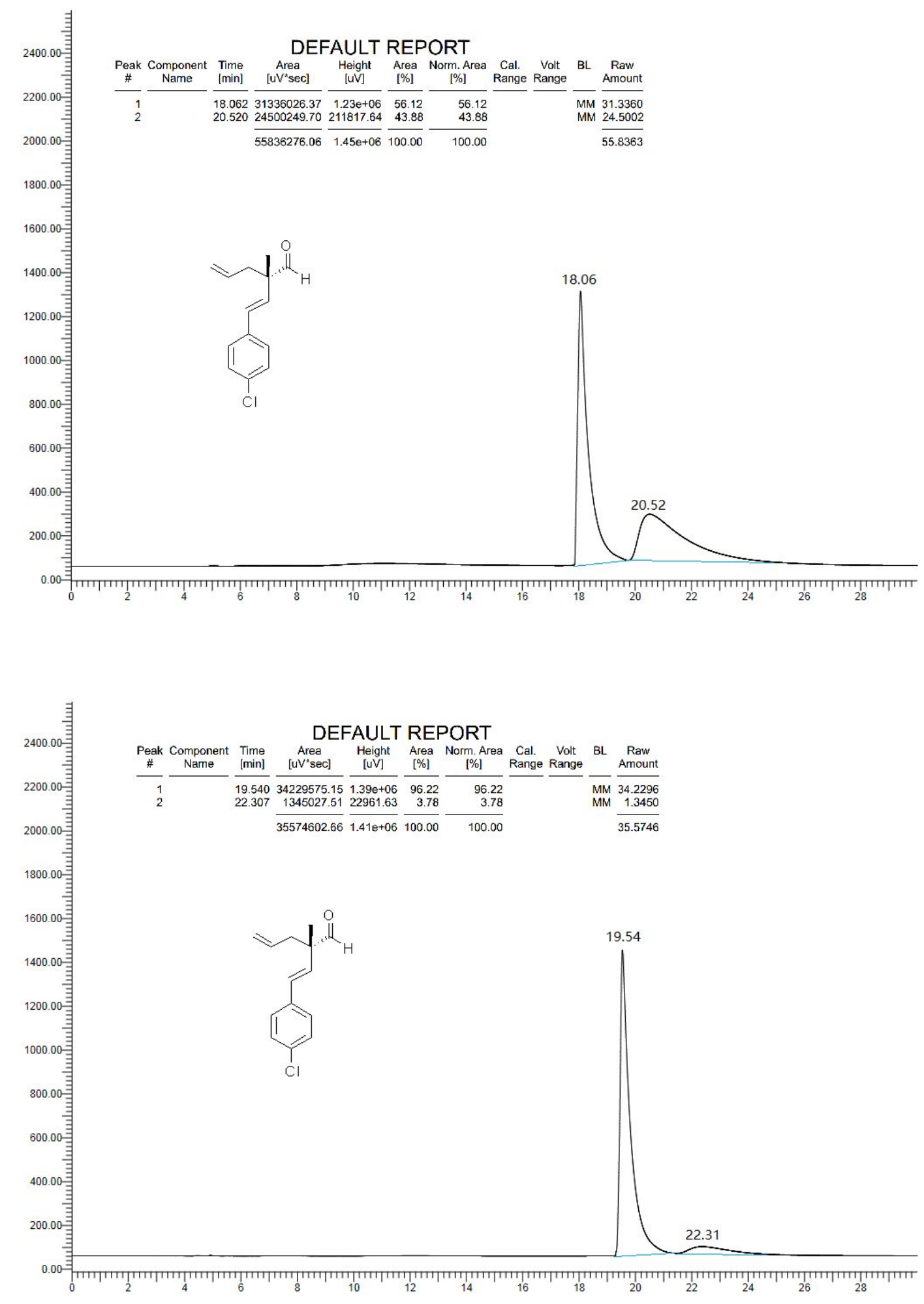
2d: (S,E)-2-(4-fluorostyryl)-2-methylpent-4-enal

F 以็

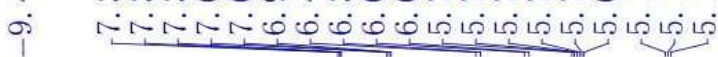

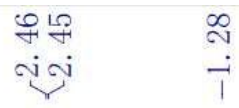

${ }^{1} \mathrm{H}$ NMR $\left(500 \mathrm{MHz}, \mathrm{CDCl}_{3}\right)$

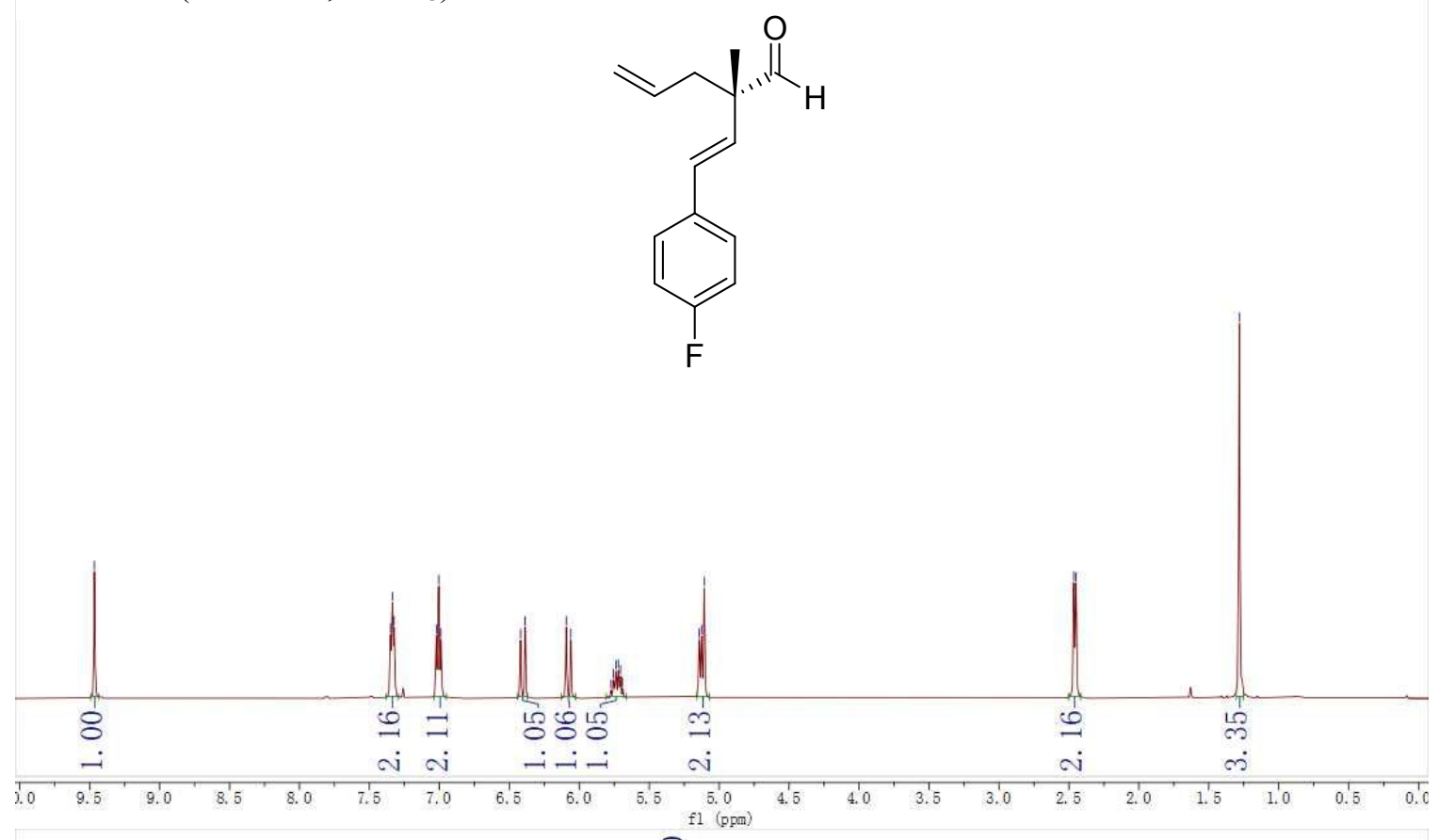

${ }^{19} \mathrm{~F}$ NMR (471 MHz, $\left.\mathrm{CDCl}_{3}\right)$
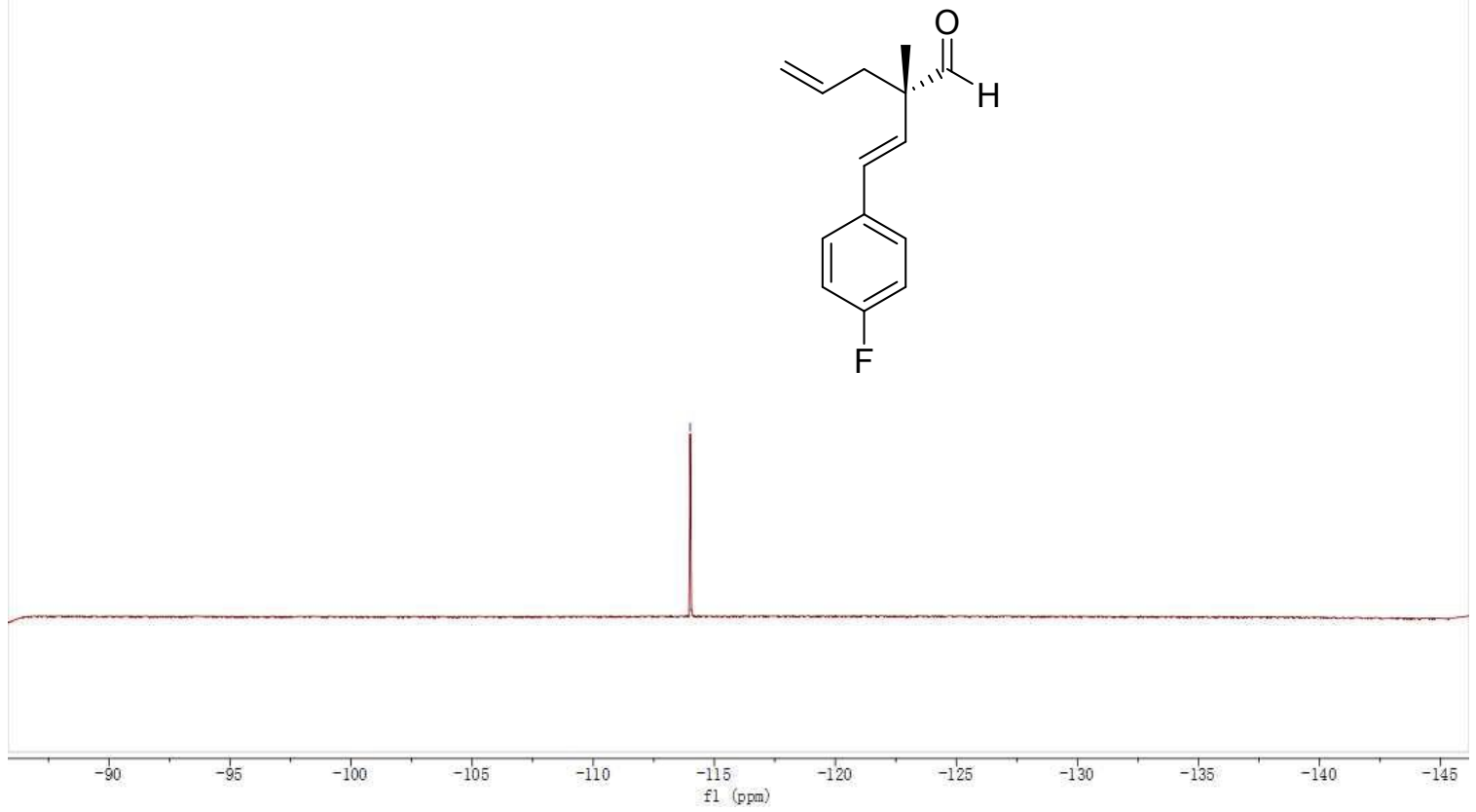

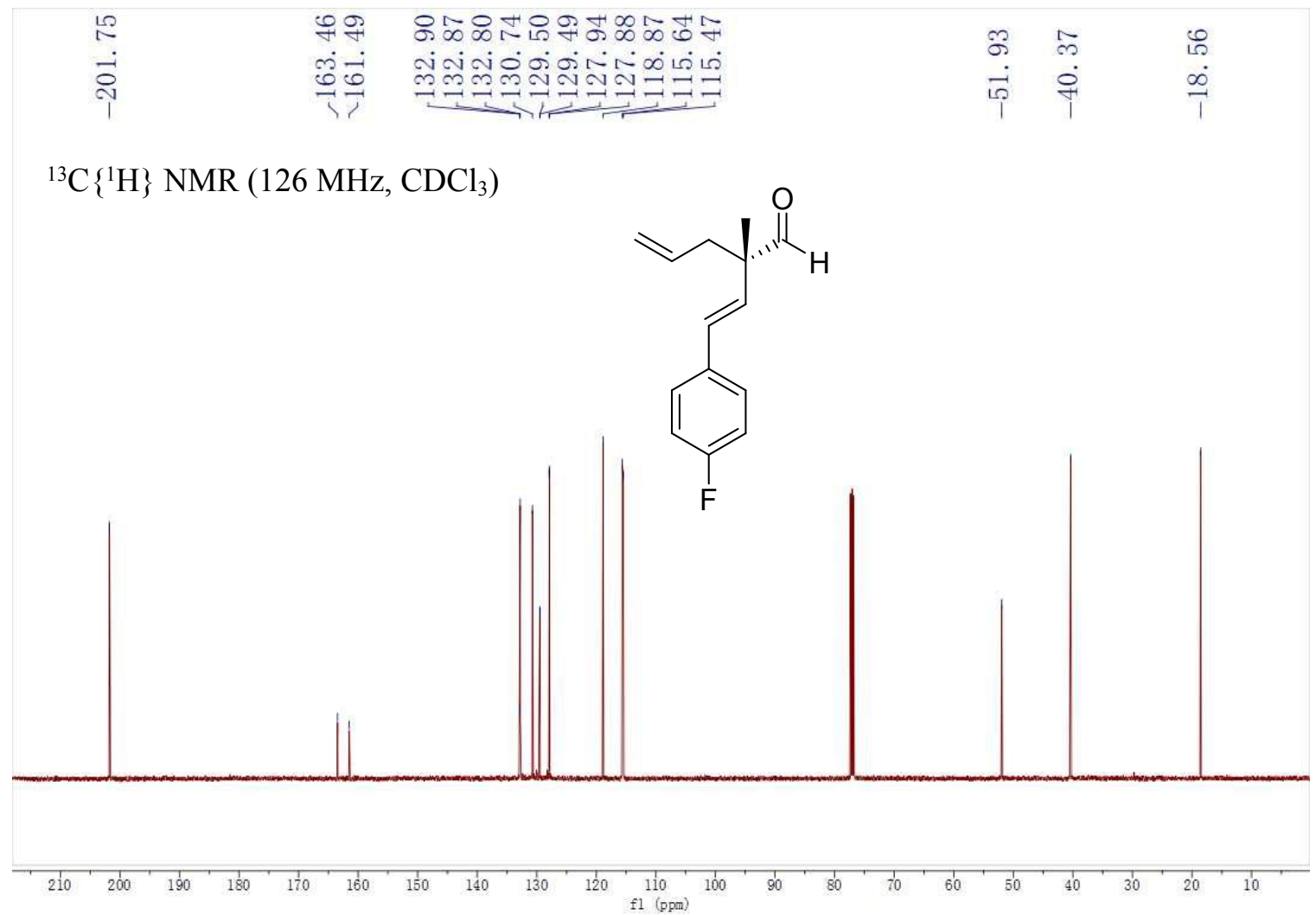
Corresponding alcohol of 2d: (S,E)-2-(4-fluorostyryl)-2-methylpent-4-en-1-ol

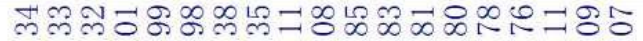

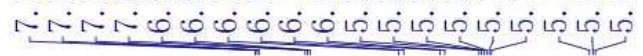

${ }^{1} \mathrm{H}$ NMR $\left(500 \mathrm{MHz}, \mathrm{CDCl}_{3}\right)$

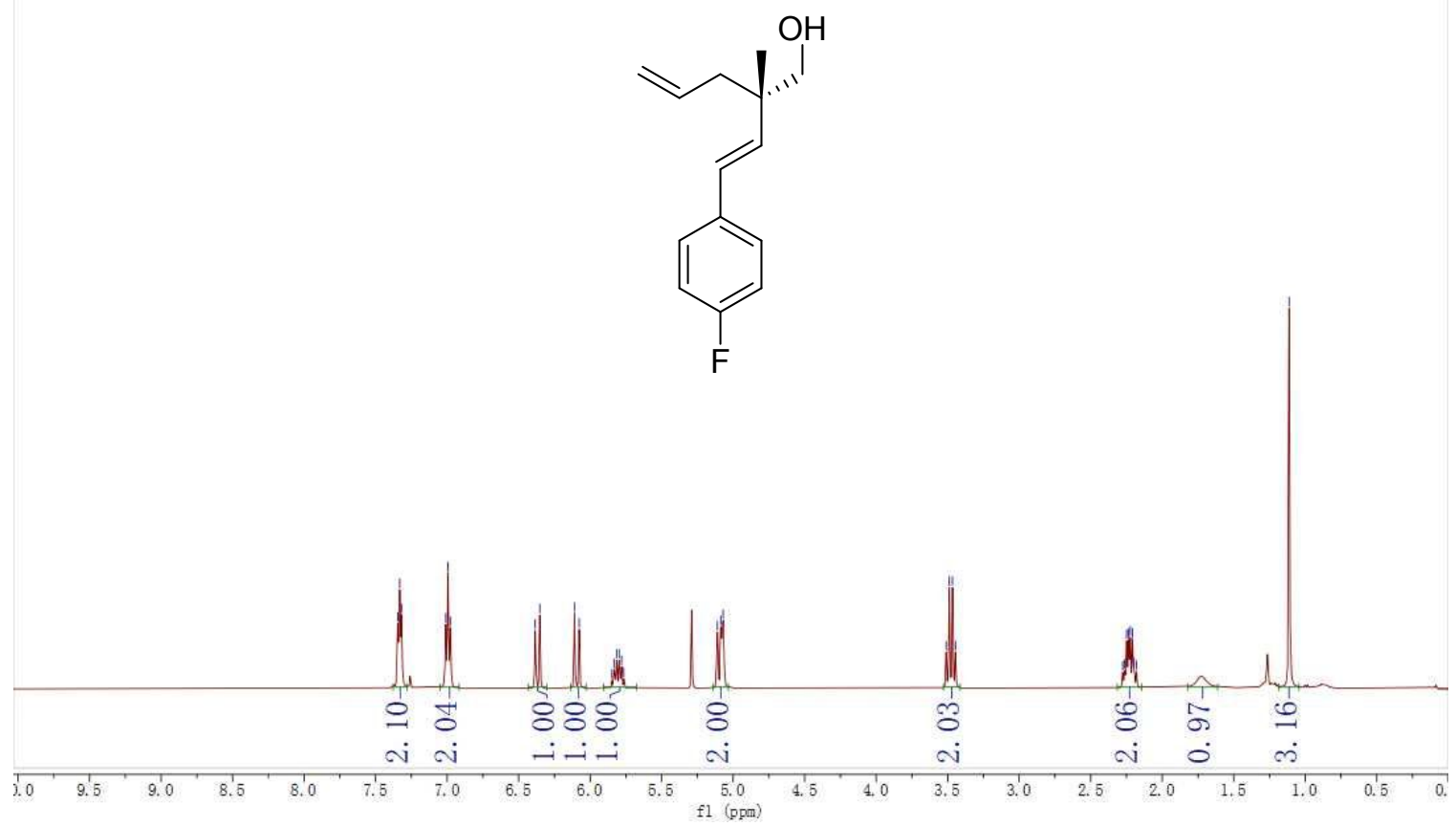




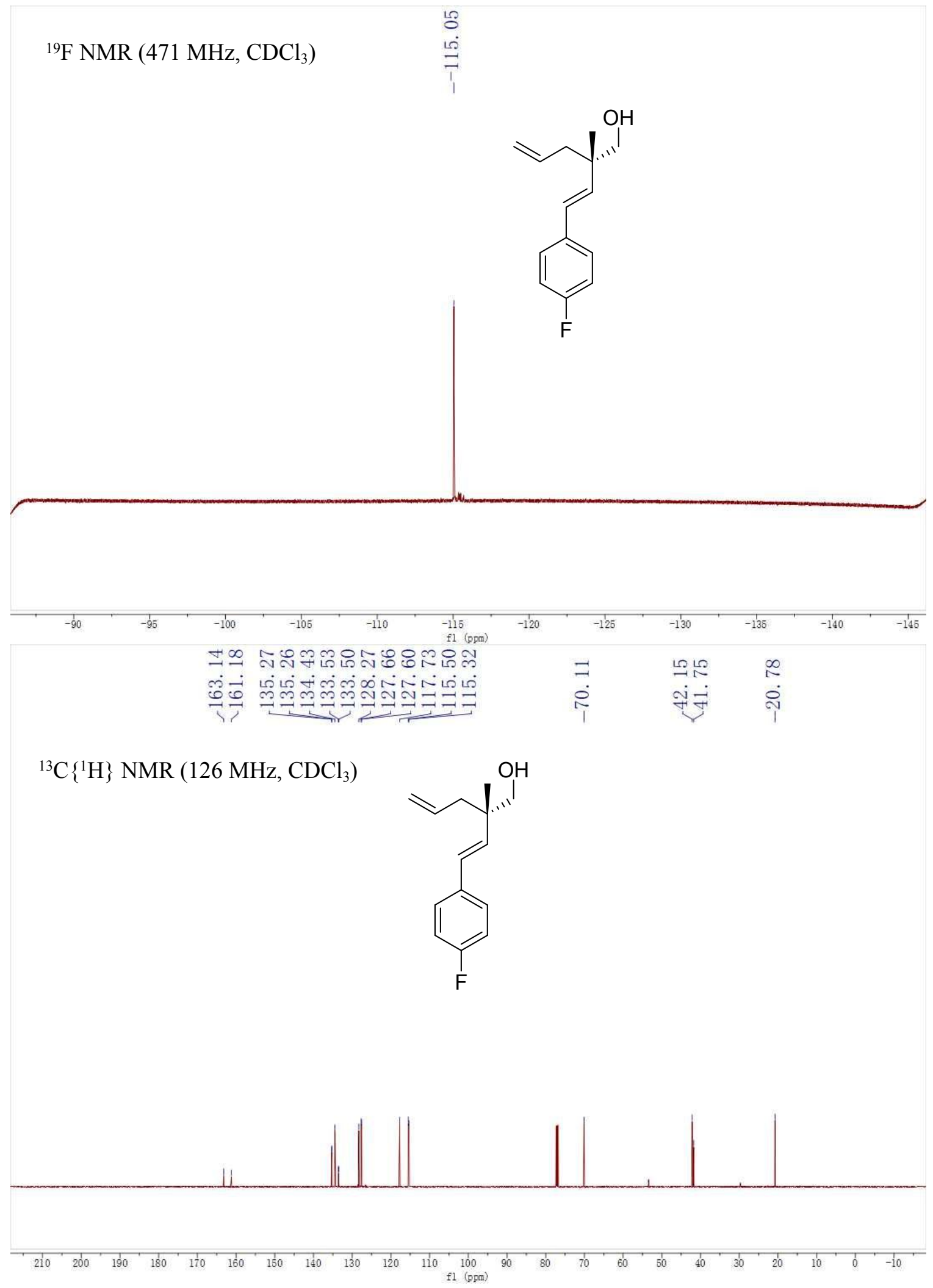



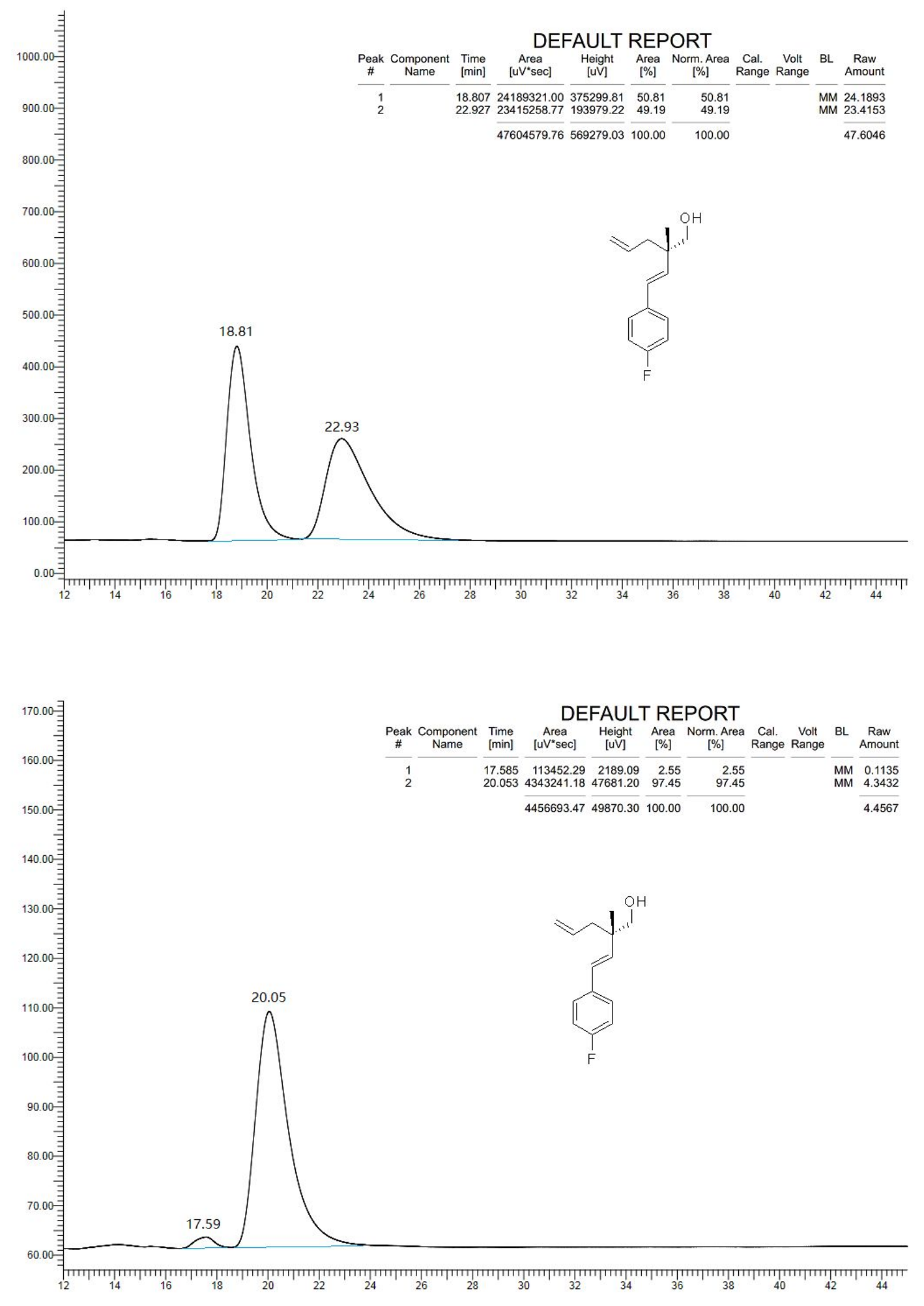


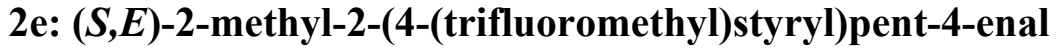

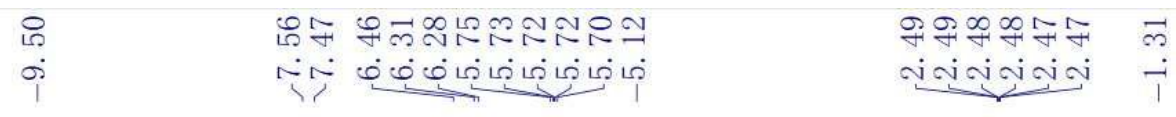

${ }^{1} \mathrm{H} \mathrm{NMR}\left(500 \mathrm{MHz}, \mathrm{CDCl}_{3}\right)$

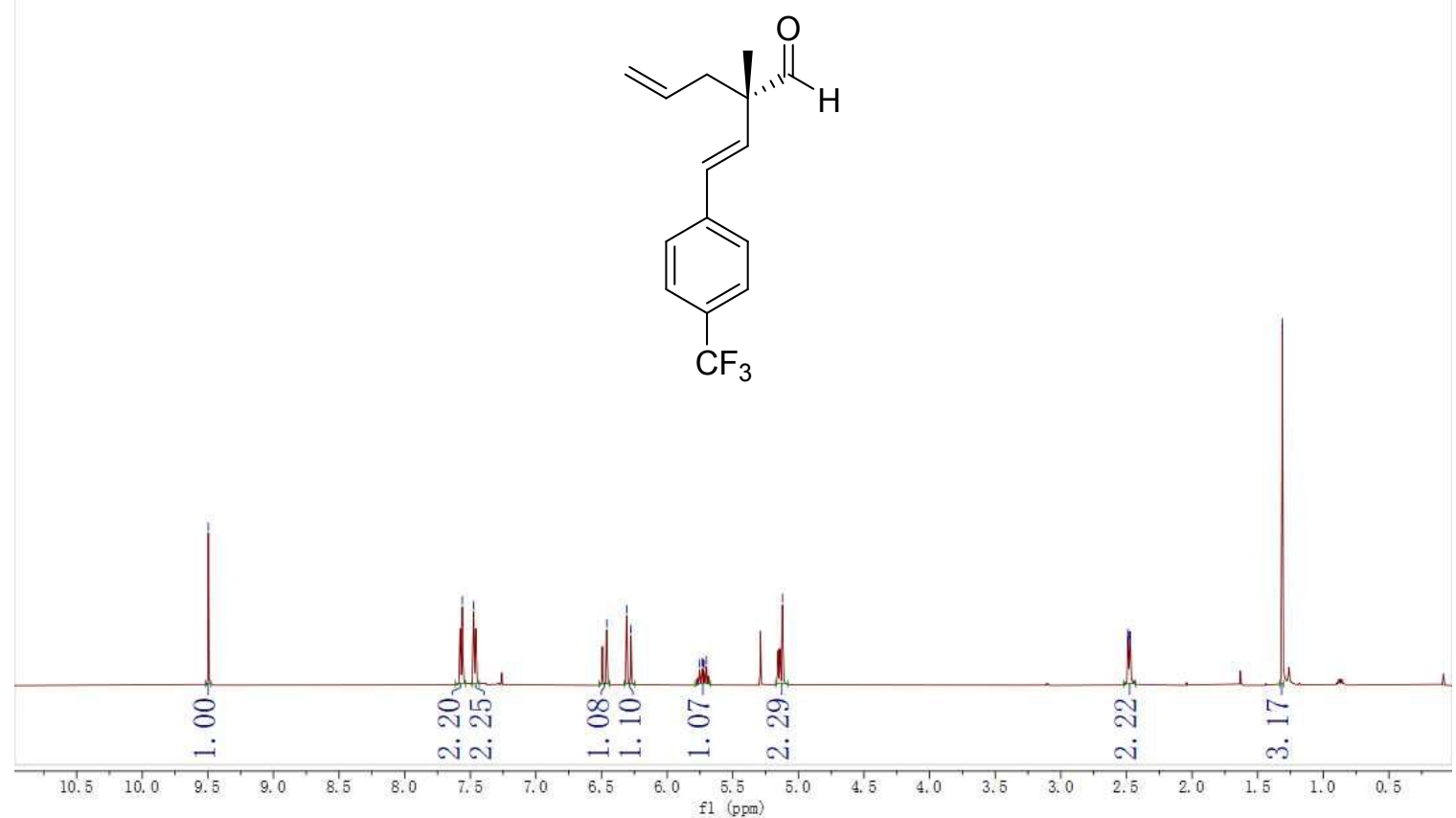


${ }^{19} \mathrm{~F}$ NMR (471 MHz, $\mathrm{CDCl}_{3}$ )
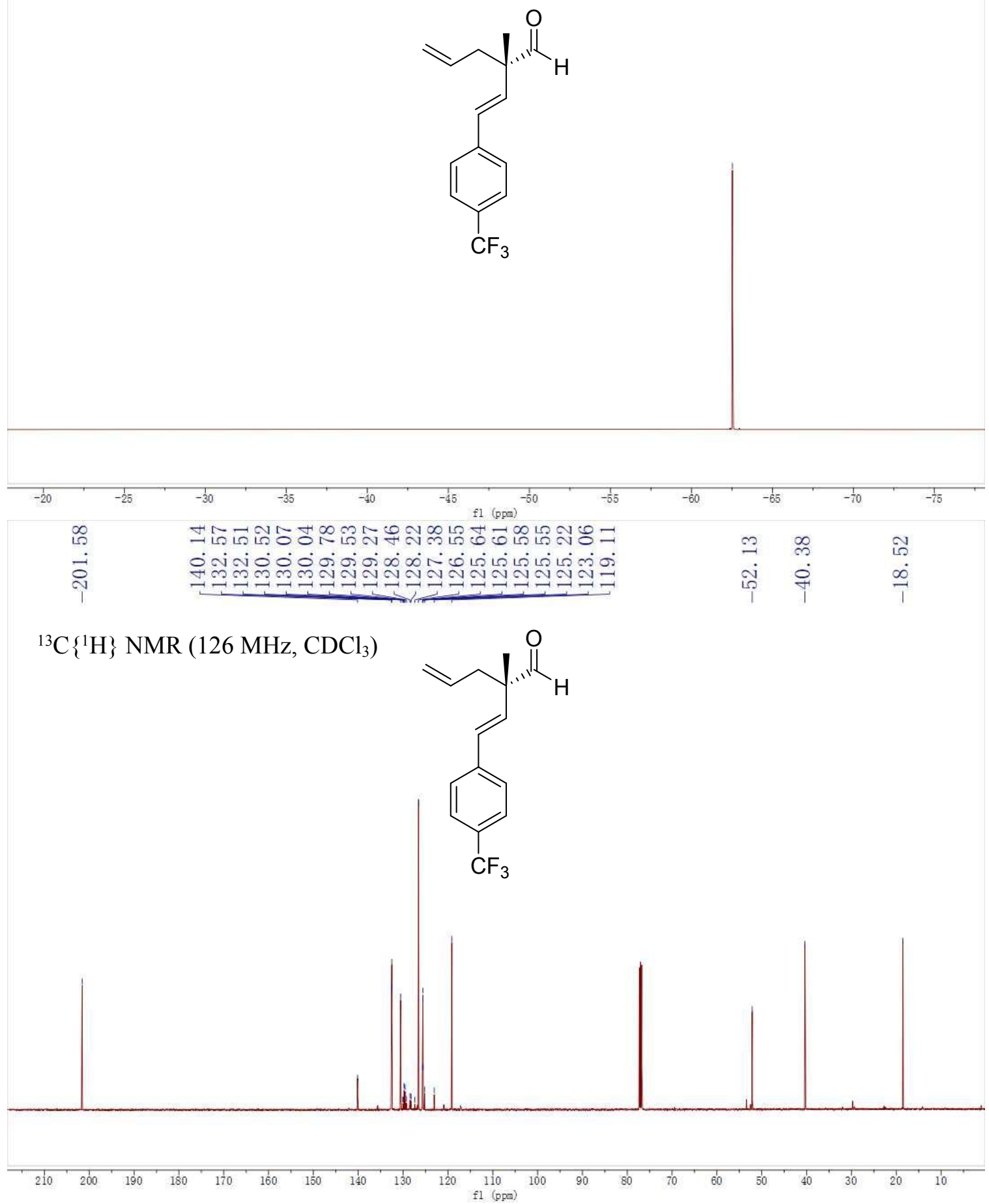
Corresponding alcohol of 2e: $(S, E)-2$-methyl-2-(4-(trifluoromethyl)styryl)pent-4-en1-ol

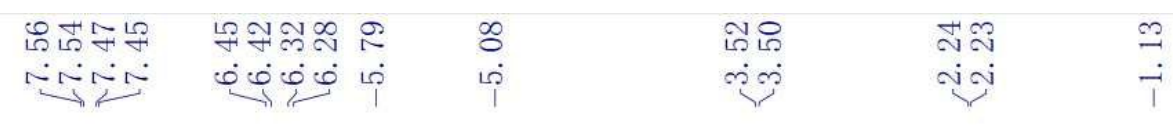

${ }^{1} \mathrm{H}$ NMR (500 MHz, $\mathrm{CDCl}_{3}$ )<smiles>C=CCC(C)(/C=C/c1ccc(C(F)(F)F)cc1)CO</smiles>

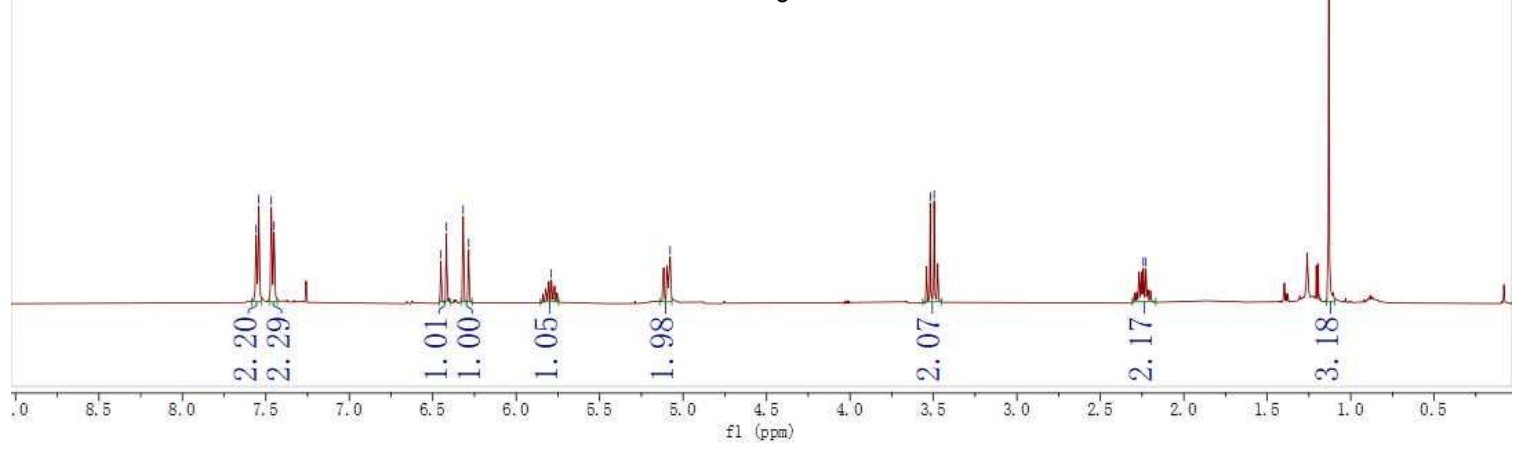


${ }^{19} \mathrm{~F}$ NMR (471 MHz, $\mathrm{CDCl}_{3}$ )
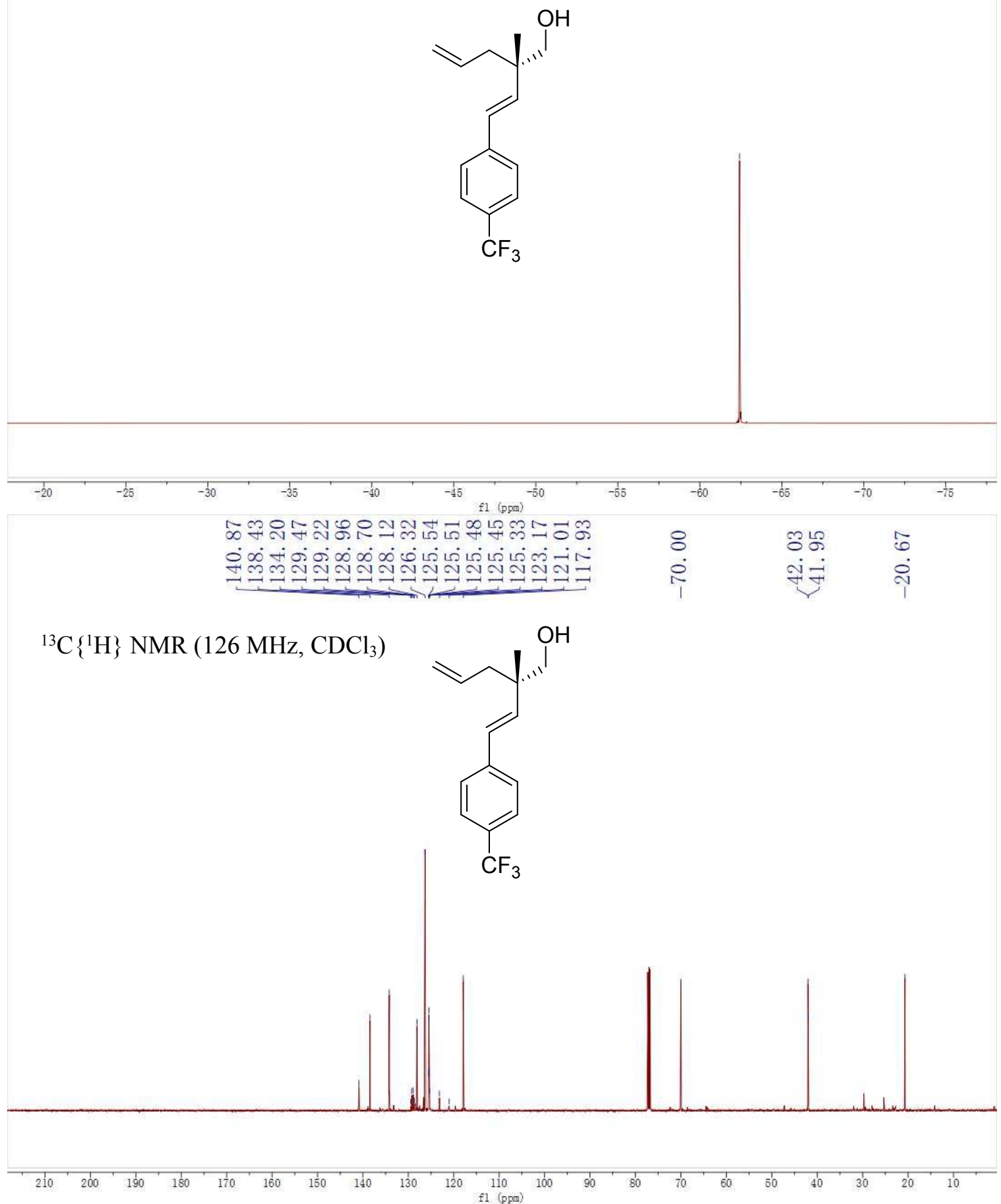

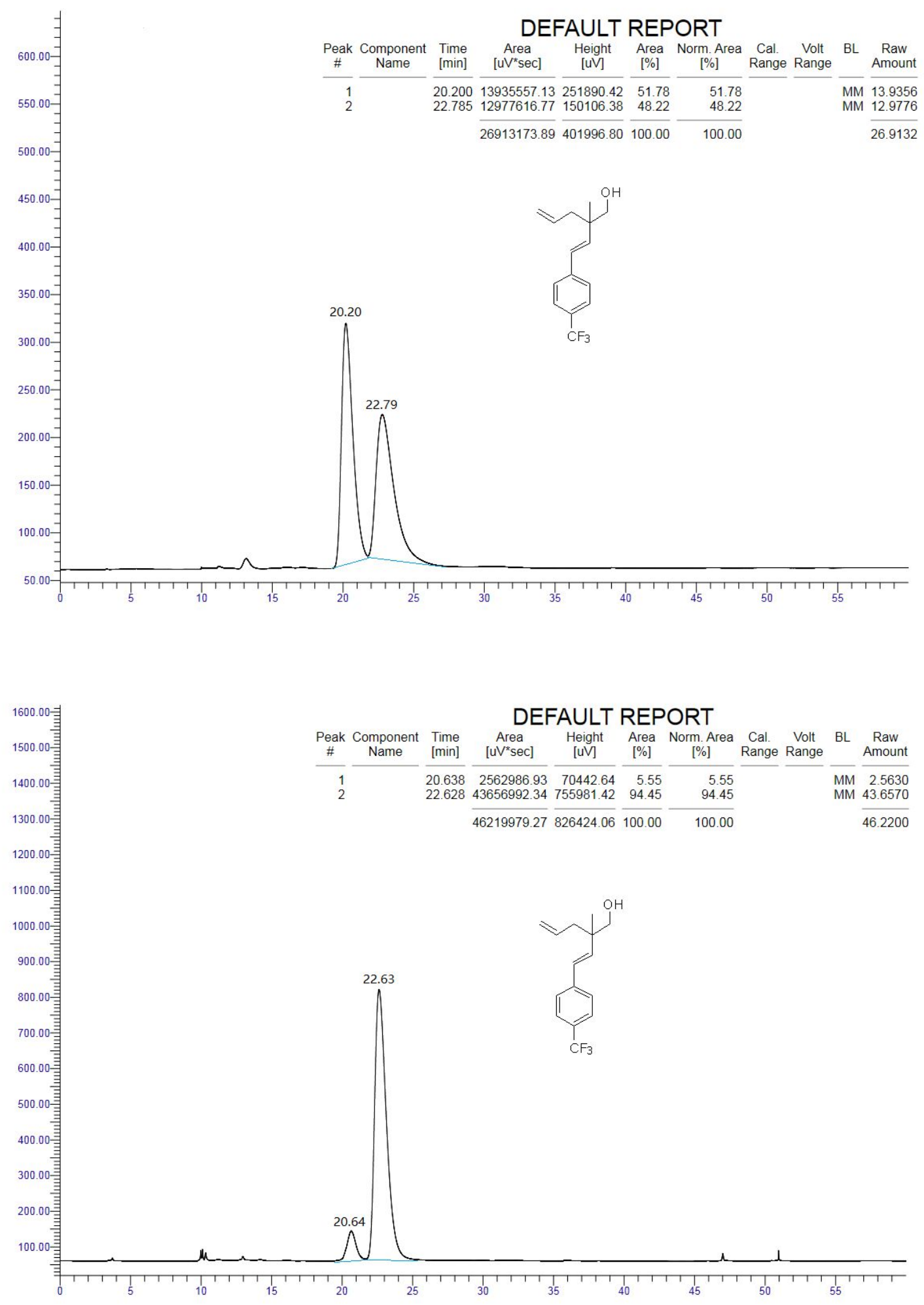
2e-- $\gamma$ : (E)-2-methyl-4-(4-(trifluoromethyl)phenyl)hepta-2,6-dienal

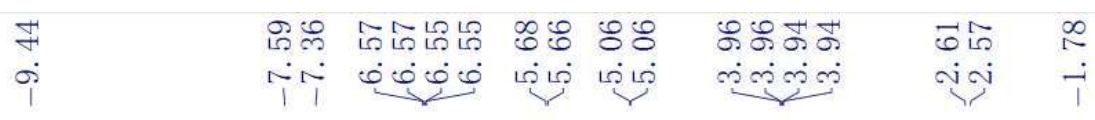

${ }^{1} \mathrm{H} \mathrm{NMR}\left(500 \mathrm{MHz}, \mathrm{CDCl}_{3}\right)$

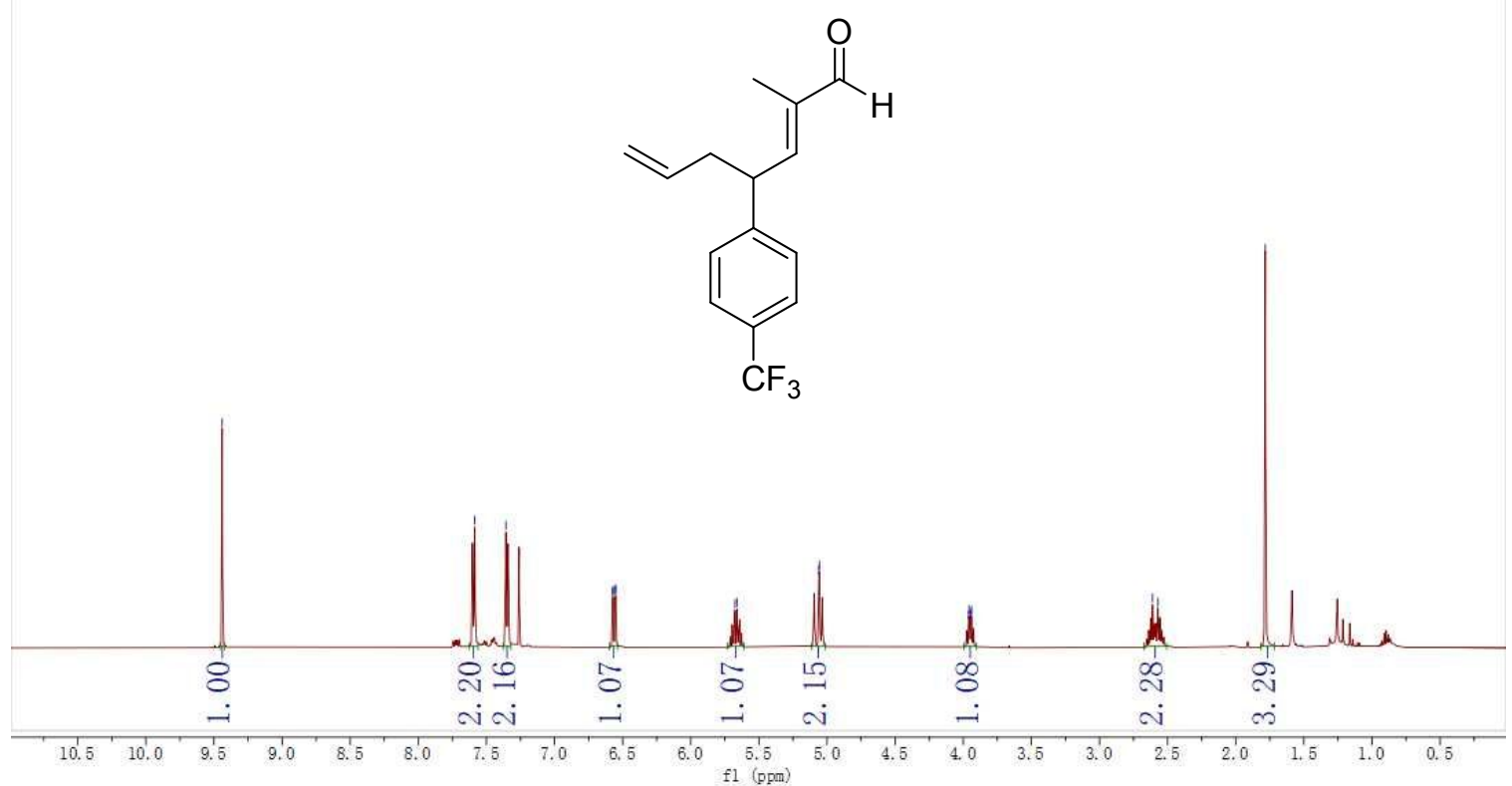


${ }^{19} \mathrm{~F}$ NMR (471 MHz, $\mathrm{CDCl}_{3}$ )<smiles>C=CCC(/C=C(\C)C=O)c1ccc(C(F)(F)F)cc1</smiles>
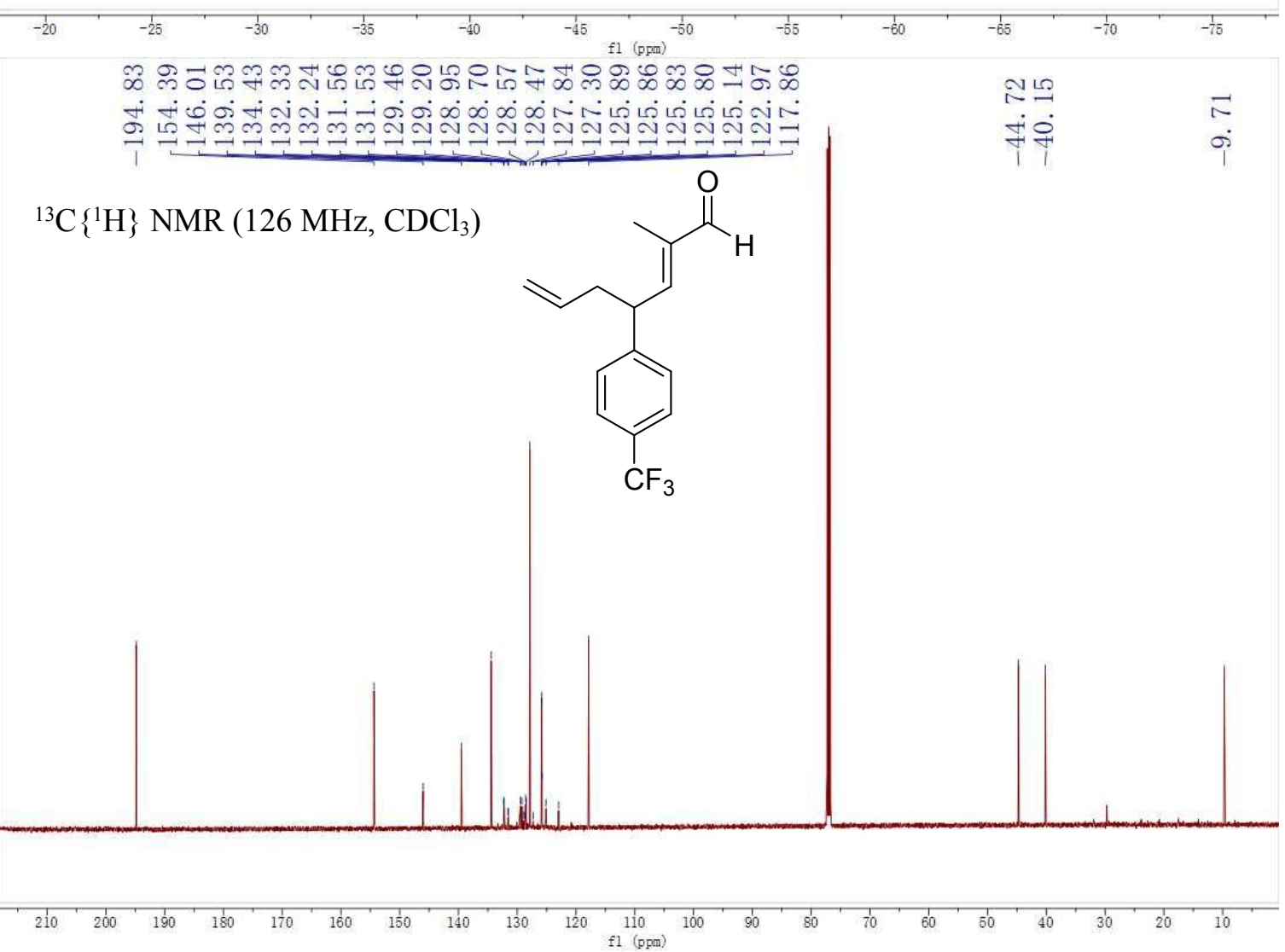


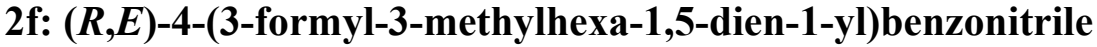

\begin{tabular}{|c|c|c|}
\hline g & 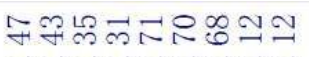 & 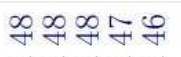 \\
\hline$\dot{\rho}$ & 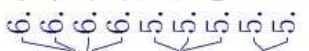 & ن่vivioi \\
\hline
\end{tabular}

${ }^{1} \mathrm{H}$ NMR $\left(500 \mathrm{MHz}, \mathrm{CDCl}_{3}\right)$<smiles>C=CCC(C)(C=O)/C=C/c1ccc(C#N)cc1</smiles>

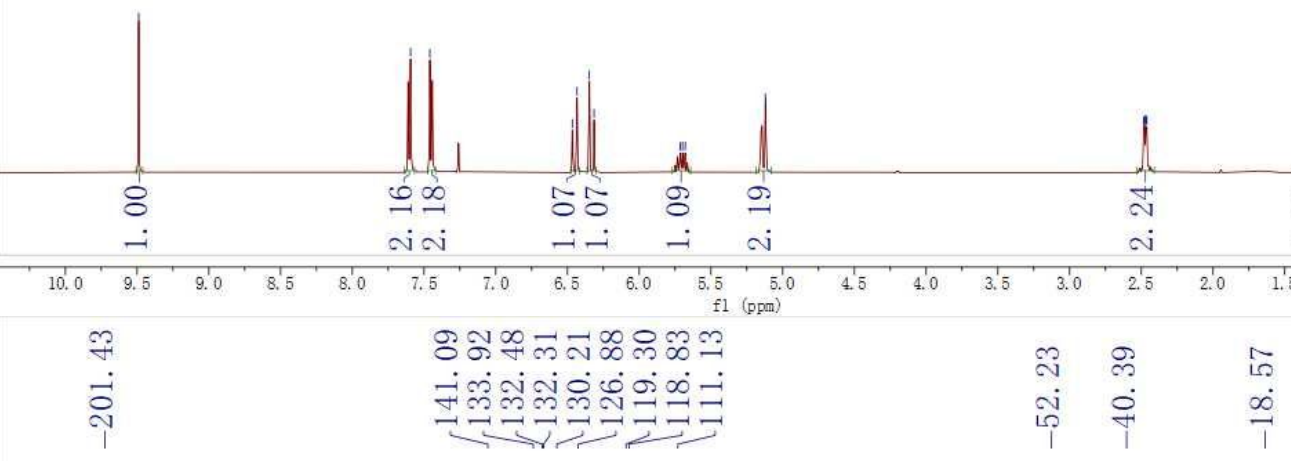

${ }^{13} \mathrm{C}\left\{{ }^{1} \mathrm{H}\right\}$ NMR $\left(126 \mathrm{MHz}, \mathrm{CDCl}_{3}\right)$

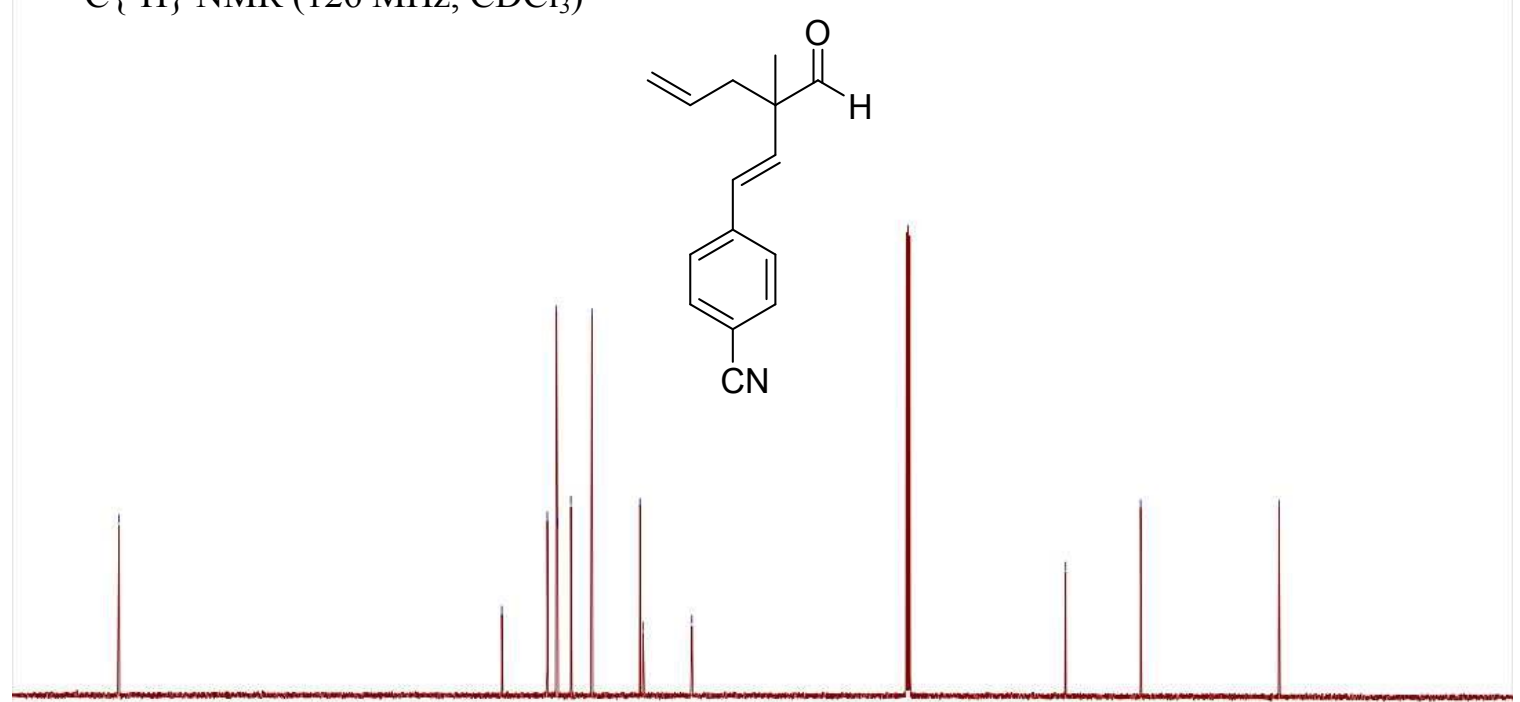

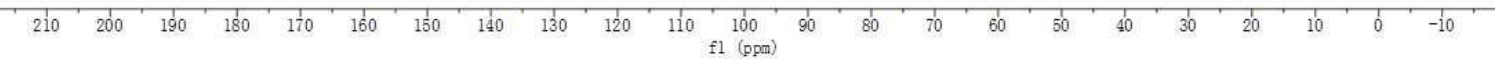




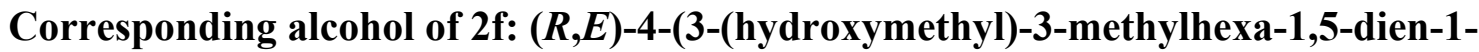
yl)benzonitrile

\begin{tabular}{|c|c|c|c|c|c|}
\hline 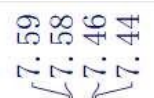 & $\begin{array}{l}\text { 유 } \\
\dot{0} 0\end{array}$ & $\begin{array}{l}2 \infty \\
200 \\
100\end{array}$ & $\begin{array}{l}8 \% \\
100 \\
100\end{array}$ & 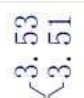 & 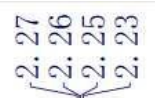 \\
\hline
\end{tabular}

${ }^{1} \mathrm{H}$ NMR $\left(500 \mathrm{MHz}, \mathrm{CDCl}_{3}\right)$
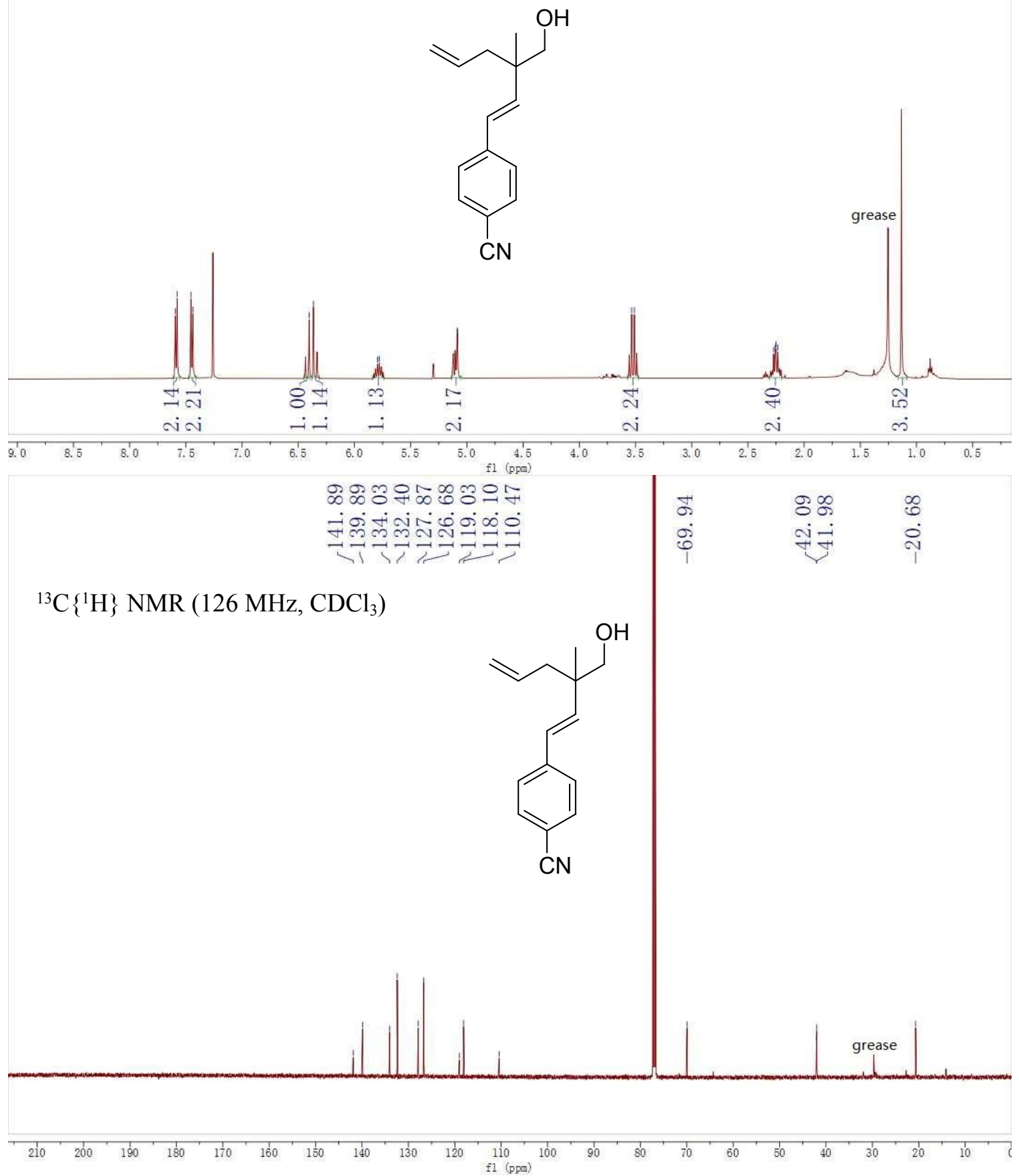

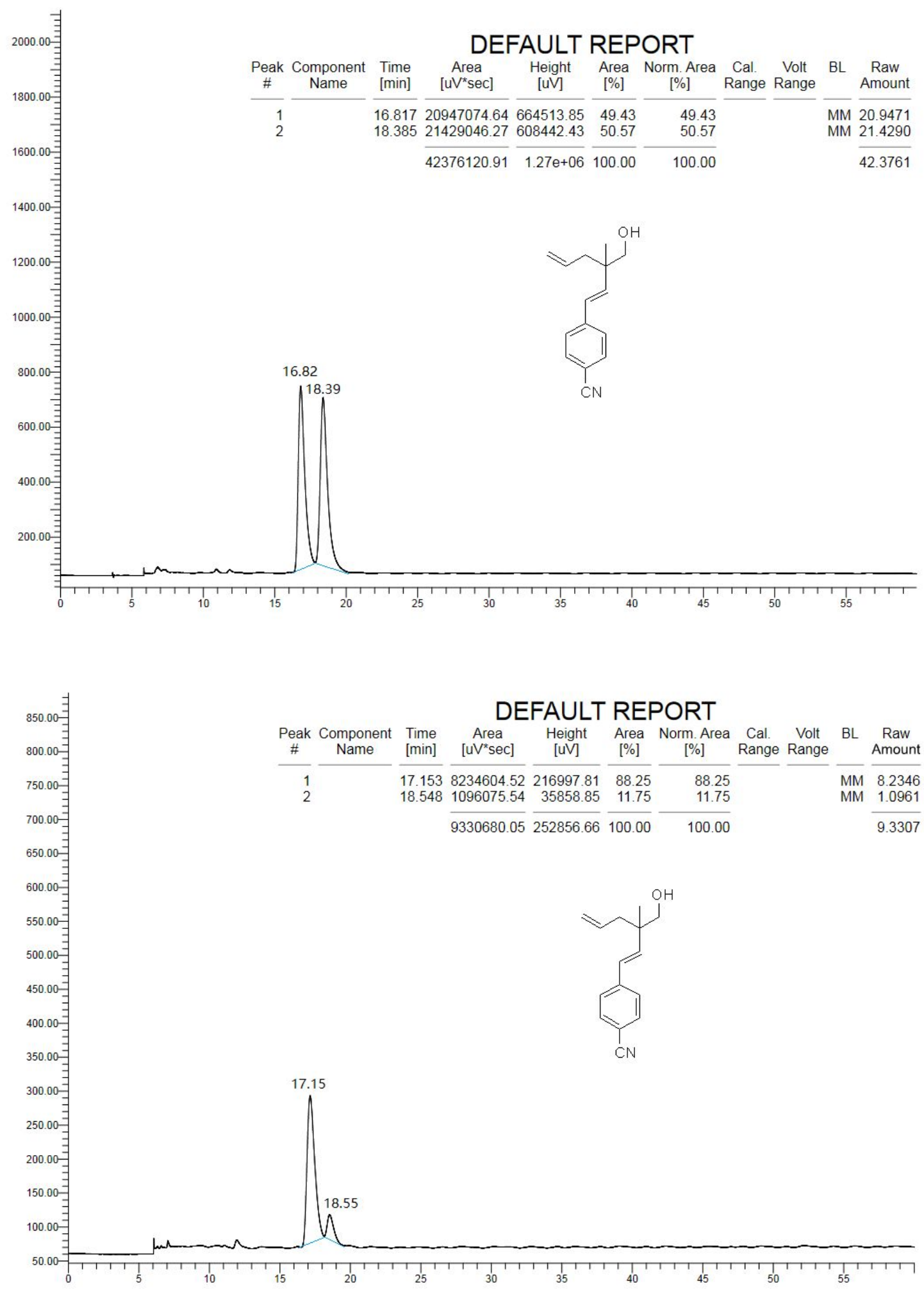
2f- $\gamma$ : (E)-4-(6-methyl-7-oxohepta-3-1,5-dien-4-yl)benzonitrile

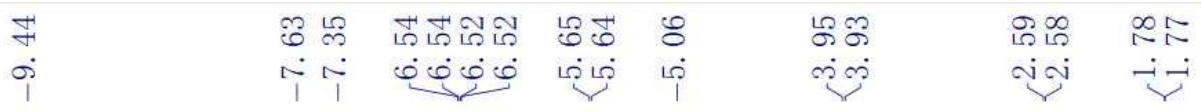

${ }^{1} \mathrm{H} \mathrm{NMR}\left(500 \mathrm{MHz}, \mathrm{CDCl}_{3}\right)$<smiles>C=CCC(/C=C/C(C)=O)c1ccc(C#N)cc1</smiles>

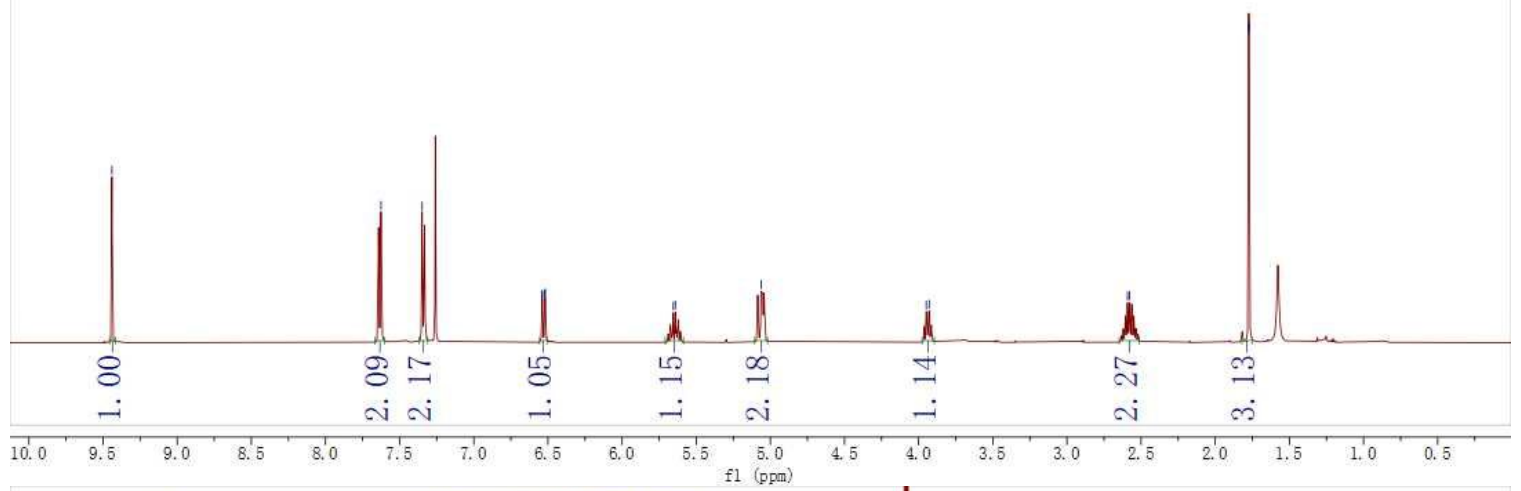

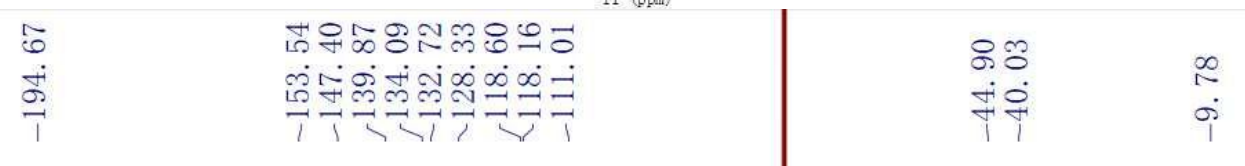

${ }^{13} \mathrm{C}\left\{{ }^{1} \mathrm{H}\right\}$ NMR $\left(126 \mathrm{MHz}, \mathrm{CDCl}_{3}\right)$<smiles>C=CCC(/C=C/C(C)=O)c1ccc(C#N)cc1</smiles>
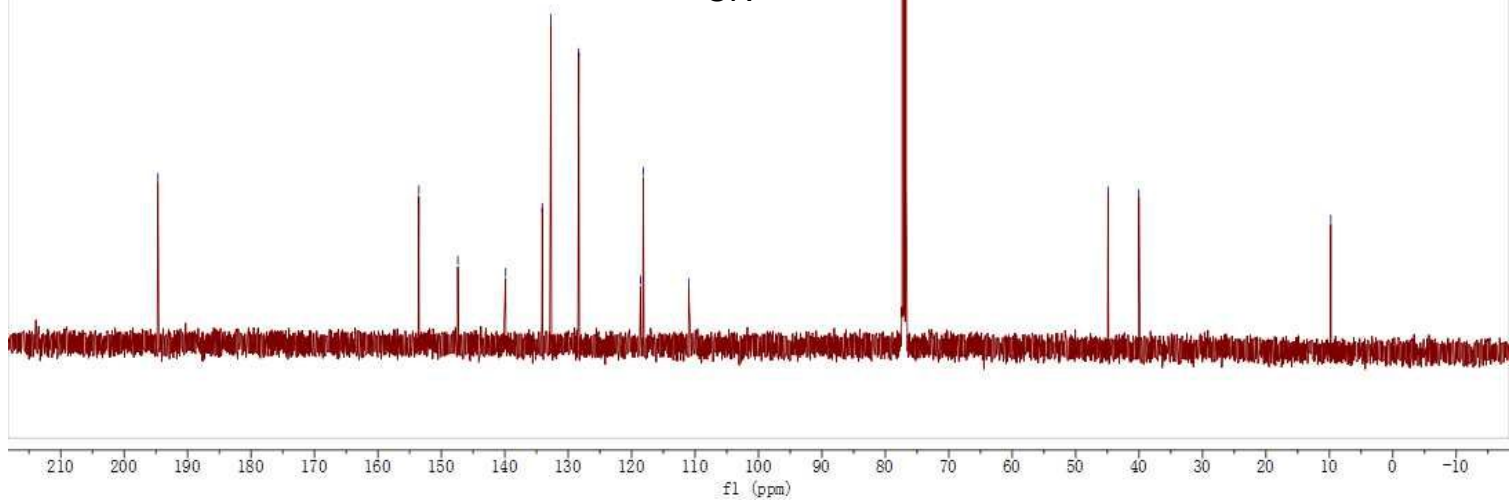
2g: (E)-2-methyl-2-(4-nitrostyryl)pent-4-enal

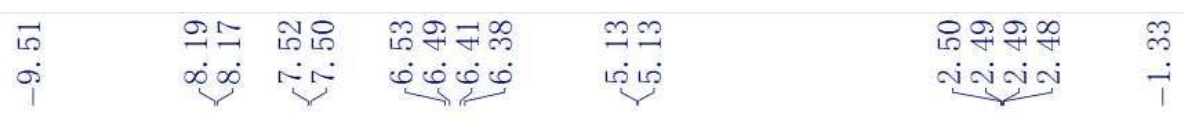

${ }^{1} \mathrm{H}$ NMR $\left(500 \mathrm{MHz}, \mathrm{CDCl}_{3}\right)$<smiles>C=CCC(C)(C=O)/C=C/c1ccc([N+](=O)[O-])cc1</smiles>

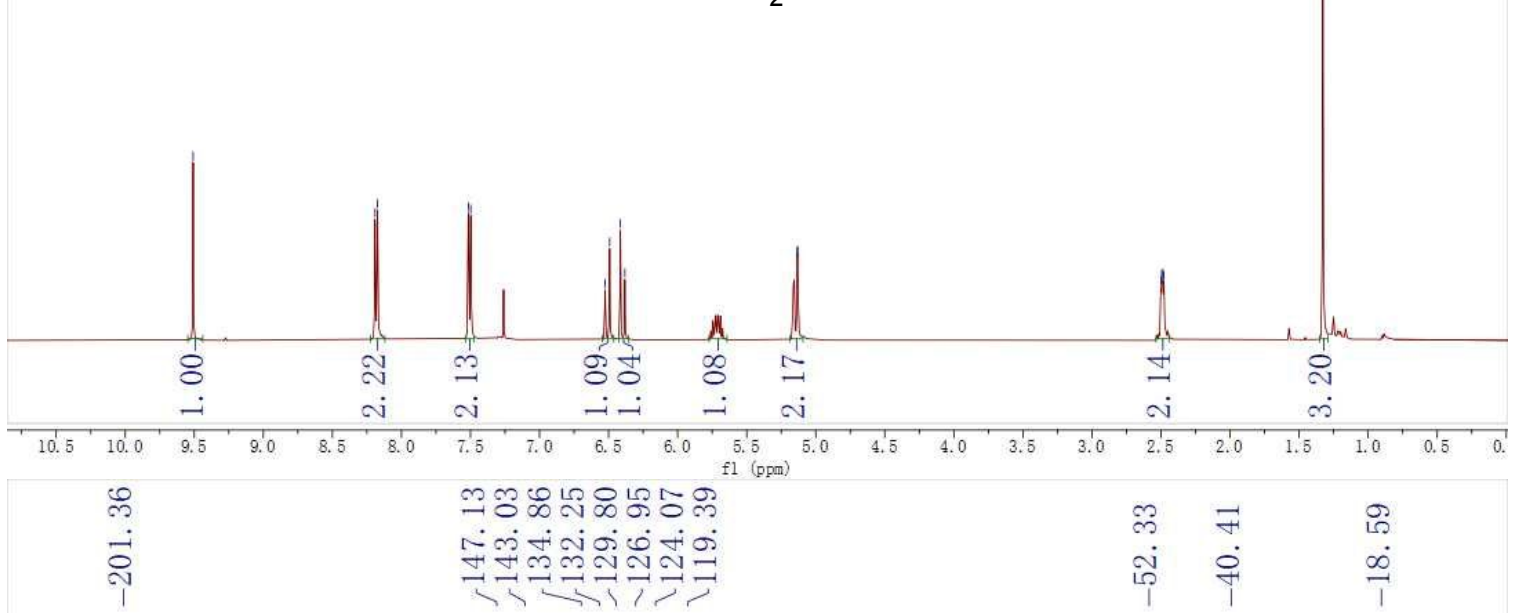

${ }^{13} \mathrm{C}\left\{{ }^{1} \mathrm{H}\right\} \mathrm{NMR}\left(126 \mathrm{MHz}, \mathrm{CDCl}_{3}\right)$<smiles>C=CCC(C)(C=O)/C=C/c1ccc([N+](=O)[O-])cc1</smiles>

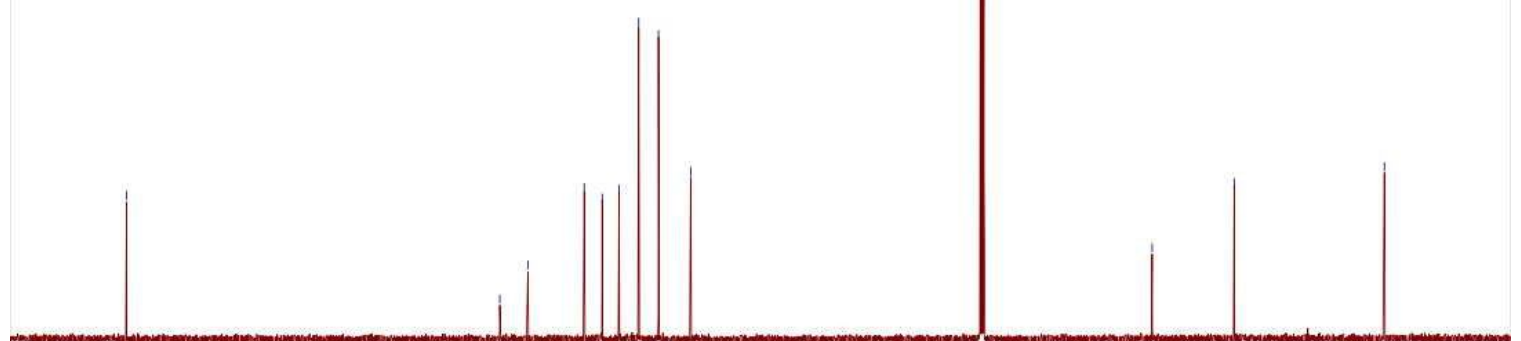

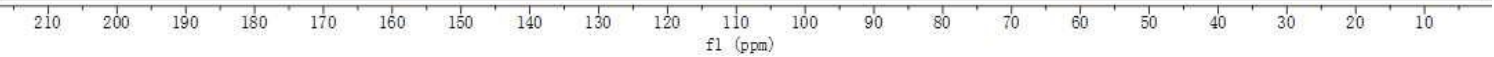



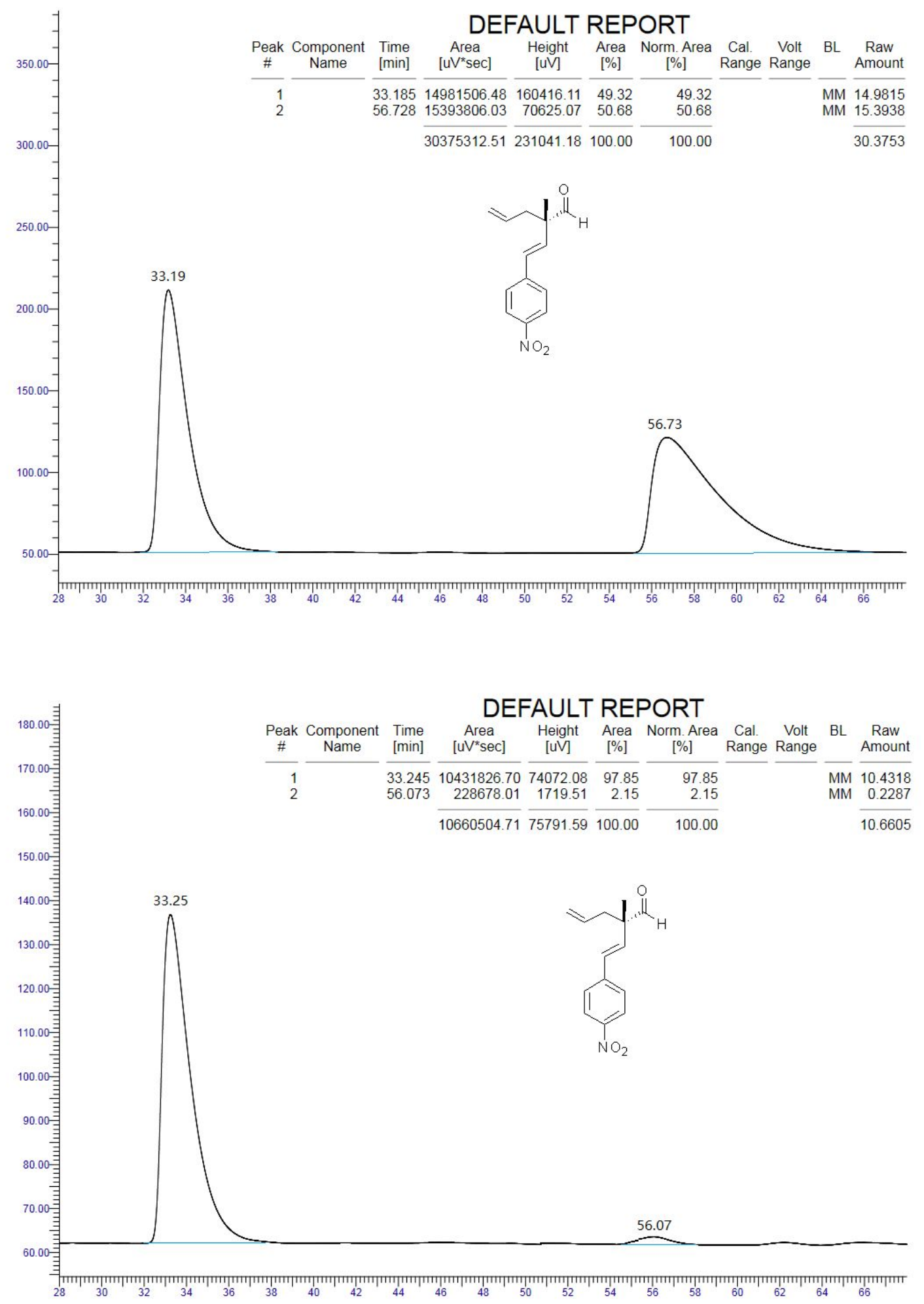


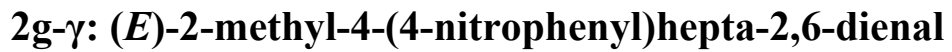

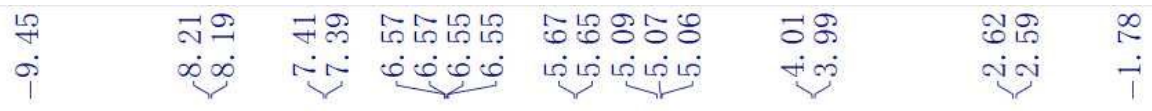

${ }^{1} \mathrm{H}$ NMR $\left(500 \mathrm{MHz}, \mathrm{CDCl}_{3}\right)$<smiles>C=CCC(/C=C(\C)C=O)c1ccc([N+](=O)[O-])cc1</smiles>

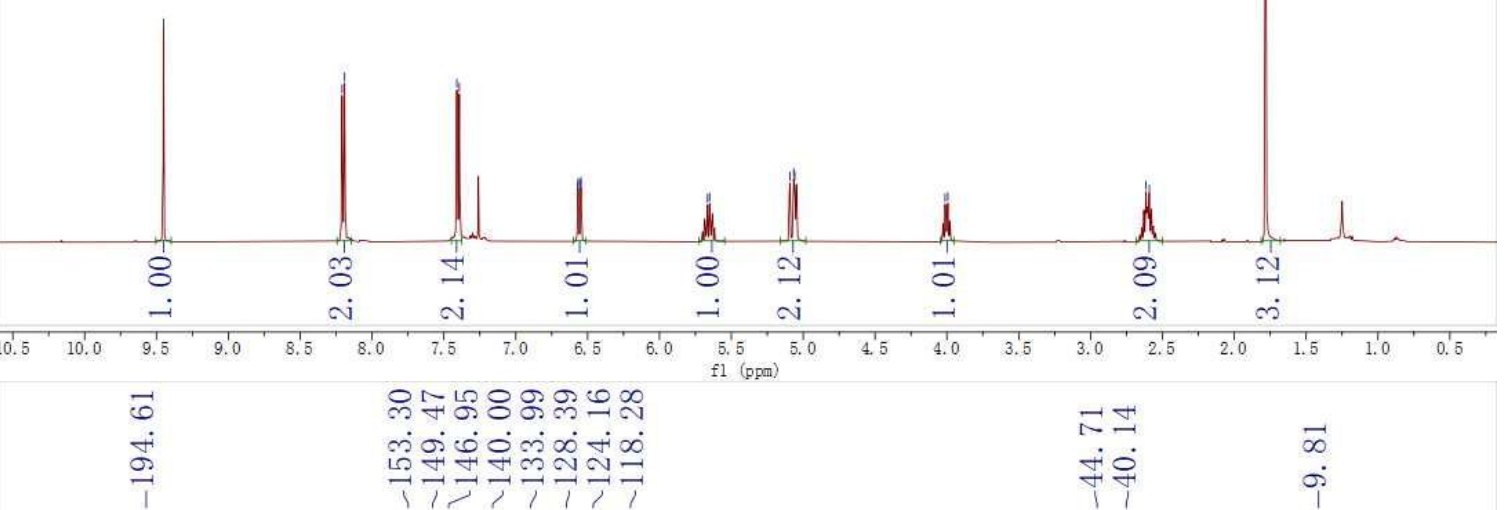

${ }^{13} \mathrm{C}\left\{{ }^{1} \mathrm{H}\right\}$ NMR $\left(126 \mathrm{MHz}, \mathrm{CDCl}_{3}\right)$<smiles>C=CCC(/C=C(\C)C=O)c1ccc([N+](=O)[O-])cc1</smiles>
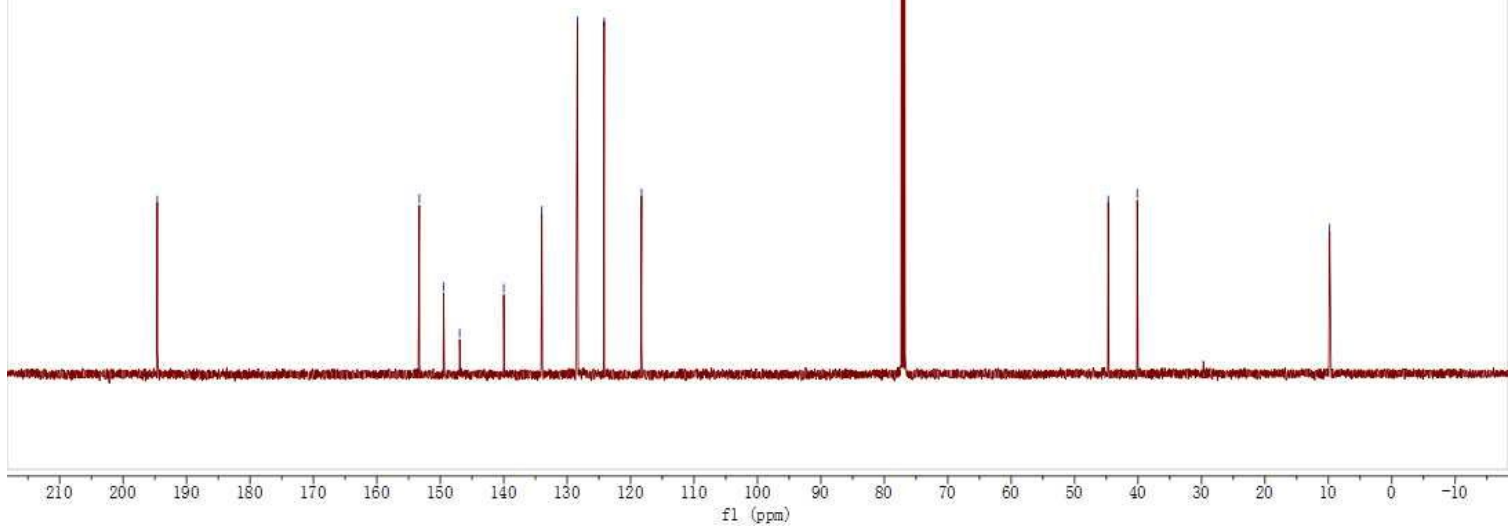
2h: (S,E)-2-methyl-2-(2-(trifluoromethyl)styryl)pent-4-enal

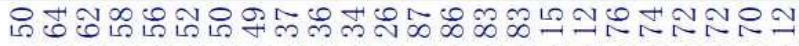

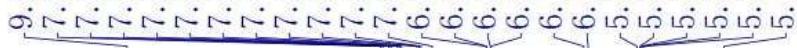

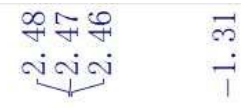

${ }^{1} \mathrm{H}$ NMR $\left(500 \mathrm{MHz}, \mathrm{CDCl}_{3}\right)$

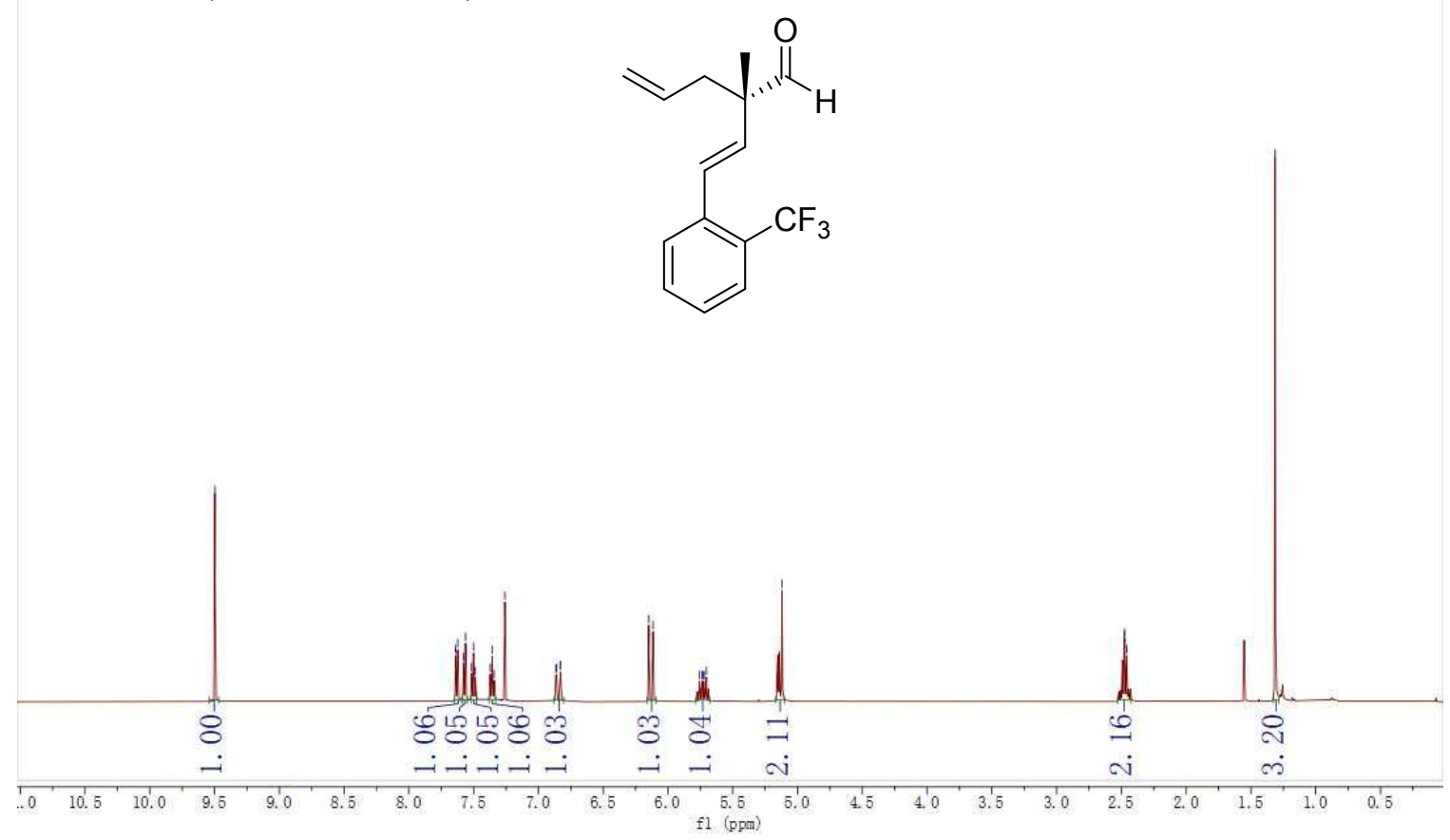


${ }^{19} \mathrm{~F}$ NMR $\left(471 \mathrm{MHz}, \mathrm{CDCl}_{3}\right)$<smiles>C=CC[C@@](C)(C=O)/C=C/c1ccccc1C(F)(F)F</smiles>
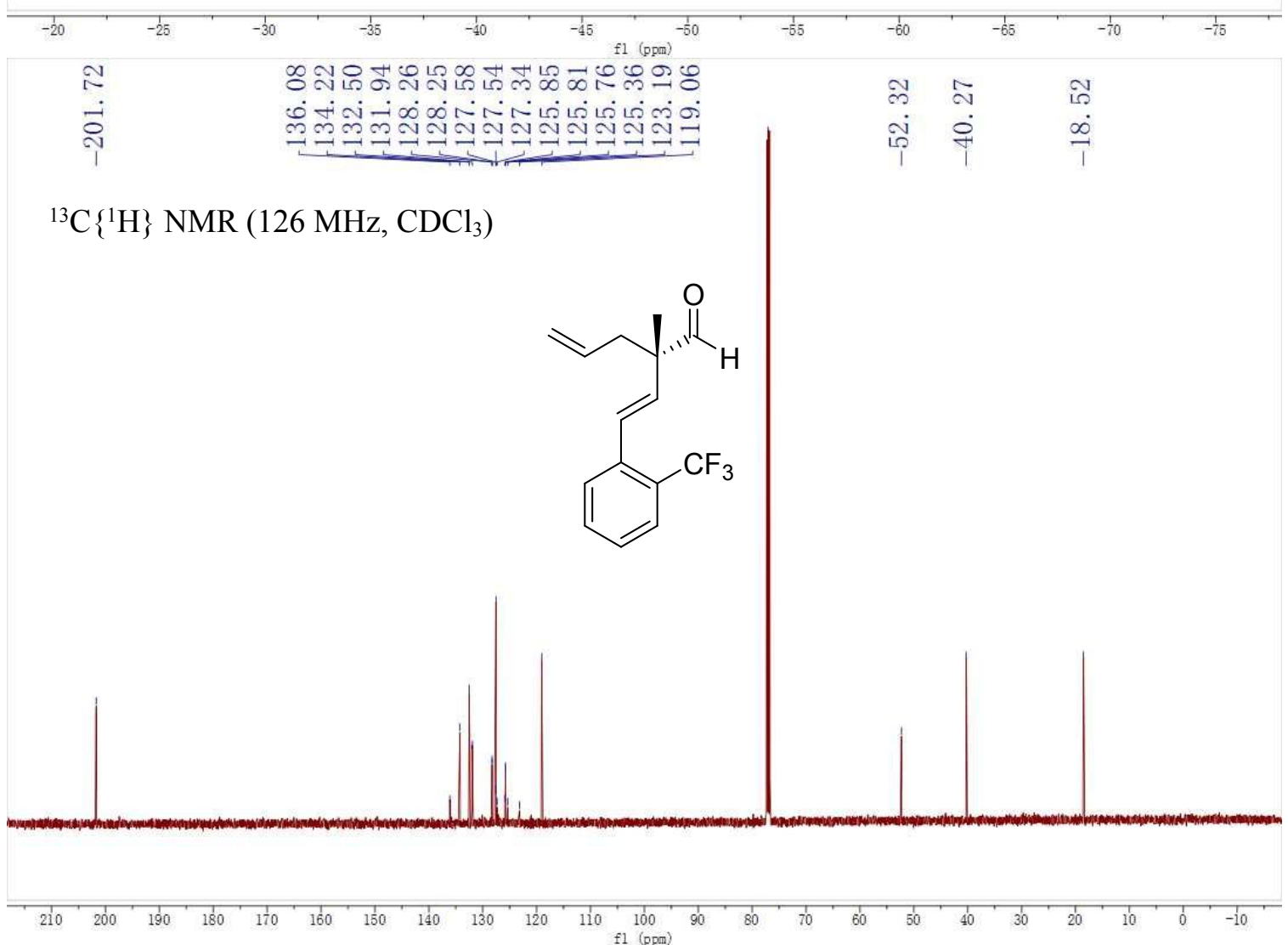

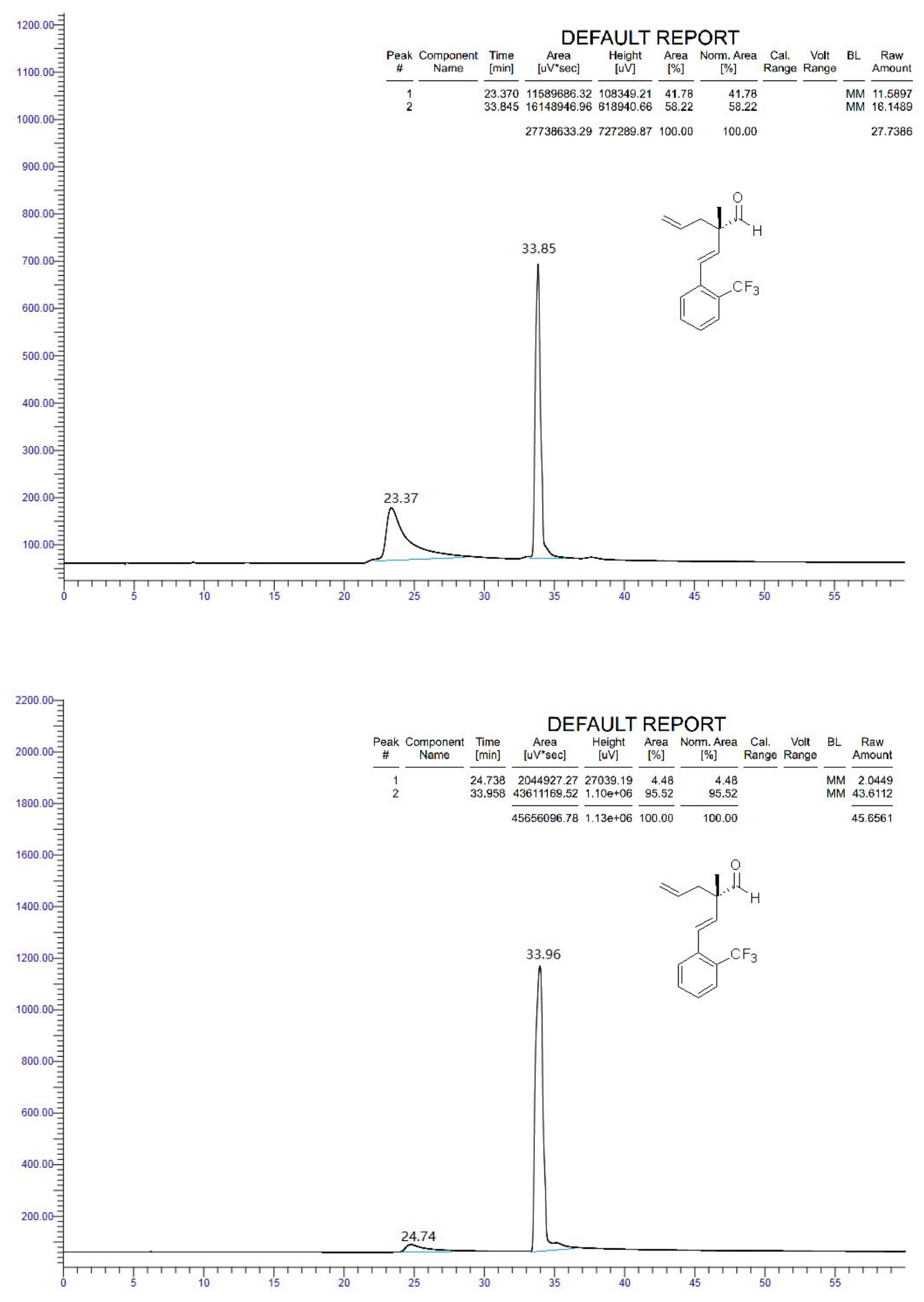


\section{2i: (S,E)-2-methyl-2-(2-(naphthalen-1-yl)vinyl)pent-4-enal}

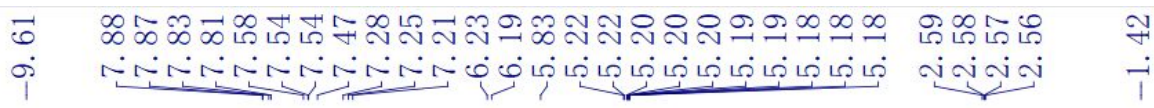

${ }^{1} \mathrm{H} \mathrm{NMR}\left(500 \mathrm{MHz}, \mathrm{CDCl}_{3}\right)$
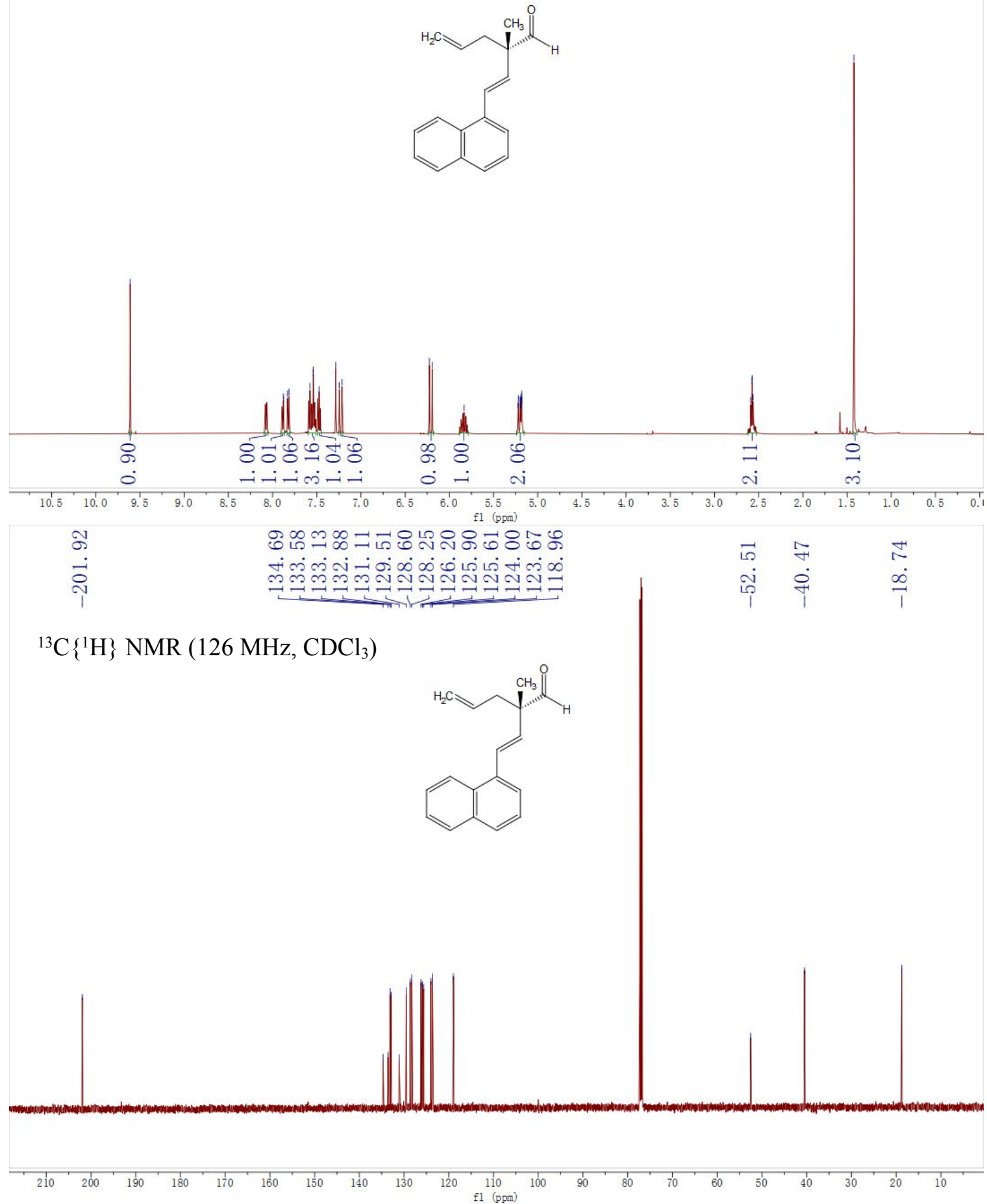

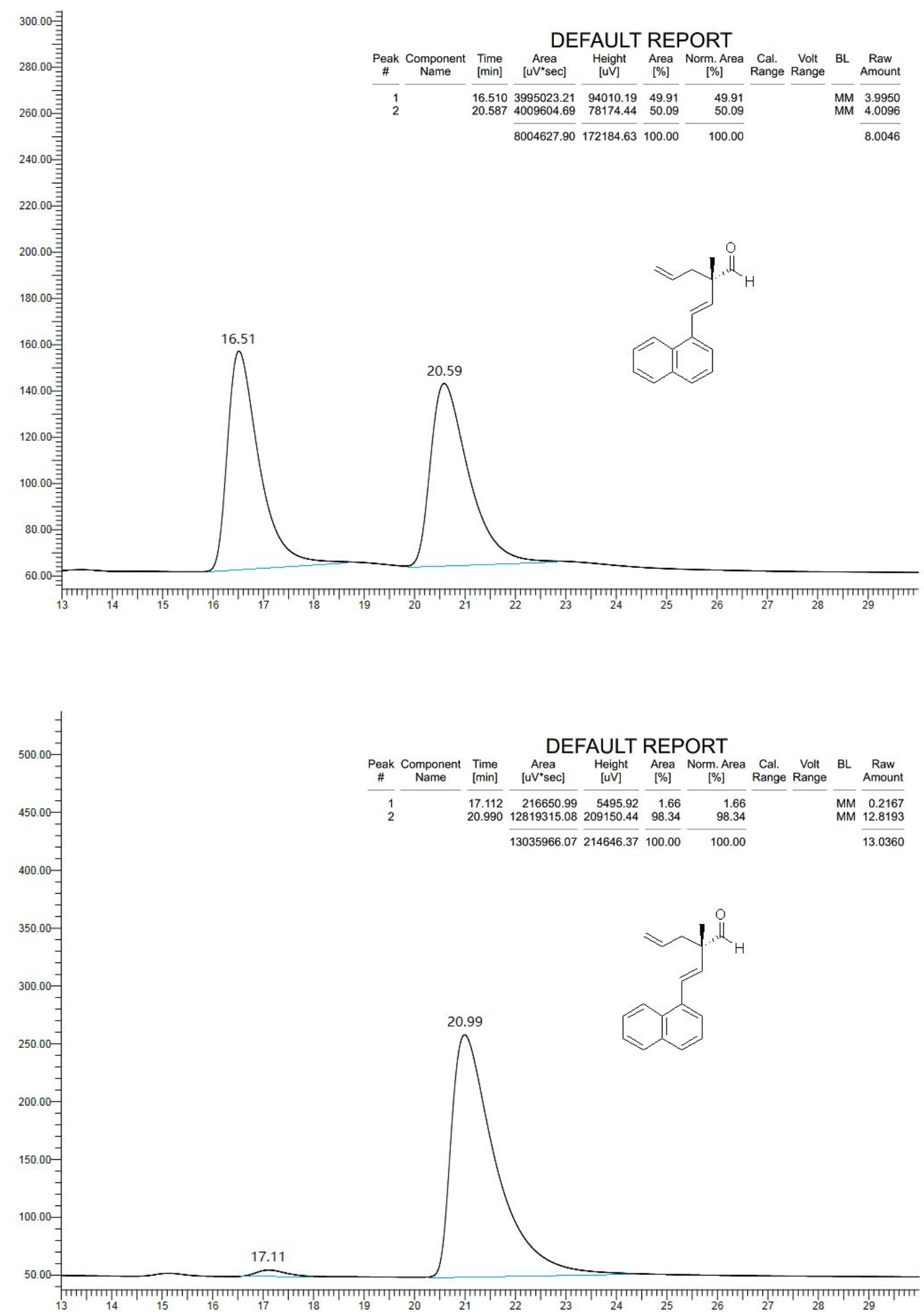


\section{2j: (R)-2-(3,4-dihydronaphthalene-2-yl)-2-methylpent-4-enal}

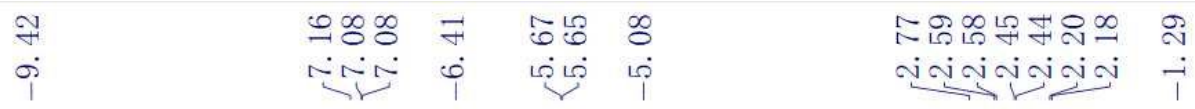

${ }^{1} \mathrm{H}$ NMR $\left(500 \mathrm{MHz}, \mathrm{CDCl}_{3}\right)$<smiles>C=CCC(C)(C=O)C1=Cc2ccccc2CC1</smiles>

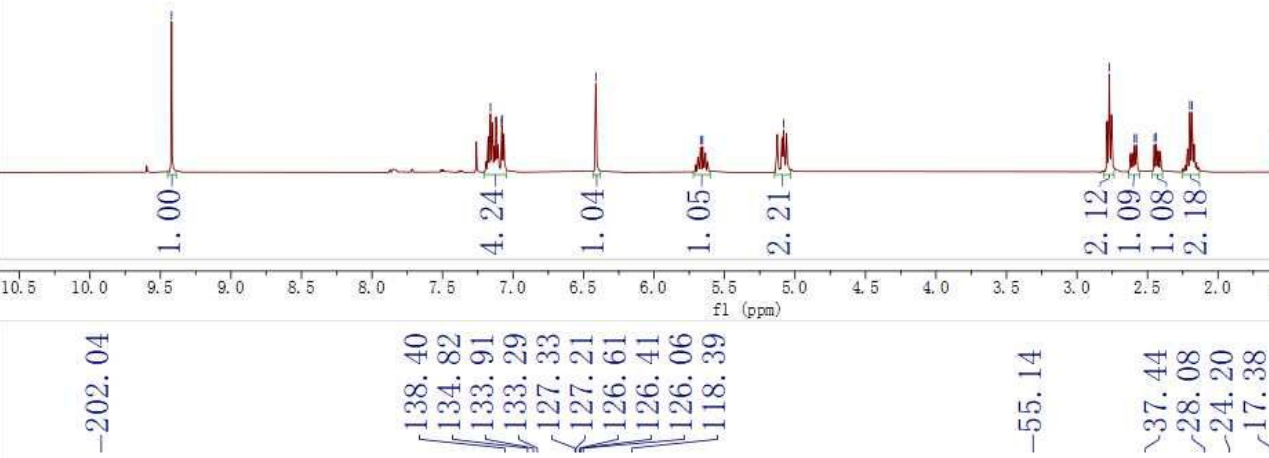

${ }^{13} \mathrm{C}\left\{{ }^{1} \mathrm{H}\right\}$ NMR $\left(126 \mathrm{MHz}, \mathrm{CDCl}_{3}\right)$<smiles>C=CCC(C)C=O</smiles>

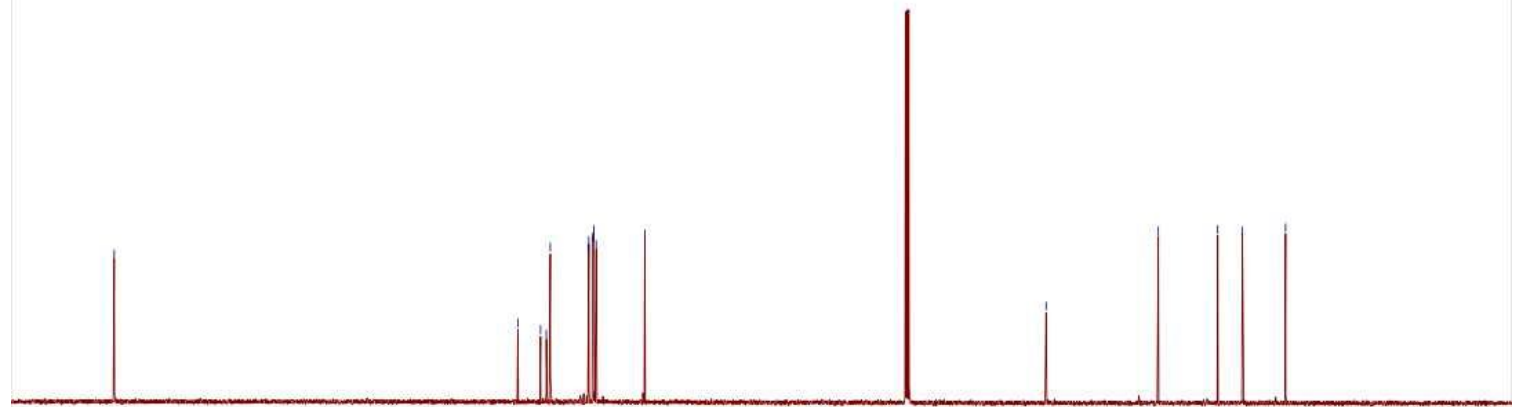

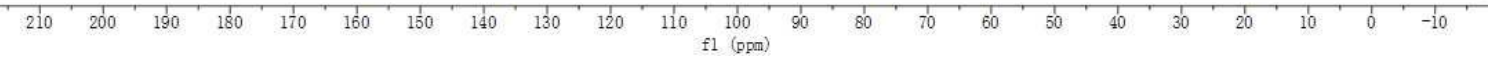




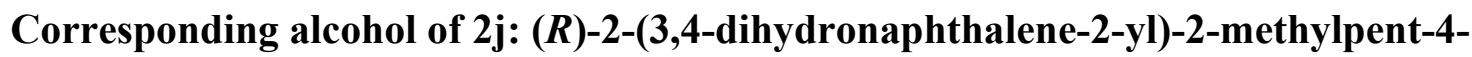
en-1-ol

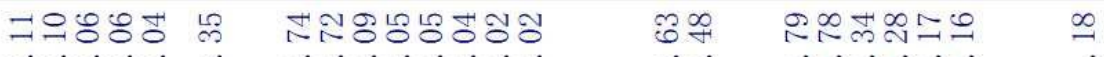

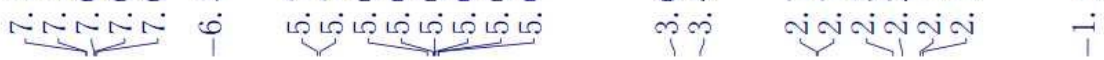

${ }^{1} \mathrm{H}$ NMR (500 MHz, $\left.\mathrm{CDCl}_{3}\right)$<smiles>C=CCC(C)(CO)C1=Cc2ccccc2CC1</smiles>

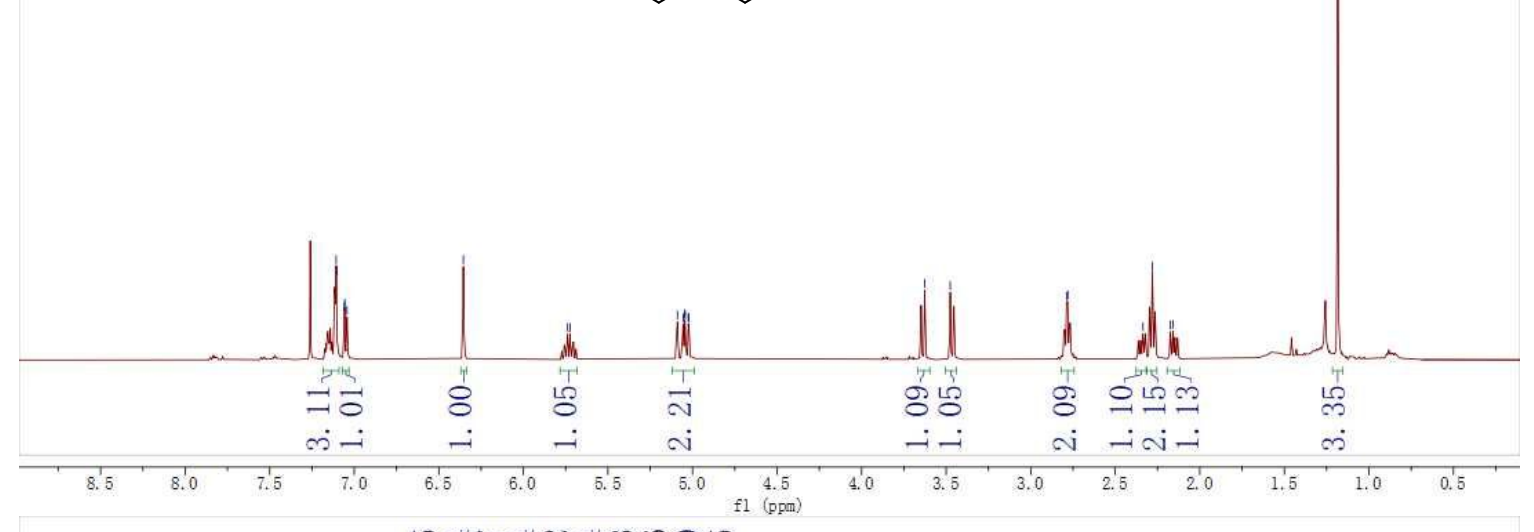

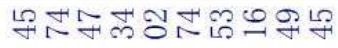

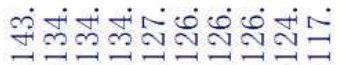

₹ $\quad$ เก

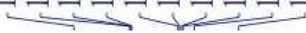

审

${ }^{13} \mathrm{C}\left\{{ }^{1} \mathrm{H}\right\} \mathrm{NMR}\left(126 \mathrm{MHz}, \mathrm{CDCl}_{3}\right)$<smiles>C=CCC(C)(CO)C1=Cc2ccccc2CC1</smiles>
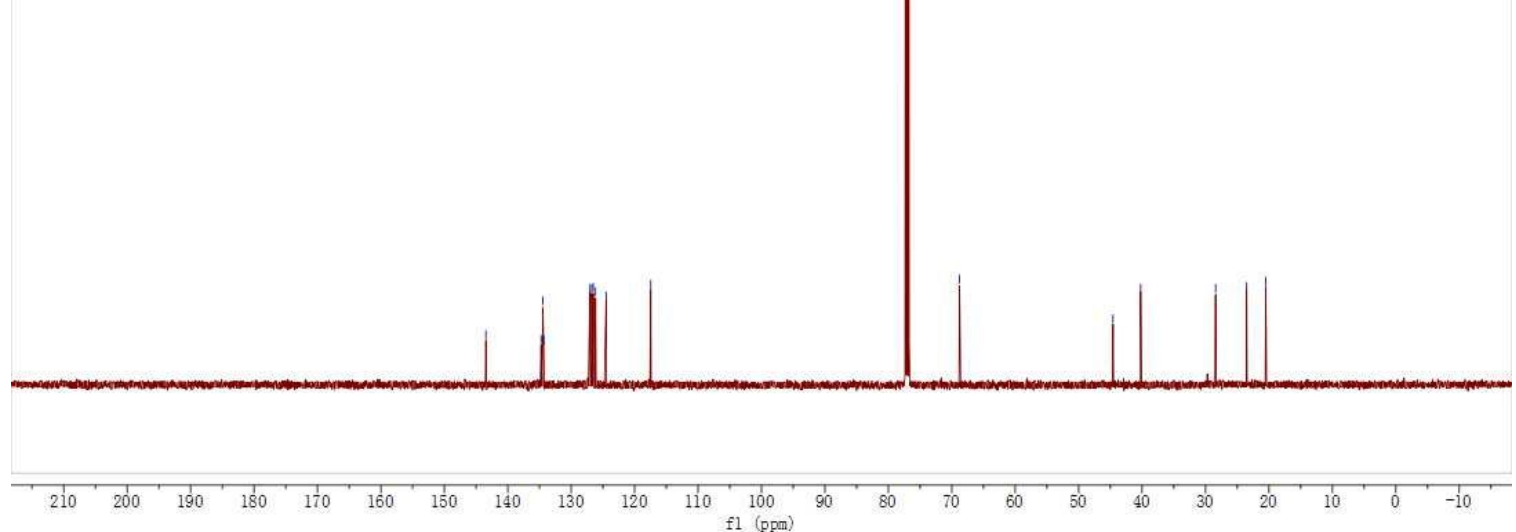

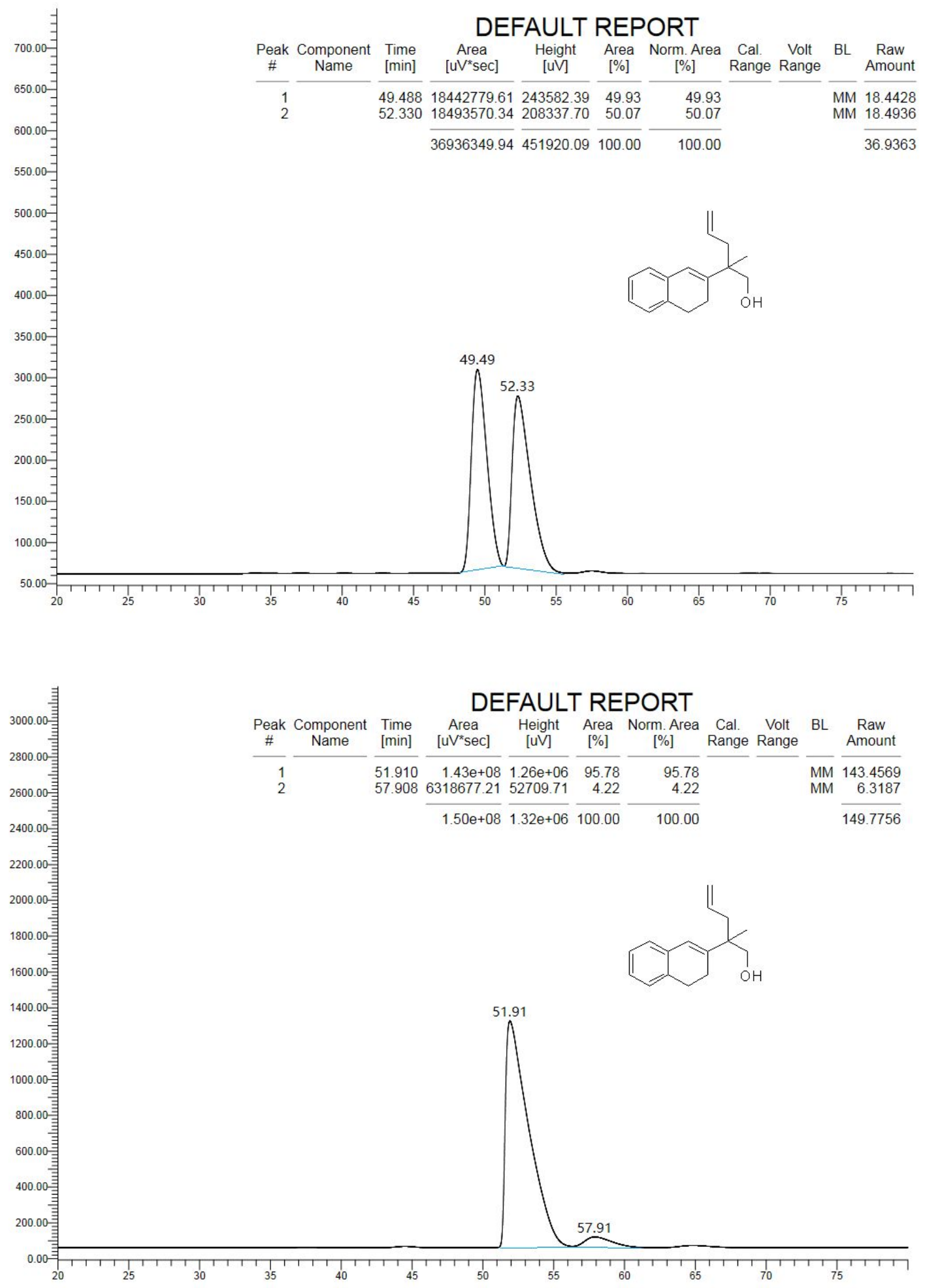
2k: tert-butyl (S,E)-2-(3-formyl-3-methylhexa-1,5-dien-1-yl)-1H-pyrrole-1carboxylate

${ }^{1} \mathrm{H} \mathrm{NMR}\left(500 \mathrm{MHz}, \mathrm{CDCl}_{3}\right)$

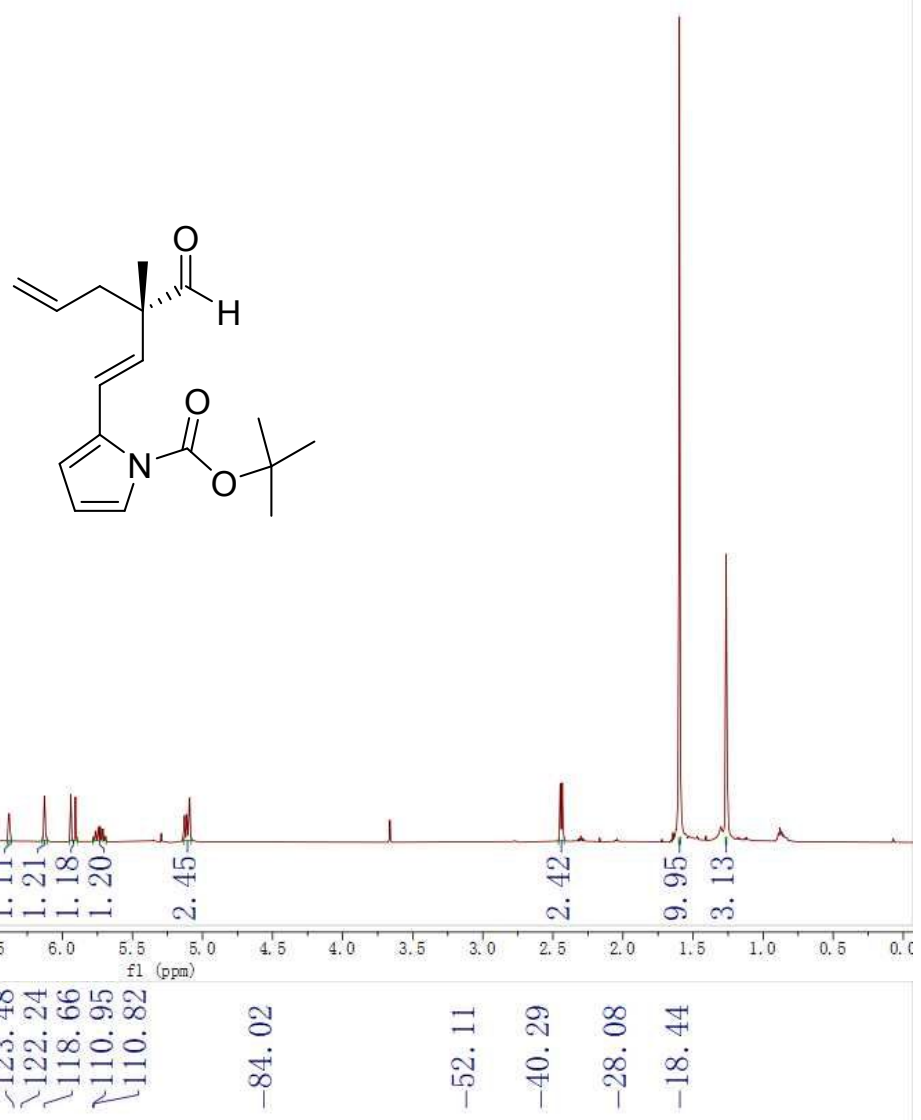

${ }^{13} \mathrm{C}\left\{{ }^{1} \mathrm{H}\right\} \mathrm{NMR}\left(126 \mathrm{MHz}, \mathrm{CDCl}_{3}\right)$
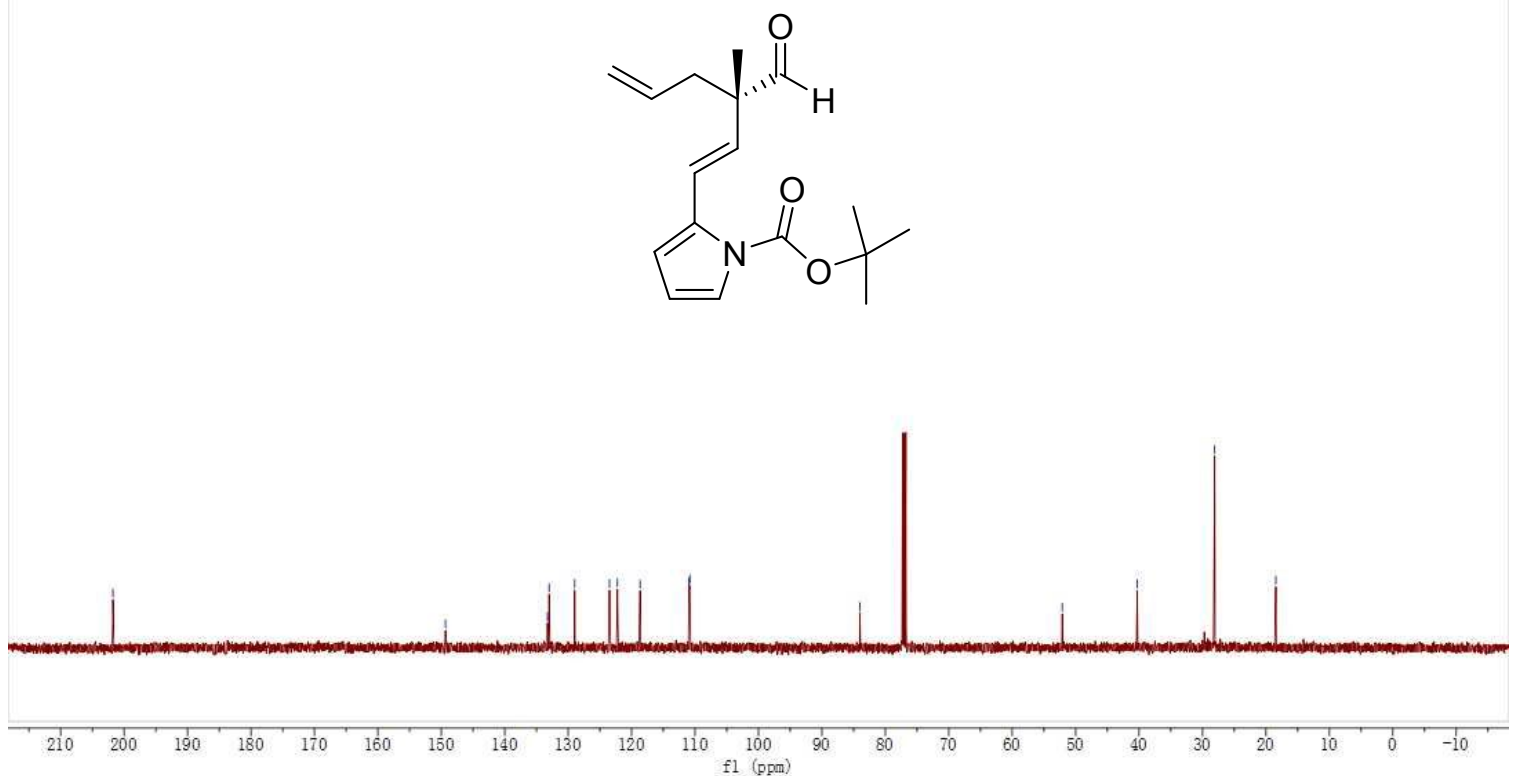

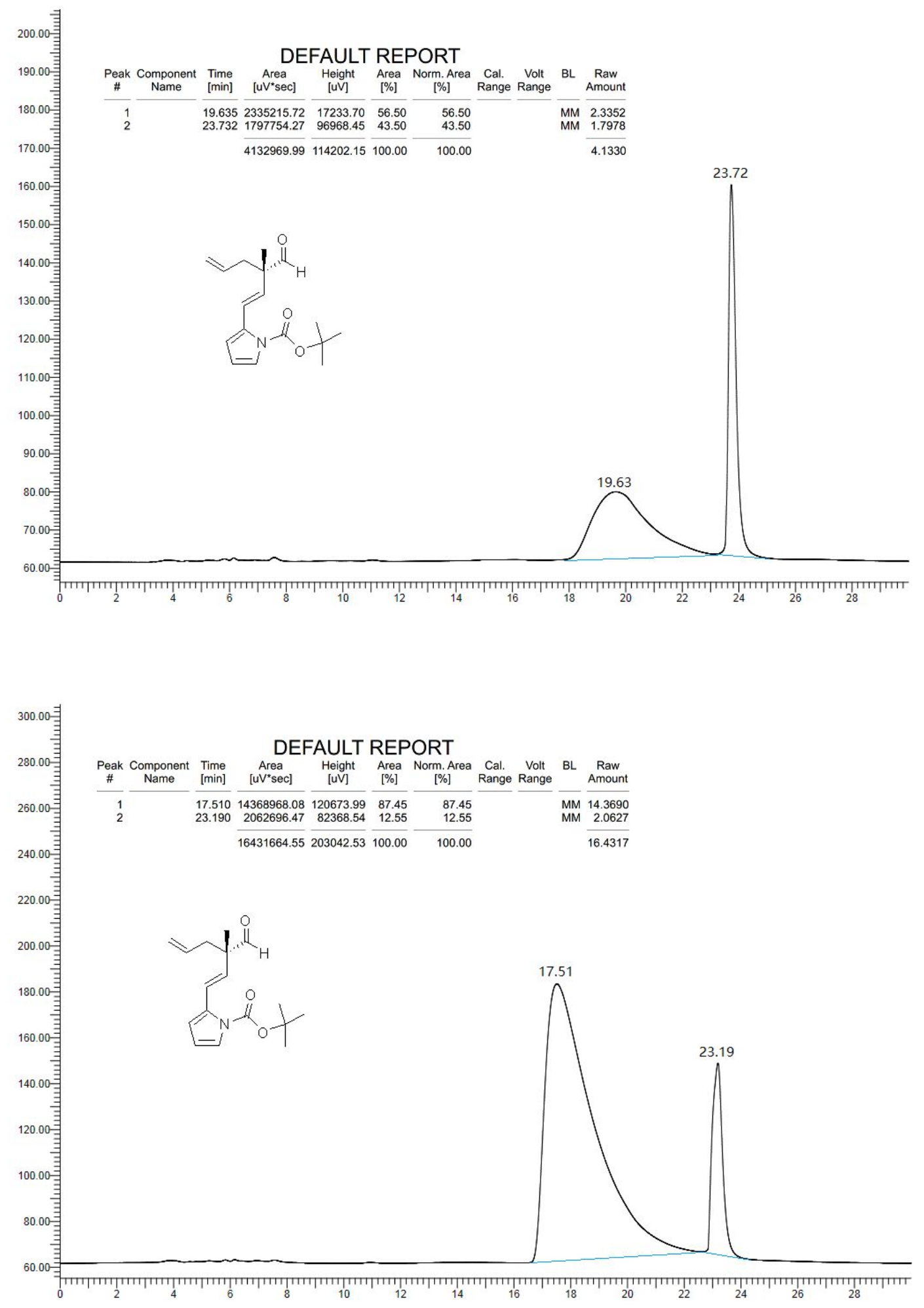


\section{I: $(S, E)$-2-ethyl-2-styrylpent-4-enal}

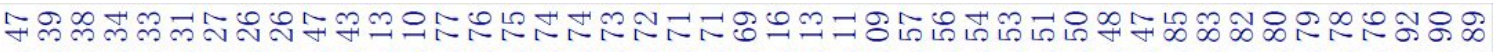
OiNAN

${ }^{1} \mathrm{H} \mathrm{NMR}\left(500 \mathrm{MHz}, \mathrm{CDCl}_{3}\right)$

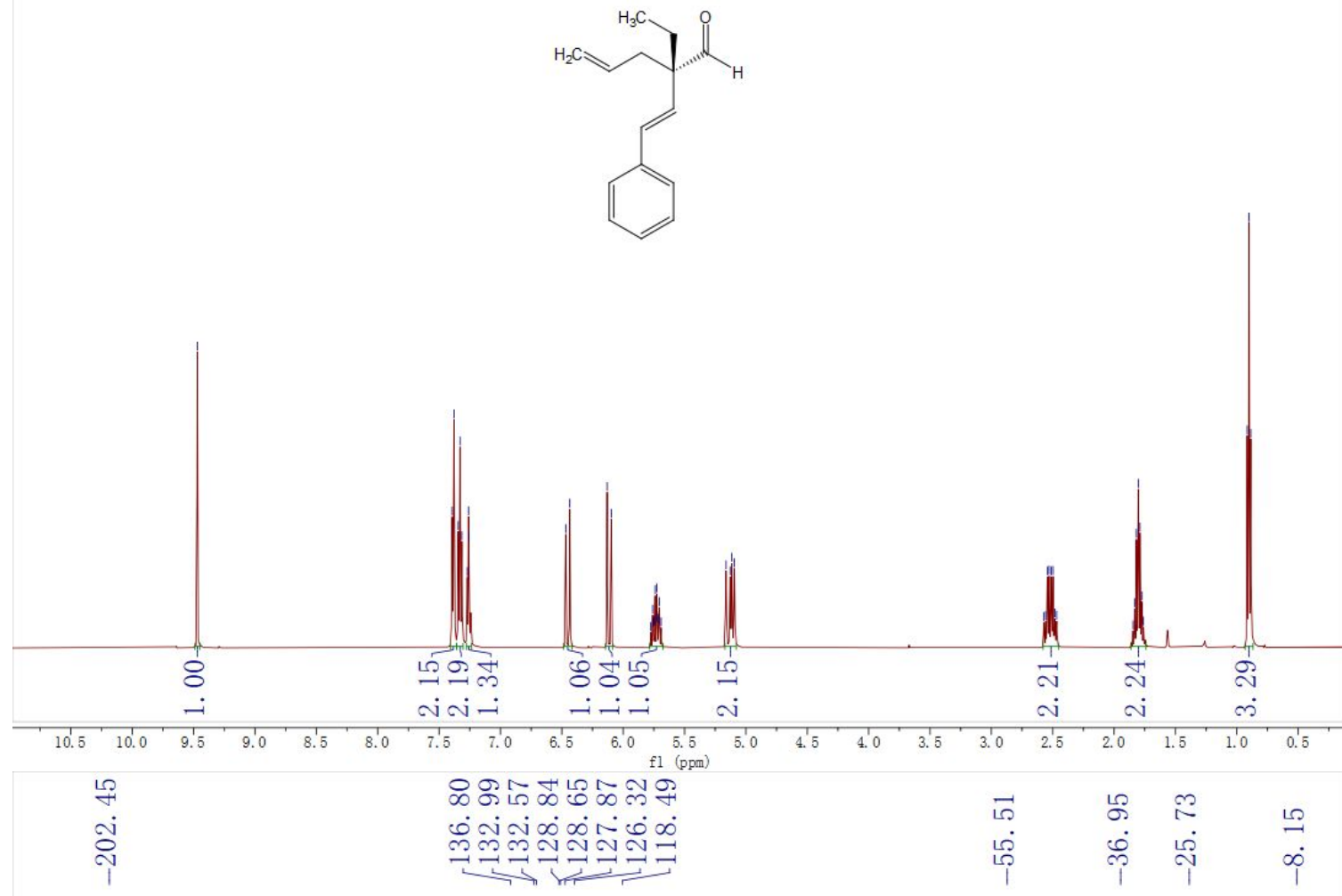

${ }^{13} \mathrm{C}\left\{{ }^{1} \mathrm{H}\right\} \mathrm{NMR}\left(126 \mathrm{MHz}, \mathrm{CDCl}_{3}\right)$

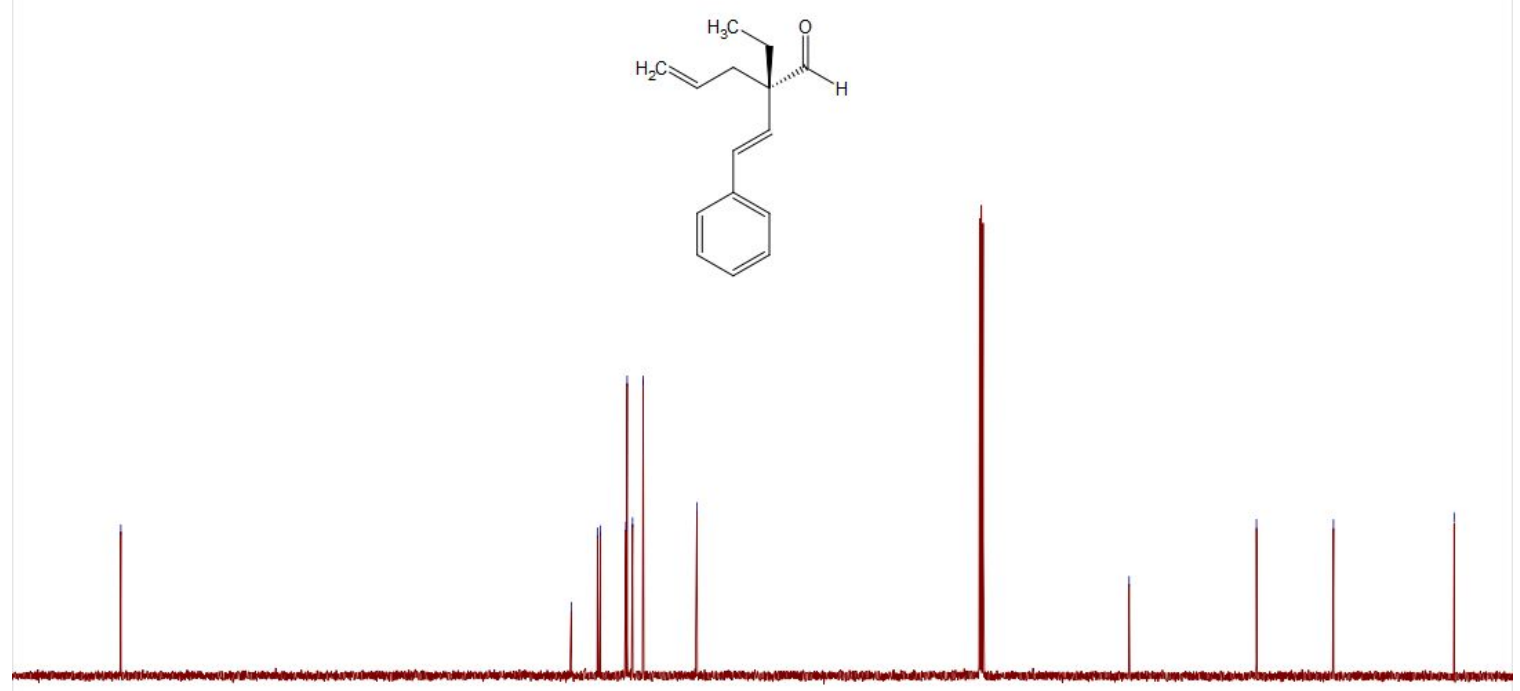

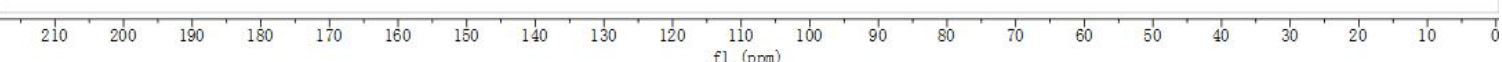




\section{Corresponding alcohol of 2l: $(S, E)$-2-ethyl-2-styrylpent-4-en-1-ol}

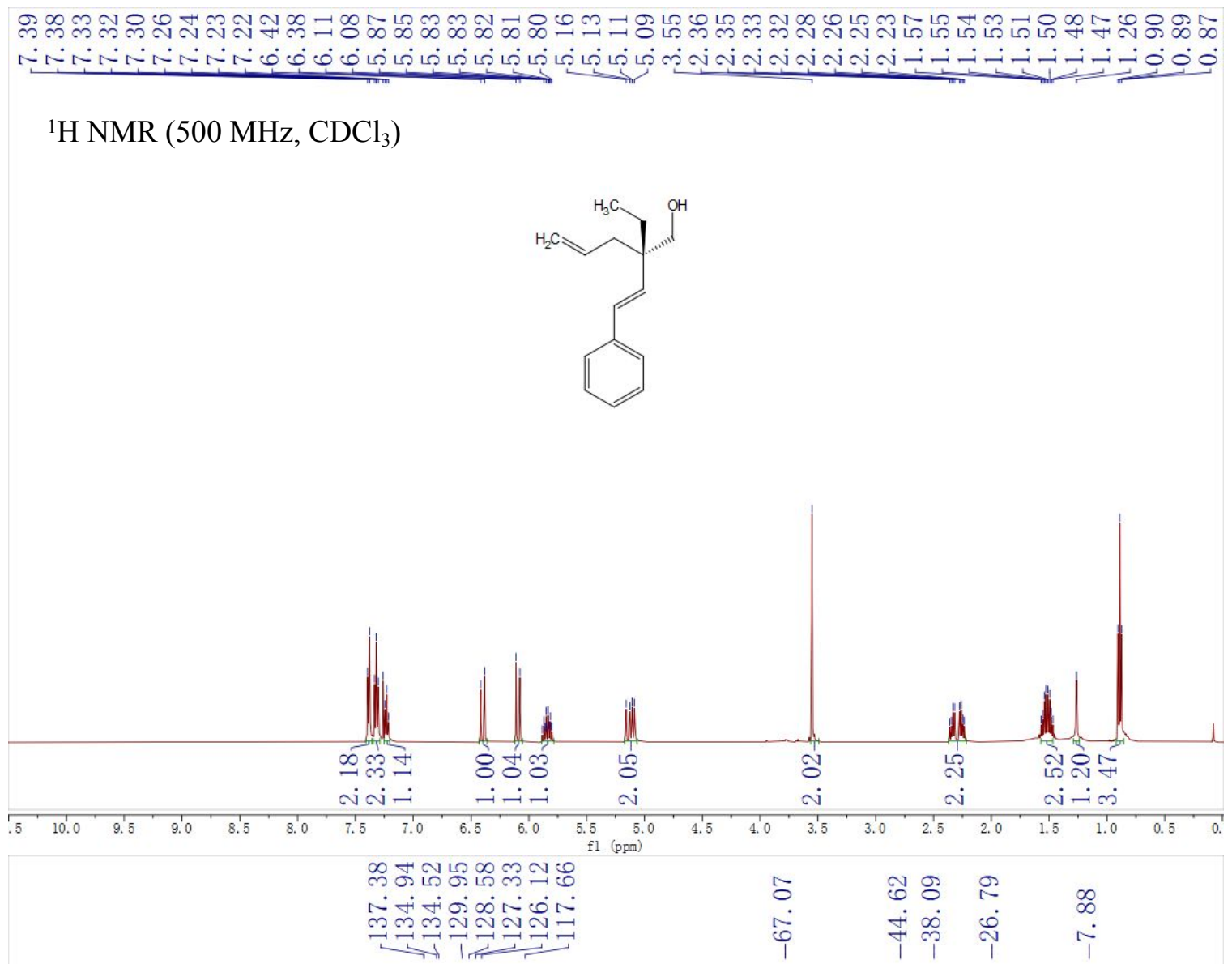

${ }^{13} \mathrm{C}\left\{{ }^{1} \mathrm{H}\right\} \mathrm{NMR}\left(126 \mathrm{MHz}, \mathrm{CDCl}_{3}\right)$
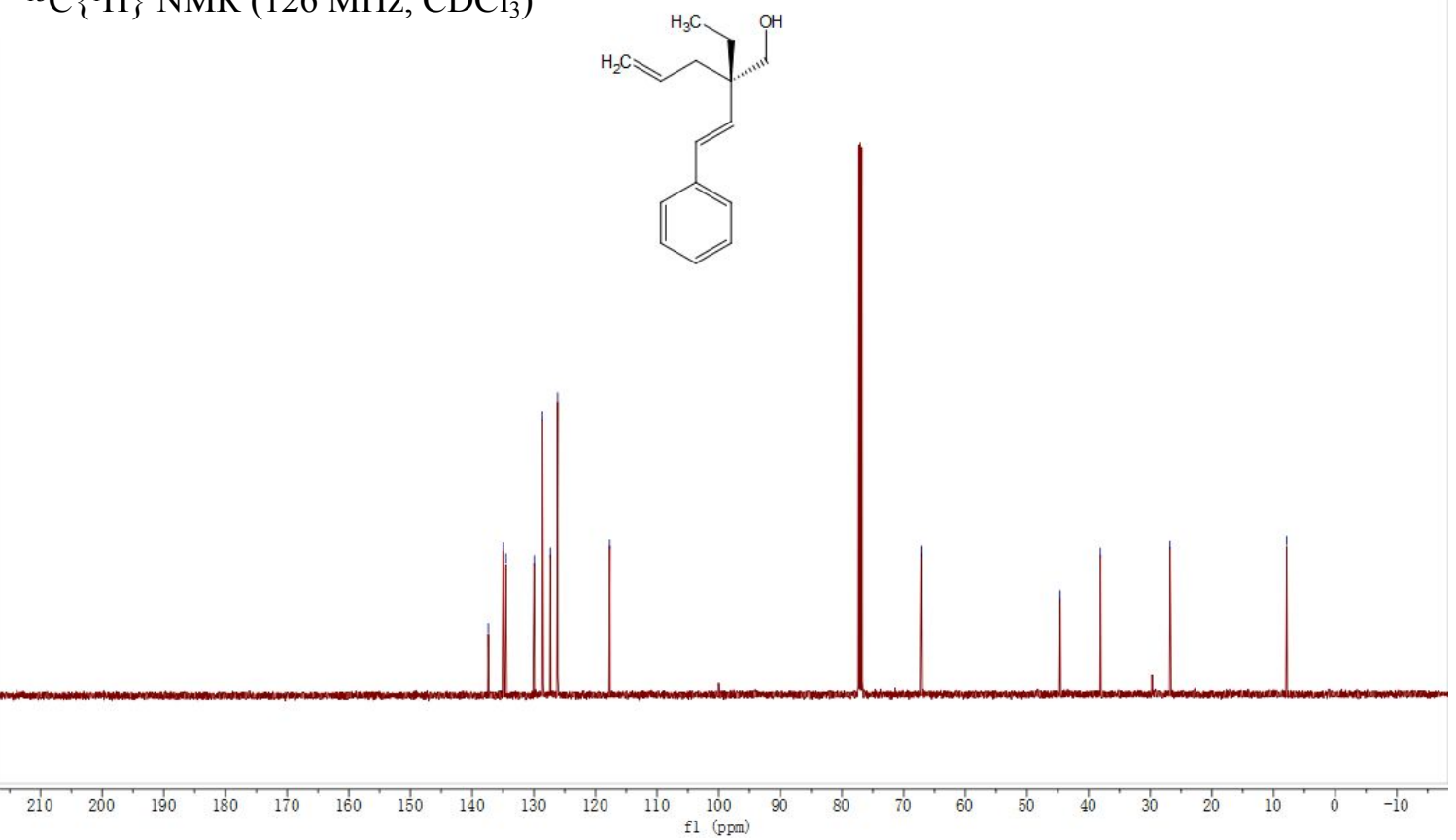


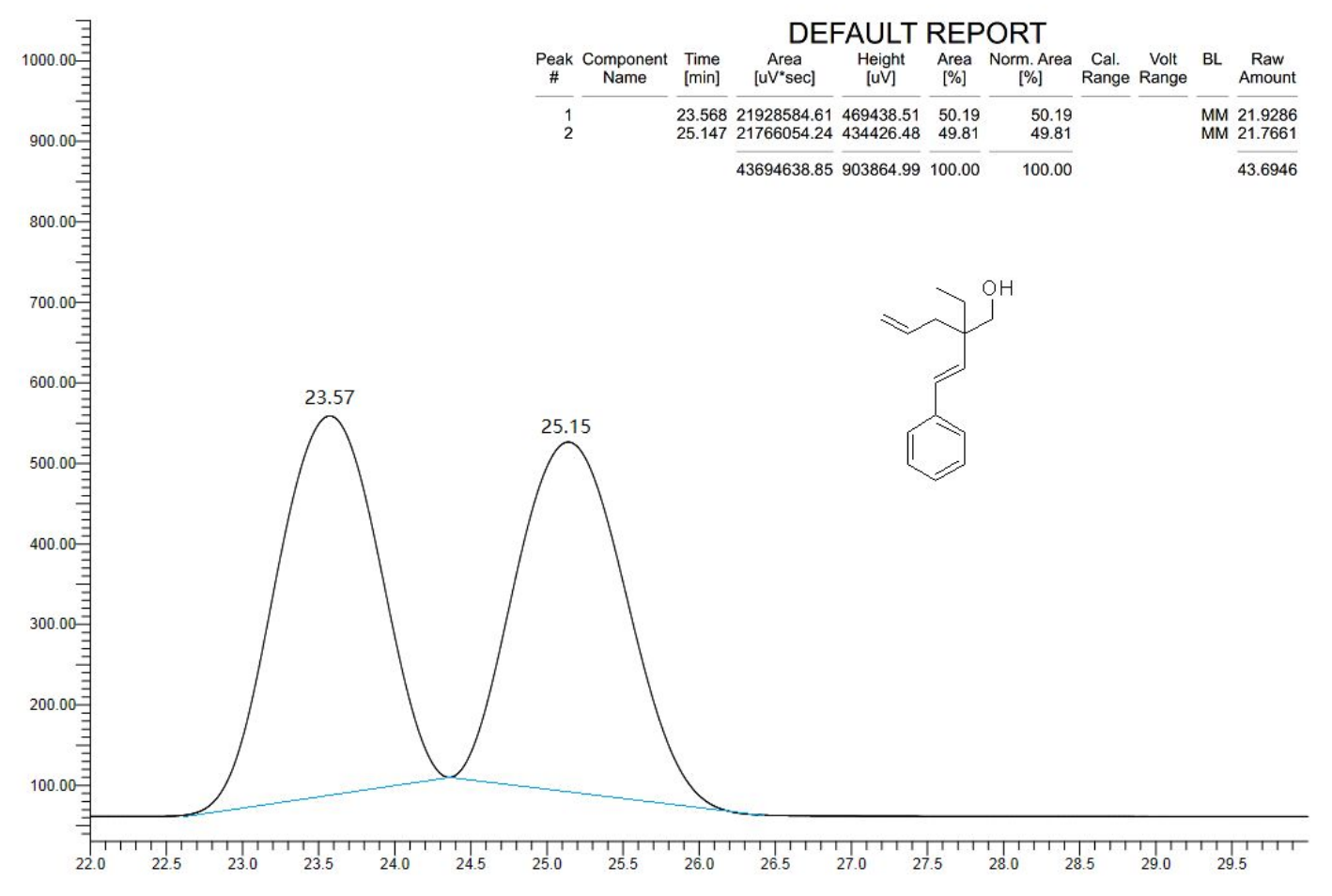

\begin{tabular}{|c|c|c|c|c|c|c|c|c|c|c|}
\hline & \multicolumn{10}{|c|}{ DEFAULT REPORT } \\
\hline $\begin{array}{c}\text { Peak } \\
\#\end{array}$ & $\begin{array}{c}\text { Component } \\
\text { Name }\end{array}$ & $\begin{array}{l}\text { Time } \\
\text { [min] }\end{array}$ & $\begin{array}{c}\text { Area } \\
{\left[\mathrm{uV}^{*} \mathrm{sec}\right]}\end{array}$ & $\begin{array}{l}\text { Height } \\
{[\mathrm{uV}]}\end{array}$ & $\begin{array}{c}\text { Area } \\
{[\%]}\end{array}$ & $\begin{array}{c}\text { Norm. Area } \\
{[\%]}\end{array}$ & $\begin{array}{c}\text { Cal. } \\
\text { Range }\end{array}$ & $\begin{array}{c}\text { Volt } \\
\text { Range }\end{array}$ & $\mathrm{BL}$ & $\begin{array}{c}\text { Raw } \\
\text { Amount }\end{array}$ \\
\hline \multirow{3}{*}{$\begin{array}{l}1 \\
2\end{array}$} & & 23.025 & 63053075.32 & $2.40 \mathrm{e}+06$ & 89.86 & 89.86 & & & MM & 63.0531 \\
\hline & & 24.610 & 7116147.89 & 183596.51 & 10.14 & 10.14 & & & $\mathrm{MM}$ & 7.1161 \\
\hline & & & 70169223.21 & $2.59 \mathrm{e}+06$ & 100.00 & 100.00 & & & & 70.1692 \\
\hline
\end{tabular}

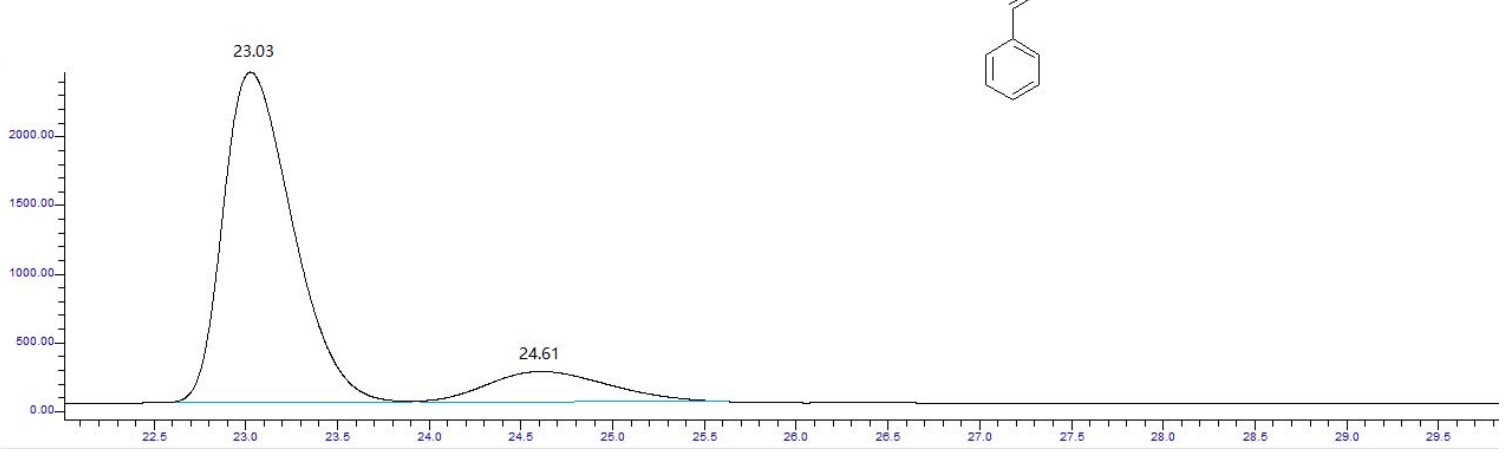




\section{3b: $(S, E)-2-m e t h y l-5-p h e n y l-2-((E)-s t y r y l) p e n t-4-e n a l$}
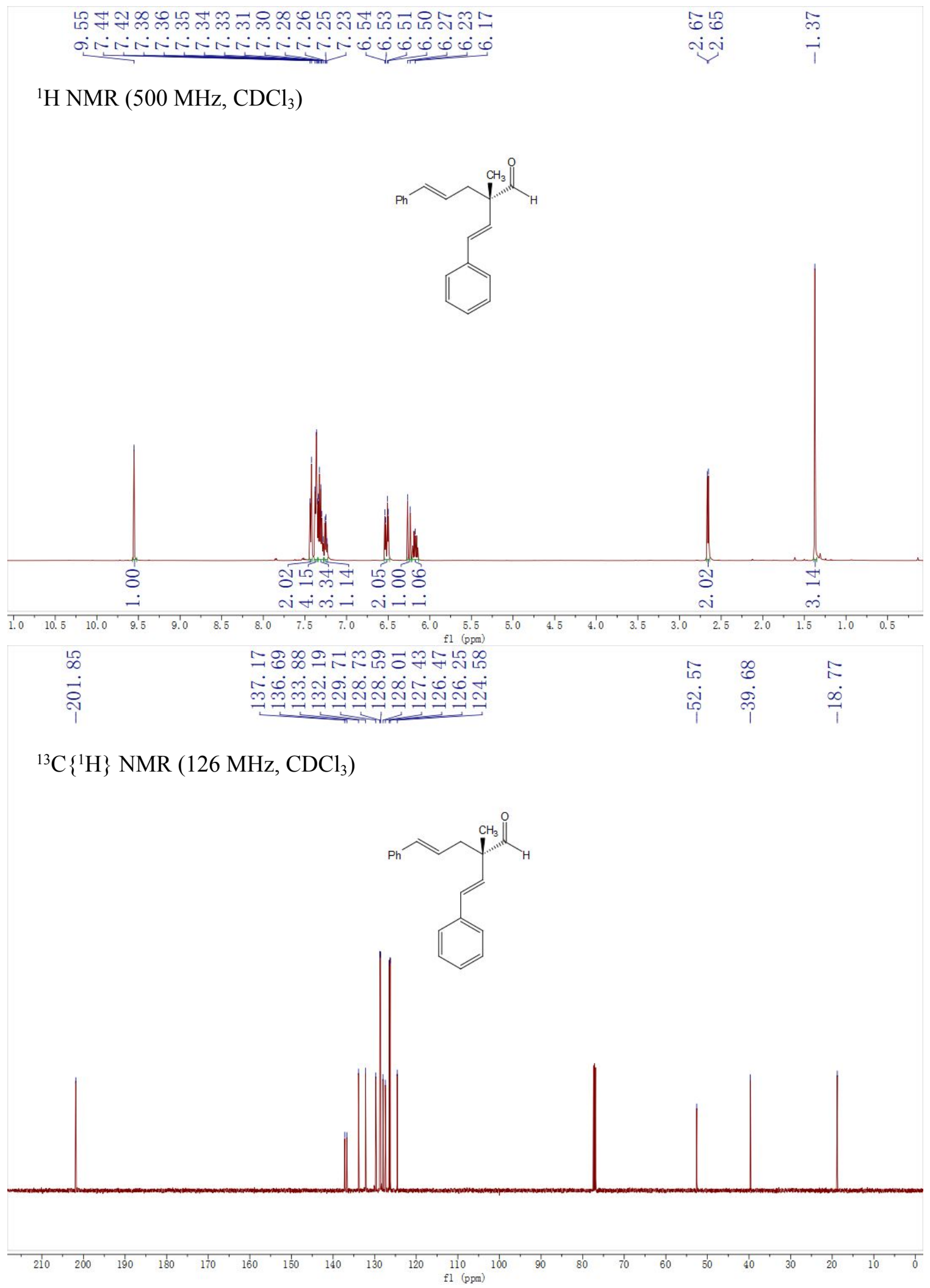

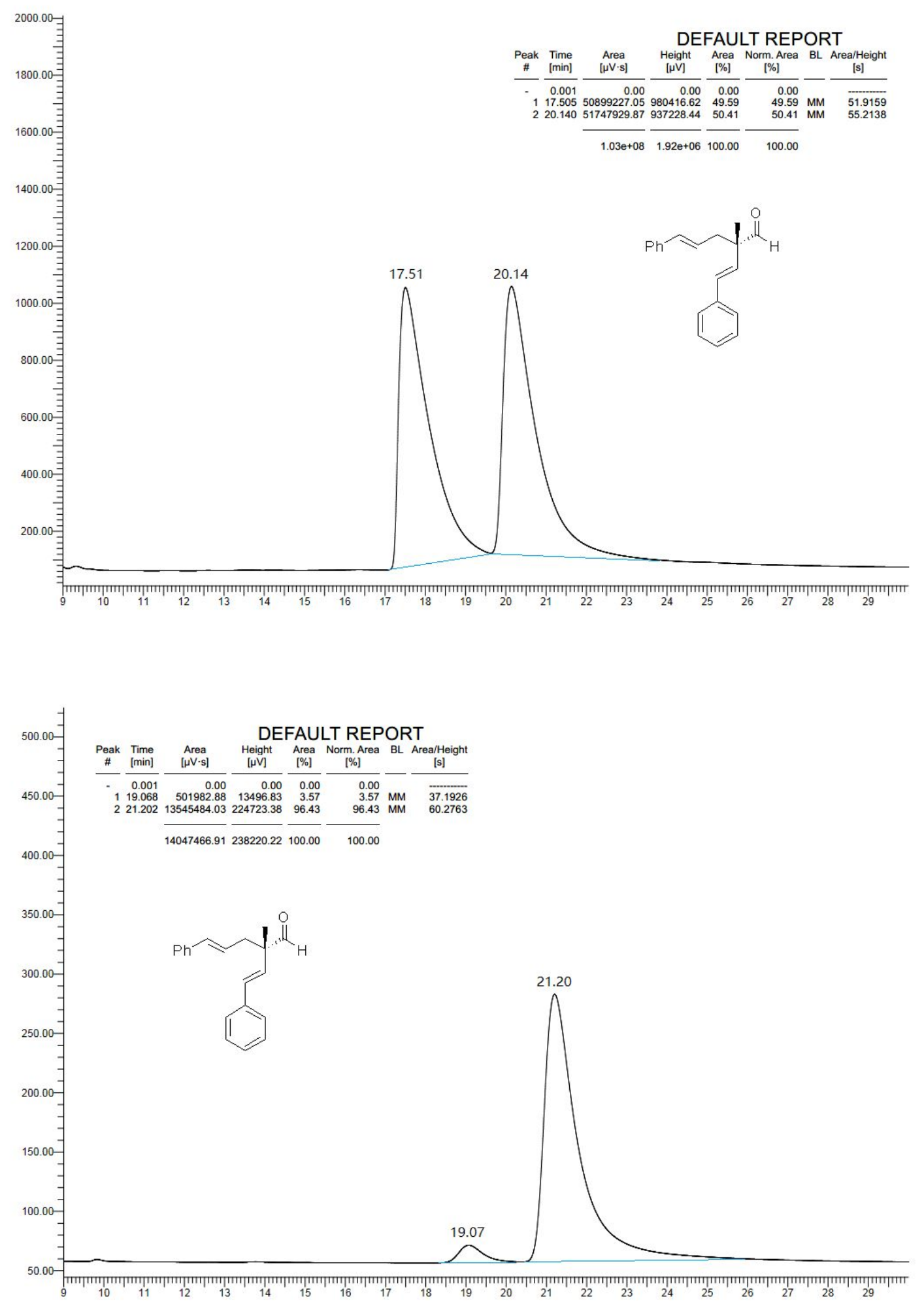
3a: $(S, E)-2,4-d i m e t h y l-2-s t y r y l p e n t-4-e n a l$
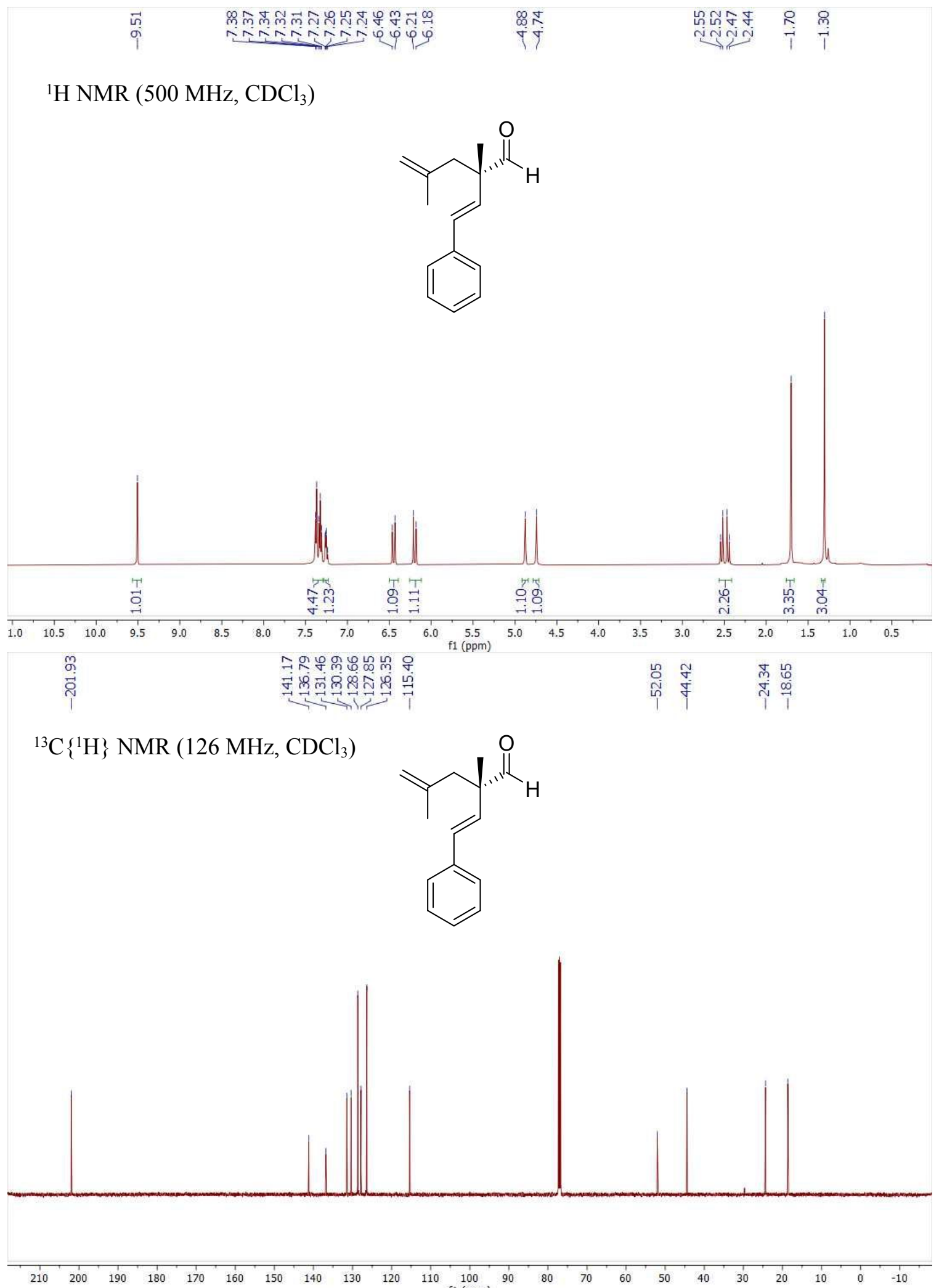

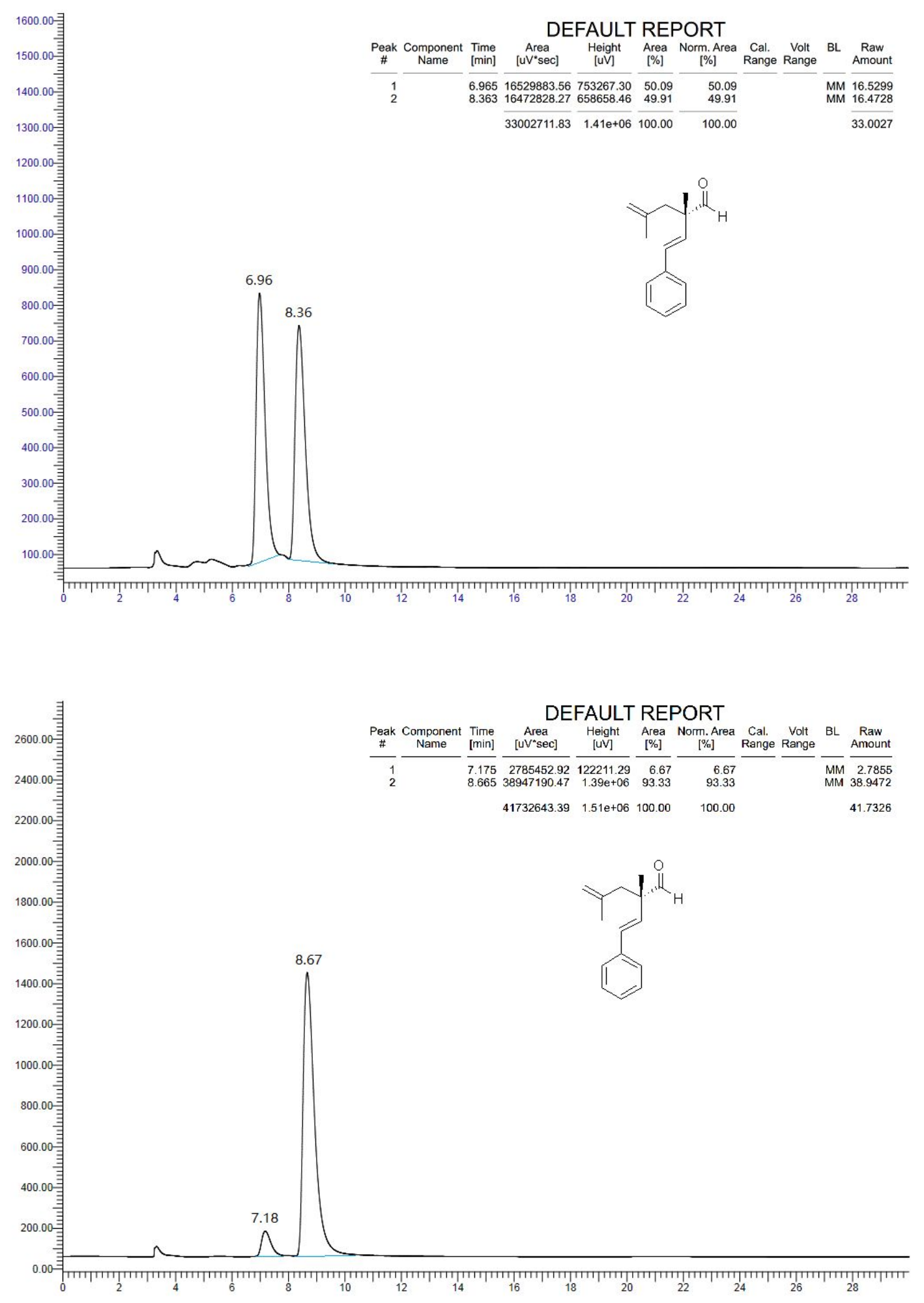
3a-y: (E)-2,6-dimethyl-4-phenylhepta-2,6-dienal

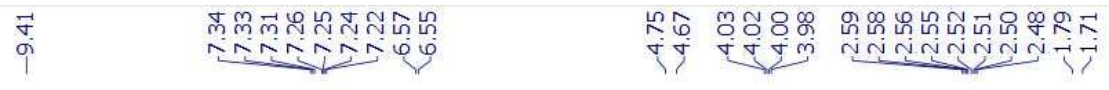

${ }^{1} \mathrm{H}$ NMR (500 MHz, $\left.\mathrm{CDCl}_{3}\right)$<smiles>C=C(C)CC(/C=C/C(C)=O)c1ccccc1</smiles>

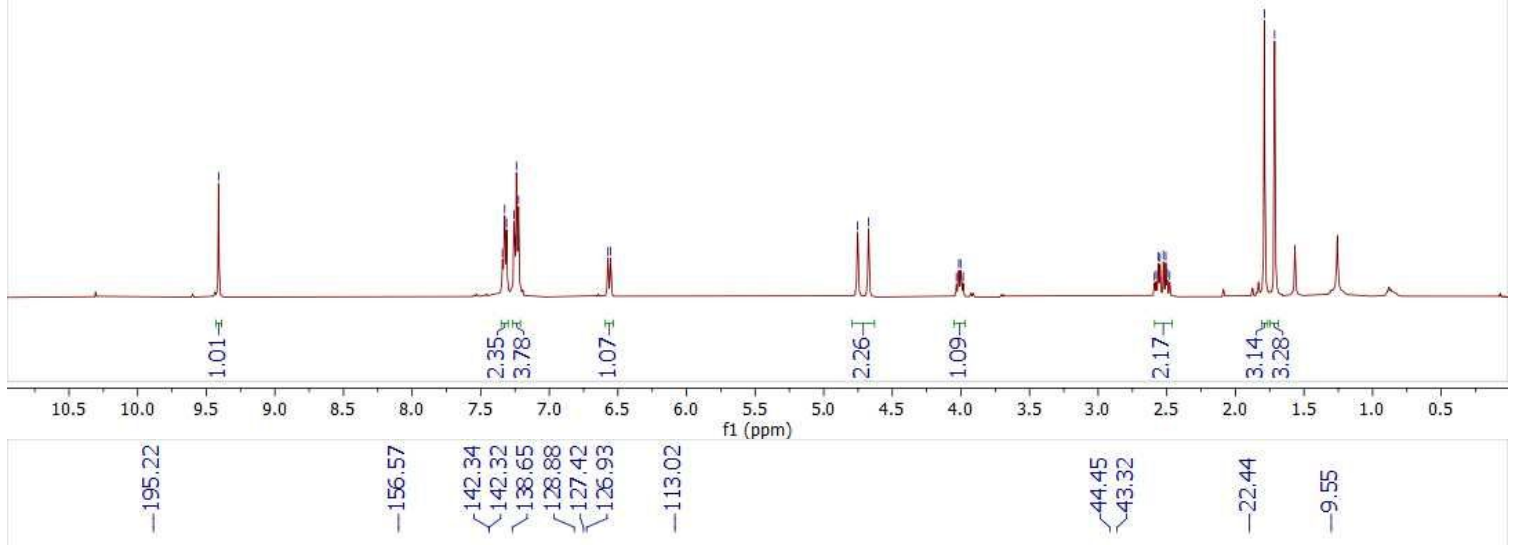

${ }^{13} \mathrm{C}\left\{{ }^{1} \mathrm{H}\right\}$ NMR $\left(126 \mathrm{MHz}, \mathrm{CDCl}_{3}\right)$<smiles>C=C(C)CC(/C=C/C(C)=O)c1ccccc1</smiles>
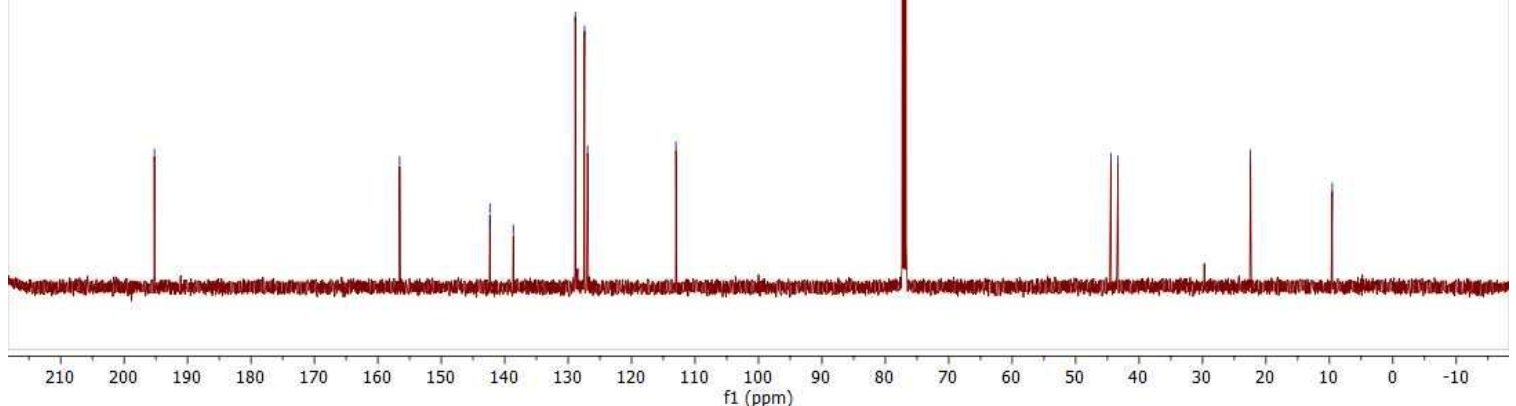

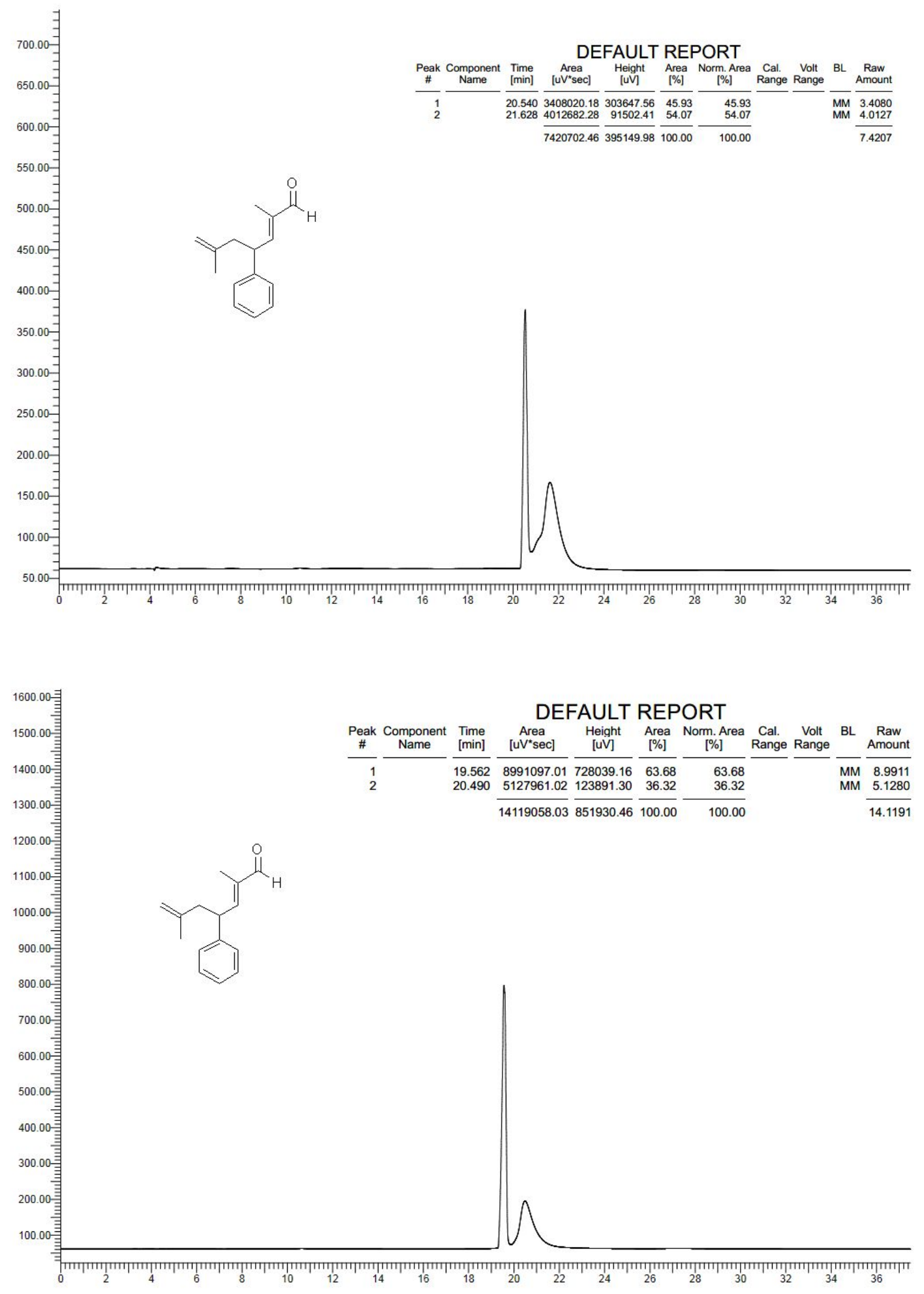
6: (E)-1-methoxy-4-(3-methyl-3-vinylhexa-1,5-dien-1-yl)benzene:
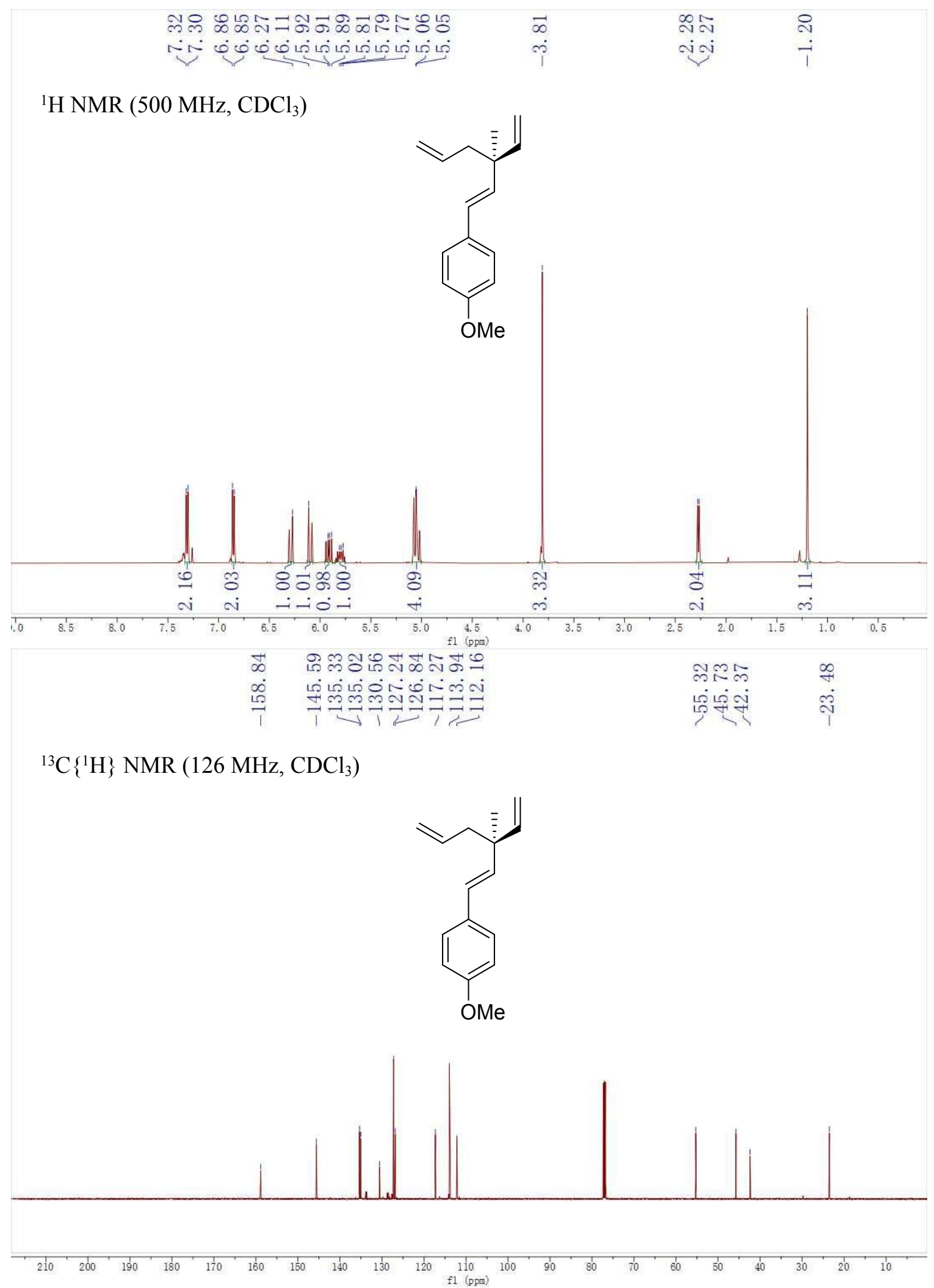
(S,E)-6-(4-methoxyphenyl)-4-methyl-4-vinylhex-5-en-1-ol:
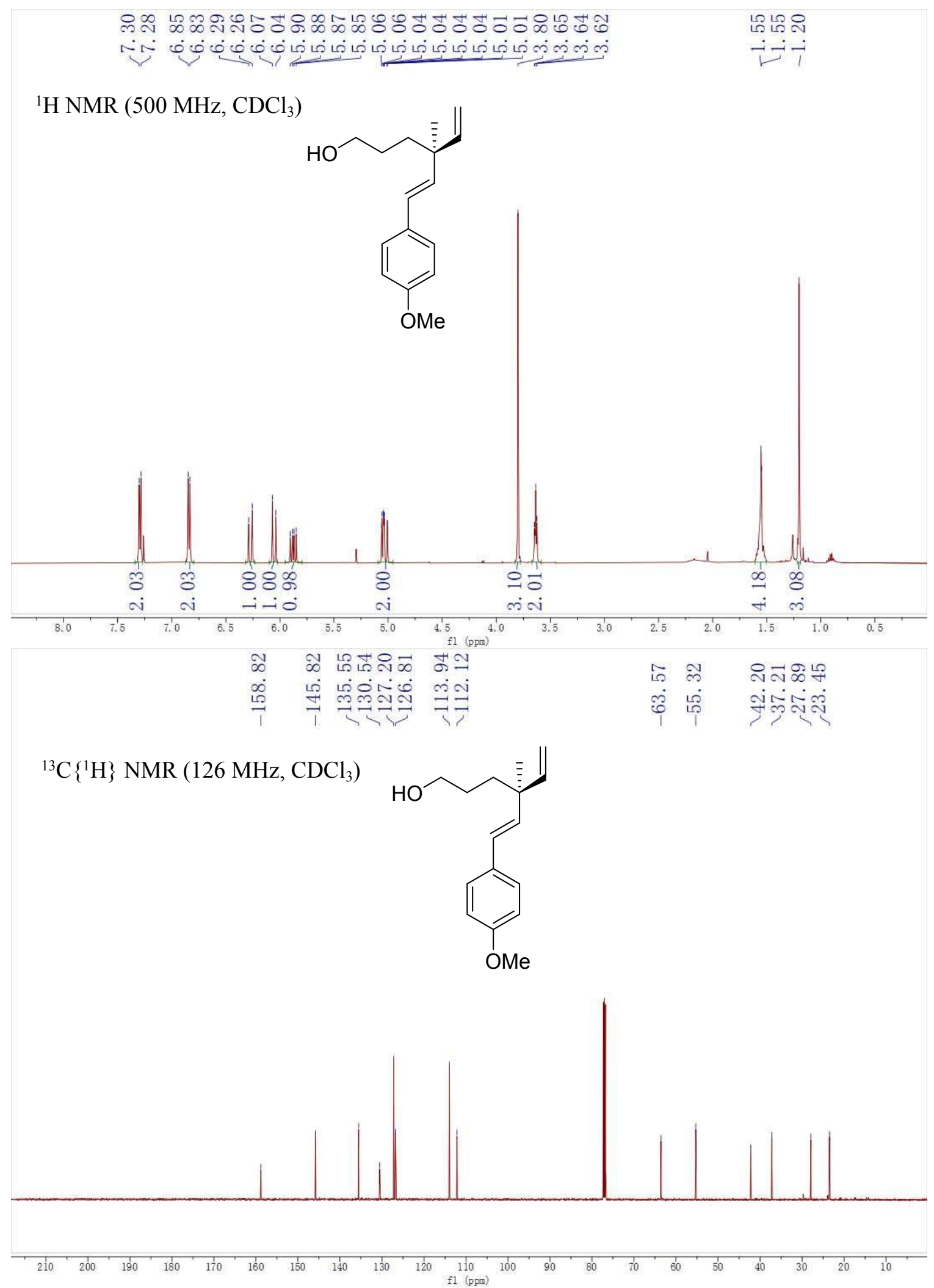


\section{7: (S,E)-6-(4-methoxyphenyl)-4-methyl-4-vinylhex-5-enal:}
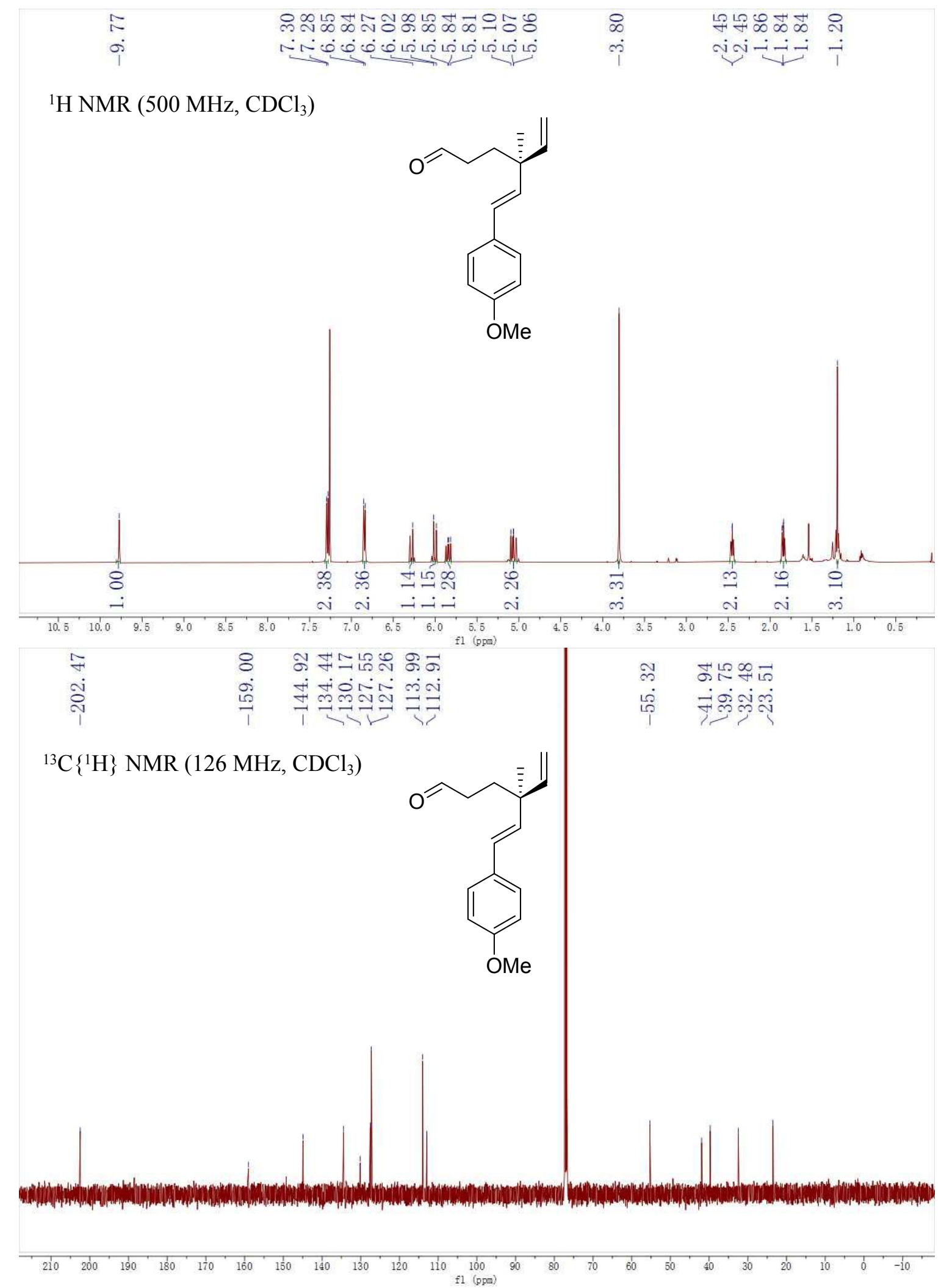


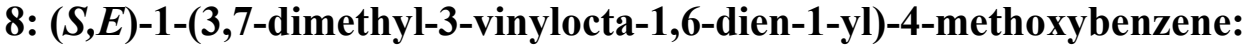

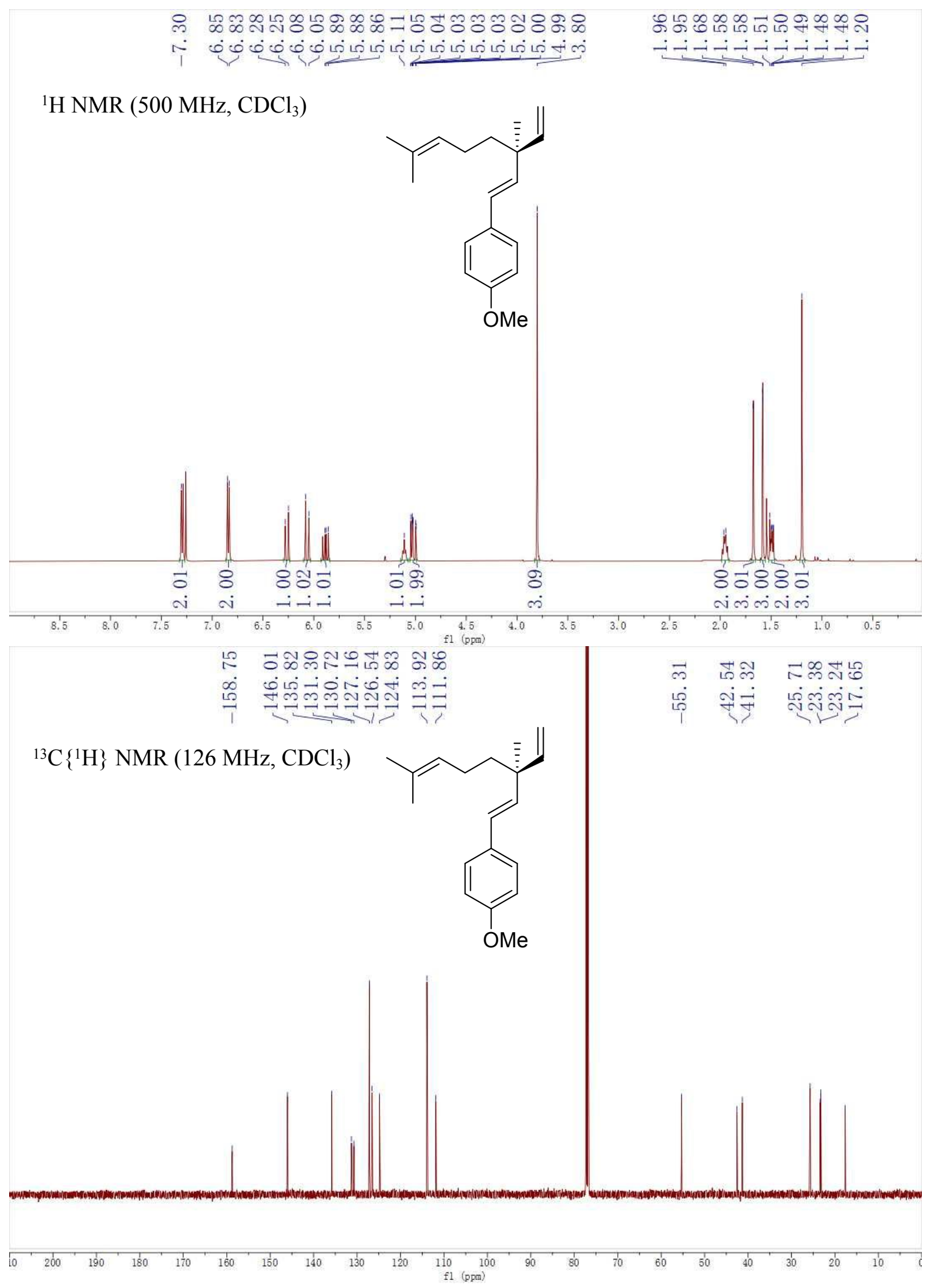




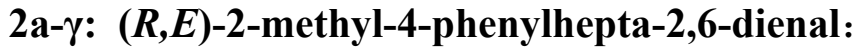

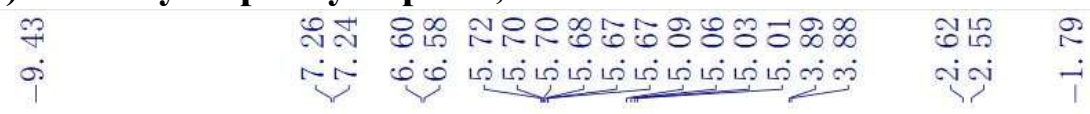

${ }^{1} \mathrm{H}$ NMR $\left(500 \mathrm{MHz}, \mathrm{CDCl}_{3}\right)$
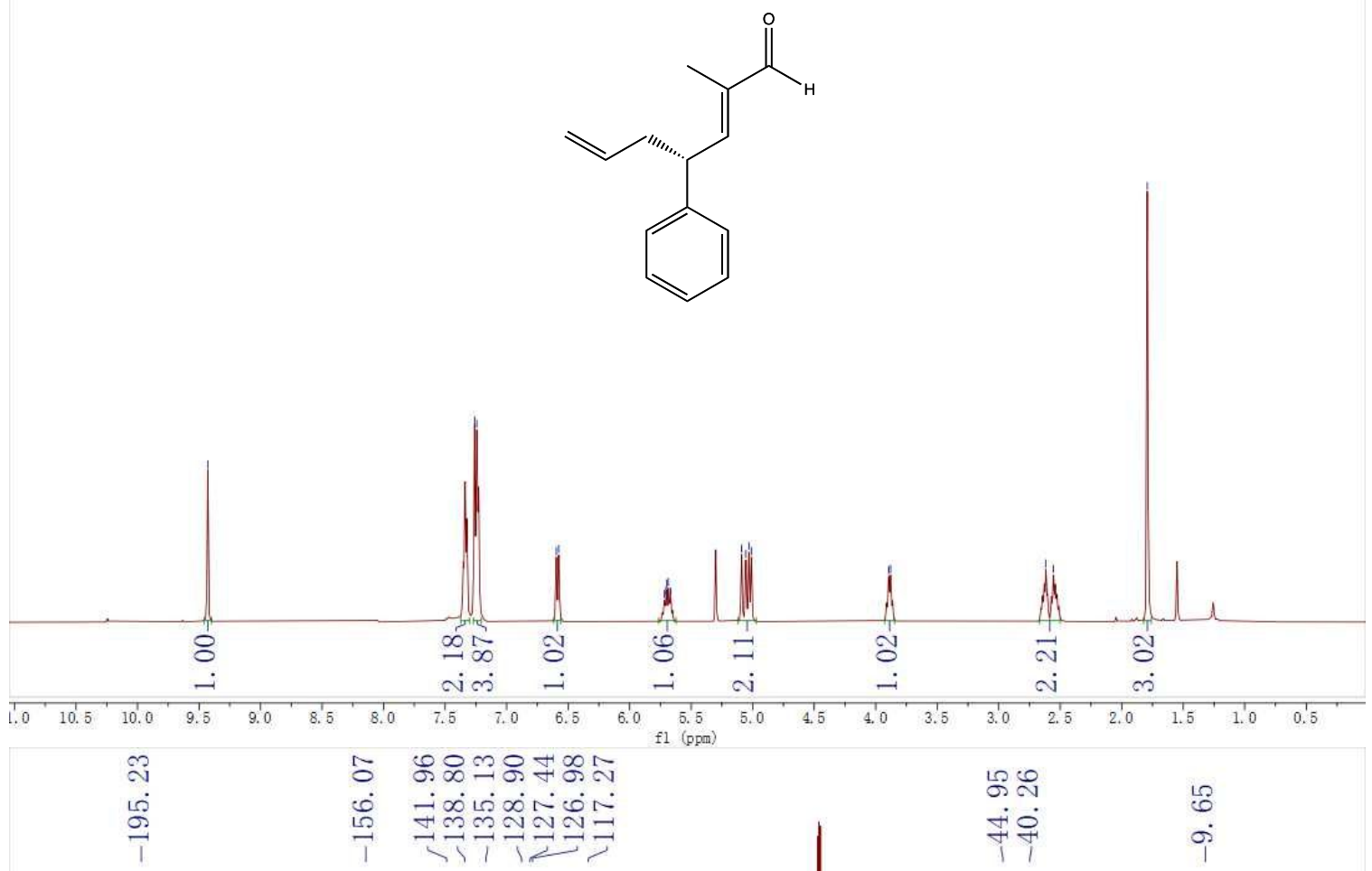

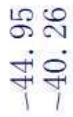

8

${ }^{13} \mathrm{C}\left\{{ }^{1} \mathrm{H}\right\}$ NMR (126 MHz, $\left.\mathrm{CDCl}_{3}\right)$<smiles>C=CC[C@H](/C=C(\C)C=O)c1ccccc1</smiles>
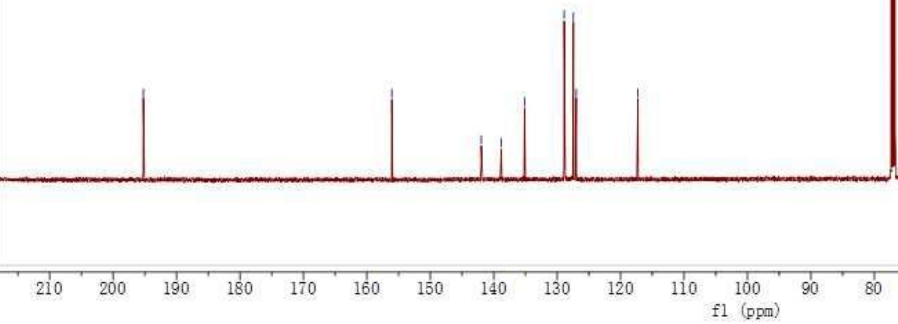

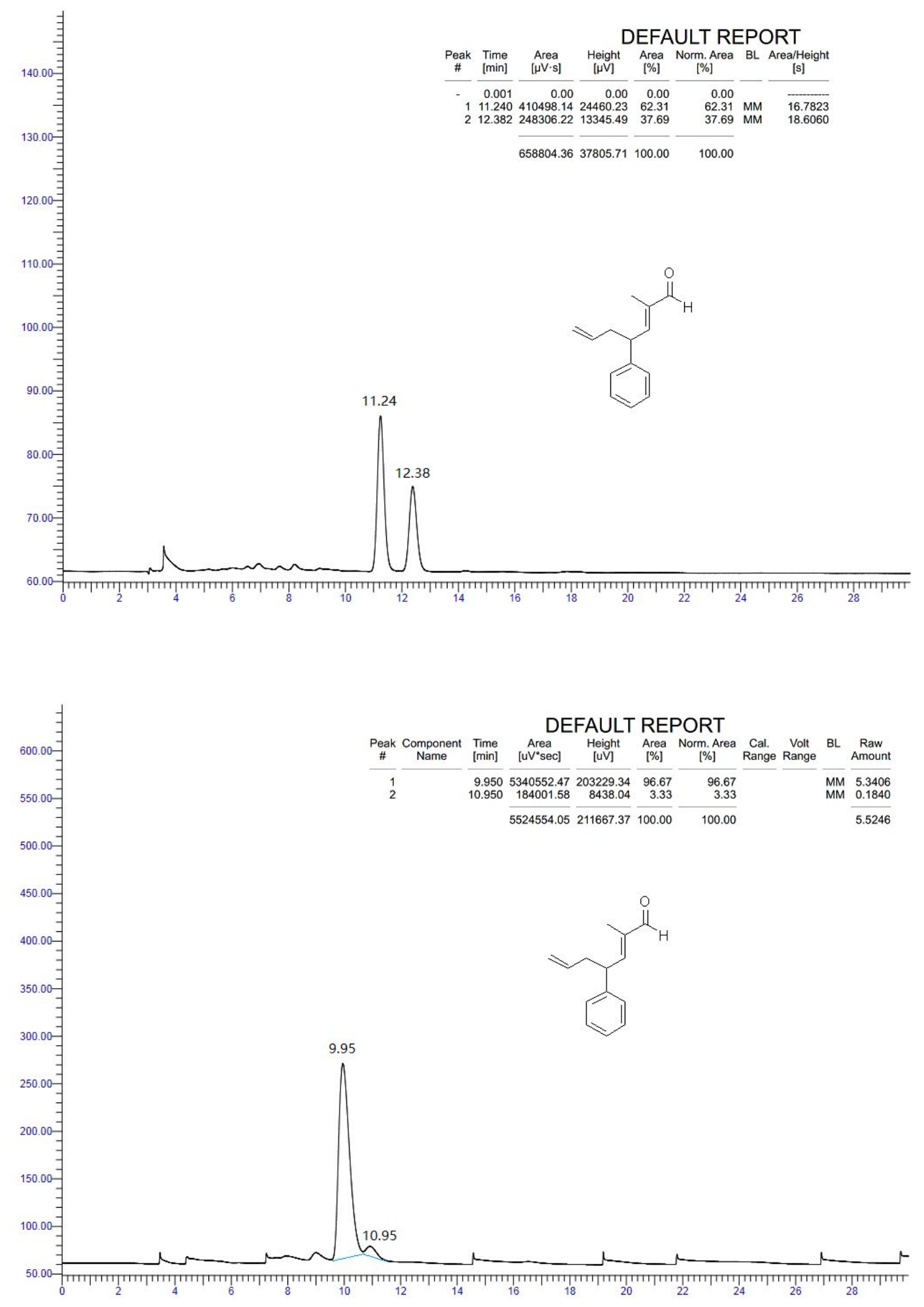